\title{
TOWARDS THE ANDRE-OORT CONJECTURE FOR MIXED SHIMURA VARIETIES: THE AX-LINDEMANN THEOREM AND LOWER BOUNDS FOR GALOIS ORBITS OF SPECIAL POINTS
}

\author{
ZIYANG GAO
}

\begin{abstract}
We prove in this paper the Ax-Lindemann-Weierstraß theorem for all mixed Shimura varieties and discuss the lower bounds for Galois orbits of special points of mixed Shimura varieties. In particular we reprove a result of Silverberg [57] in a different approach. Then combining these results we prove the André-Oort conjecture unconditionally for any mixed Shimura variety whose pure part is a subvariety of $\mathcal{A}_{6}^{n}$ and under GRH for all mixed Shimura varieties of abelian type.
\end{abstract}

\section{Contents}

1. Introduction 1

2. Connected mixed Shimura varieties 6

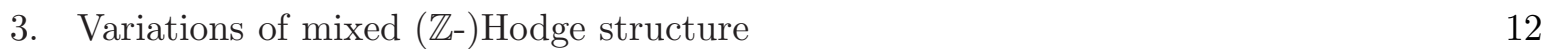

4. Realization of $\mathcal{X}^{+} \quad 14$

5. (Weakly) special subvarieties $\quad 16$

6. Algebraicity in the uniformizing space 19

7. Results for the unipotent part 20

8. The smallest weakly special subvariety containing a given subvariety 23

9. Ax-Lindemann-Weierstraß Part 1: Outline of the proof 27

10. Ax-Lindemann-Weierstraß Part 2: Estimate 33

11. Ax-Lindemann-Weierstraß Part 3: The unipotent part 36

12. Consequence of Ax-Lindemann-Weierstraß 41

13. From Ax-Lindemann-Weierstraß to André-Oort 46

14. Appendix 50

References $\quad 52$

\section{INTRODUCTION}

1.1. Background. Every connected Shimura variety, being the quotient of a Hermitian symmetric domain by an arithmetic group, can be realized as a moduli space for pure Hodge structures plus tensors. Unlike the Hermitian symmetric domains themselves, connected Shimura varieties are algebraic varieties. This was proved by Baily-Borel [7]. The prototype for all

2000 Mathematics Subject Classification. 11G18, $14 \mathrm{G} 35$. 
Shimura varieties is the Siegel moduli space of principally polarized abelian varieties of dimension $g$ with a level structure. The points in this moduli space corresponding to CM abelian varieties, which are called special points, play a particularly important role in the theory of Shimura varieties. A major reason is that the Galois actions on special points are fairly completely determined by the Shimura-Taniyama theorem [20, Theorem 4.19] and its generalization by Milne-Shih [22] to Galois conjugates of CM abelian varieties. The concept of special points and the results concerning the Galois action on them have been generalized to arbitrary Shimura varieties. Every Shimura variety has a Zariski dense subset of special points ([20, Proposition $5.2]$ ), and hence the results above have led to the concept of the canonical model of a Shimura variety over a number field: see Deligne [21] and Milne [38].

It is of course a natural problem to look for suitable compactifications of a given Shimura variety. The first compactification is the Baily-Borel (or minimal) compactification [7], which is canonical. However this compactification has bad singularities along the boundary. Next we have the toroidal compactifications [4], which are no longer canonical but provide smooth compactifications of Shimura varieties. To construct these compactifications one needs to study the boundary of a Shimura variety. As one approaches the boundary of a Hermitian symmetric domain, pure Hodge structures degenerate into mixed Hodge structures, and as one approaches the boundary of a Shimura variety, abelian varieties degenerate into 1-motives. This will lead to a new object, generalizing the notion of Shimura varieties and parametrizing variations of mixed Hodge structures (all of whose pure constituents are polarizable), which we shall call a mixed Shimura variety. In order to distinguish, we will use the term "pure Shimura variety" to denote the Shimura varieties in the first paragraph. Here we copy a list of some objects attached to a Shimura variety and the corresponding object attached to a mixed Shimura variety from Milne [38, Introduction],

\begin{tabular}{c|c} 
(pure) Shimura variety & mixed Shimura variety \\
\hline Hermitian (or bounded) symmetric domain & Siegel domain (of the third kind) \\
pure Hodge structure & mixed Hoge structure \\
reductive group over $\mathbb{Q}$ & algebraic group over $\mathbb{Q}$ with 3 -step filtration \\
abelian variety & 1-motive \\
motive & mixed motive
\end{tabular}

Here are several important examples of mixed Shimura varieties:

- the universal family of abelian varieties of dimension $g$ with a level structure;

- the $\mathbb{G}_{m}$-torsor over such a universal family which corresponds to an ample line bundle over this family;

- Poincaré bi-extension;

- products of the above.

There is also the concept of special points for mixed Shimura varieties, e.g. special points of the universal family of abelian varieties are those which correspond to torsion points on CM abelian varieties. Similar results about the Galois action on special points and the canonical models of pure Shimura varieties hold for mixed Shimura varieties: see Pink [53].

An irreducible component of a mixed Shimura subvariety of a mixed Shimura variety, or its image under a Hecke operator, is called a special subvariety. In particular, special points are precisely special subvarieties of dimension 0. As for pure Shimura varieties, every special 
subvariety contains a Zariski dense subset of special points $([53,11.7])$. The aim of this article is to study the following conjecture, which is the converse of this fact.

Conjecture 1.1 (Generalized André-Oort). Let $Y$ be a closed irreducible subvariety of a mixed Shimura variety. If $Y$ contains a Zariski-dense set of special points, then it is special.

The original André-Oort conjecture, in which one replaces "mixed Shimura variety" by "pure Shimura variety", has been established in many cases (unconditionally or under GRH without using o-minimality) by Moonen [40], André [2], Edixhoven [24, 25], Edixhoven-Yafaev [27] and Yafaev [68, 69]. It was proved under GRH by Klingler, Ullmo and Yafaev [63, 36], where equidistribution results of Clozel-Ullmo [16] were used. Later Daw [18] removed the ergodic theory from Klingler-Yafaev's result. Our generalized version of the André-Oort conjecture was suggested by Y.André in [3, Lecture 3], where he also gave a proof of the case of the universal family of elliptic curves. Results for mixed Shimura varieties have been obtained by Habegger [30] for fibered powers of elliptic surfaces. Remark that Conjecture 1.1 is not only a direct generalization of the original the André-Oort conjecture, but also contains the Manin-Mumford conjecture for any complex semi-abelian variety whose abelian variety part is CM.

1.2. Ax-Lindemann-Weierstraß. A recent development of this conjecture was made by PilaZannier. Its origin was the proof of the Manin-Mumford conjecture [52]. Afterwards using tools of o-minimality and Pila-Wilkie's counting theorem [51], Pila proved the André-Oort conjecture for $\mathcal{A}_{1}^{n}$ (product of modular curves) unconditionally [47]. Daw-Yafaev later proved the AndréOort conjecture unconditionally for Hilbert modular sufaces [19]. In this strategy of proving the André-Oort conjecture, a key step is to establish the following generalization of the functional Lindemann-Weierstrass theorem [5], which is the main result of this paper:

Theorem 1.2 (Ax-Lindemann-Weierstraß Theorem). Let $S$ be a connected mixed Shimura variety, let unif: $\mathcal{X}^{+} \rightarrow S$ be its uniformization and let $Y$ be a closed irreducible subvariety of $S$. Let $\widetilde{Z}$ be an irreducible algebraic subset of $\mathcal{X}^{+}$contained in $\operatorname{unif}^{-1}(Y)$, maximal for these properties. Then unif $(\widetilde{Z})$ is weakly special.

We explain briefly the notions in this theorem. A connected mixed Shimura variety is defined to be a connected component of a mixed Shimura variety. As for the pure case, the uniformizing space $\mathcal{X}^{+}$can be realized as an open subset (w.r.t. the archimedean topology) of a complex algebraic variety $\mathcal{X}^{\vee}(\S 4)$, and an irreducible algebraic subset of $\mathcal{X}^{+}$is defined to be an a complex analytic irreducible component of the intersection of a closed algebraic subvariety of $\mathcal{X}^{\vee}$ and $\mathcal{X}^{+}$(Definition 6.1). Consider Shimura morphisms of connected mixed Shimura varieties $T^{\prime} \stackrel{[\varphi]}{\longleftarrow} T \stackrel{[i]}{\longrightarrow} S$ and a point $t^{\prime} \in T^{\prime}$. Following Pink [54], an irreducible component of $[i]\left([\varphi]^{-1}\left(t^{\prime}\right)\right)$ is called a weakly special subvariety of $S$ (Definition 5.1). In the case of pure Shimura varieties, Moonen [40] proved that the weakly special subvarieties are precisely the totally geodesic subvarieties.

It is worth remarking that if we prove Conjecture 1.1 via Theorem 1.2 , then we also prove the Manin-Mumford conjecture for all semi-abelian varieties (not only those whose abelian variety parts are CM). Theorem 1.2 was proved for (semi-)abelian varieties by Ax [6], and then refound and reproved by Pila-Zannier [52] and Peterzil-Starchenko [46], where proofs of Manin-Mumford via Ax-Lindemann-Weierstraß can be found. For the hyperbolic case (pure Shimura varieties), this theorem has also been established in several different cases by Pila (for $\mathcal{A}_{1}^{n}[47]$ and later a 
product of universal families of elliptic curves $[48]^{1}$ ), Ullmo-Yafaev (for projective pure Shimura varieties [64]) and Pila-Tsimerman (for $\mathcal{A}_{2}$ [50] and then $\mathcal{A}_{g}$ [49]). Klingler-Ullmo-Yafaev have recently proved this theorem for all pure Shimura varieties [35] using the idea of calculating the volumes of algebraic curves near the boundary, which was firstly executed in [64] and then in [49]. Our proof is based on the result of [35]. A main ingredient to prove all the results above (including the whole Theorem 1.2) is Pila-Wilkie's counting theorem; however unlike the pure case, the "family version" of this counting theorem is crucially used in this paper. Some other difficulties to prove Theorem 1.2 for mixed Shimura varieties which we do not encounter in the pure case are listed in $\S 9$ before Lemma 9.5. We hope that this may make the strategy of our proof more clear.

We close this subsection with the following comment about weakly special subvarieties. To study them, it is useful to describe the smallest weakly special subvariety containing a given subvariety $Y$ of a connected mixed Shimura variety $S$. We shall prove in Theorem 8.1 (sometimes called $\boldsymbol{A} \boldsymbol{x}$ 's theorem of $\boldsymbol{l o g} \boldsymbol{t y p e}^{2}$ ), generalizing Moonen's result [40, 3.6, 3.7] for pure Shimura varieties, that this smallest weakly special subvariety is precisely the one defined by the connected algebraic monodromy group of $Y^{\mathrm{sm}}$. The proof uses André's [1] and Wildeshaus'[67] earlier work about variations of mixed Hodge structure (over mixed Shimura varieties). As a consequence of this description, we shall prove a characterization of weakly special subvarieties in terms of "bialgebraicity" (Corollary 8.3), which is a direct generalization of the main result of Ullmo-Yafaev $[62]$.

1.3. From Ax-Lindemann-Weierstraß to André-Oort. Ullmo and Pila-Tsimerman explained separately in [61] [49] how to deduce the André-Oort conjecture from the Ax-LindemannWeierstraß theorem for pure Shimura varieties. The proof of Ullmo is generalized to mixed Shimura varieties in this paper $(\S 12)$. They showed that in order to prove the André-Oort conjecture for pure Shimura varieties of abelian type, the only ingredient (and obstacle) left is a suitable lower bound for the Galois orbit of a special point of a pure Shimura variety conjectured by Edixhoven [26]. We prove that what we need to prove Conjecture 1.1 (for any mixed Shimura variety whose pure part is of abelian type) is the same lower bound. More explicitly, we prove that the naturally expected good lower bound for the Galois orbit of a special point, i.e. the product of the lower bounds of the base and the fiber, is fulfilled (Proposition 13.3). As a special case, this provides a new proof for the result of Silverberg [57] (Corollary 13.4)

Theorem 1.3. Let $A$ be a complex abelian variety of CM type of dimension $g$. Its field of definition $k$ is then a number field by $C M$ theory. Let $t$ be a torsion point of $A$ of order $N(t)$. If we denote by $k(t)$ the field of definition of $t$ over $k$, then $\forall \varepsilon \in(0,1)$,

$$
[k(t): k] \gg_{g, \varepsilon} N(t)^{1-\varepsilon} \text {. }
$$

In Silverberg's work, the constant on the right hand side also depends on the field $k$. The lower bound for pure Shimura varieties is known under GRH ([59], [65]). The best unconditional result is given by Tsimerman [59]. He established the lower bound unconditionally for $g \leqslant 6$ (for $g \leqslant 3$ this was also proved by Ullmo-Yafaev by a similar method [65]). Therefore as a consequence we prove (Theorem 13.6)

\footnotetext{
${ }^{1}$ The definition of weakly special subvarieties in this paper looks quite different and a lot more complicated than the one we use here. They probably coincide but I did not check it.

${ }^{2}$ This is pointed to me by Daniel Bertrand.
} 
Theorem 1.4. Under GRH, the generalized André-Oort conjecture (Conjecture (1.1)) holds for any mixed Shimura variety whose pure part is a closed subvariety of $\mathcal{A}_{g}^{n}$. This result is unconditional (i.e. we do not need GRH) if $g \leqslant 6$.

1.4. Zilber-Pink. Finally it is worth remarking that Conjecture 1.1 is part of the more general Zilber-Pink Conjecture [54, 70, 56]. Some unlikely intersections results of type André-Pink [54, Conjecture 1.6] about pure Shimura varieties beyond the André-Oort conjecture have been obtained by Pink [54, Theorem 7.6] (Galois generic points in $\mathcal{A}_{g}$ ), Habegger-Pila [31] (curves in $\mathcal{A}_{1}^{n}$ ) and Orr [43] (curves in $\mathcal{A}_{g}$ ). I shall not talk about the case of algebraic groups (see [15] for a summary). As for mixed Shimura varieties, Bertrand, Bertrand-Edixhoven, BertrandPillay and Bertrand-Masser-Pillay-Zannier have recently been working on Poincaré biextensions $[8,12,9,10,11]$. They have got several interesting results, some of which provide good examples for this paper.

Structure of this paper. In $\S 2$ we recall some basic facts about mixed Shimura varieties following Pink [53]. $\S 3$ is a summary of variations of mixed Hodge structure. In $\S 4$ we discuss the realization of the uniformizing space of any given mixed Shimura variety. In particular we give a realization of it which is at the same time semi-algebraic and complex analytic (Proposition 4.3). $\S 5$ is exploited to study (weakly) special subvarieties following Pink [54]. In $\S 6$ we define algebraic subsets of the uniformizing space and prove the functoriality of the algebraicity. In $\S 7$ we list and prove some results for the unipotent part, with the statement of the Ax-LindemannWeierstraß Theorem for the unipotent part which we will eventually prove in $\S 11$. In $\S 8$ we will have our first important results, i.e. the description of the smallest weakly special subvariety containing a given subvariety $Y$ of a connected mixed Shimura variety $S$ (Theorem 8.1) and a criterion of weakly special subvarieties in terms of "bi-algebraicity" (Corollary 8.3). The core of this paper is the proof of Theorem 1.2, and it is executed in $\S 9, \S 10$ and $\S 11$. The proof is quite technical, and for readers' convenience we organize it as follows: the outline of the proof is presented in $\S 9$, a key proposition leading to the theorem is proved in $\S 10$ by using Pila-Wilkie's counting theorem and we shall prove Ax-Lindemann-Weierstraß for the unipotent part (the fiber) separately in $\S 11$. In $\S 12$ we derive a corollary from Theorem 1.2 , which will be used to prove Theorem 1.4 in $\S 13.2$ together with a suitable lower bound discussed in $\S 13.1$. In the Appendix we reprove the Ax-Lindemann-Weierstraß theorem for algebraic tori over $\mathbb{C}$ and complex abelian varieties by this method of calculating volumes and counting points.

Acknowledgement. This topic was introduced to me by Emmanuel Ullmo. This first part of this paper $(\S 1 \sim \S 8)$ was done in Leiden University, while the two main theorems were proved when I was in Université Paris-Sud. I would like to express my gratitude to my supervisors Emmanuel Ullmo and Bas Edixhoven for weekly discussions and their valuable suggestions for the writing. I would like to thank Martin Orr for having pointed out a serious gap in $\S 9$ in a previous version as well as his several valuable remarks, especially for the last part of $\S 10$. Ya'acov Peterzil pointed out to me that the proof of the definability in $\S 10.1$ in a previous version was wrong. I have benefited a lot from the discussion with him and Sergei Starchenko for this definability problem. I also had some interesting discussion with Daniel Bertrand and Chao Zhang. Yves André, Daniel Bertrand, Bruno Klingler and Martin Orr have read a previous version of the manuscript and gave me some suggestions to improve the writing of both math 
and language. I would also like to thank them here. Finally I thank the referee for his/her careful reading and helpful suggestions thanks to which this article has been improved.

Conventions. For $x=\left(x_{1}, \ldots, x_{n}\right) \in \mathbb{Q}^{n}$, we define the height of $x$ as $H(x)=\operatorname{Max}\left(H\left(x_{1}\right), \ldots, H\left(x_{n}\right)\right)$ where for $a, b \in \mathbb{Z} \backslash\{0\}$ coprime $H(a / b)=\operatorname{Max}(|a|,|b|)$, and $H(0)=0$.

For an algebraic group $P$ over a field $k$, when we talk about a subgroup of $P$, we always mean a $k$-subgroup unless it is claimed not to be.

For the theory of o-minimality, we refer to $[64, \S 3]$ (for a concise version) and $[47, \S 2,3]$ (for a more detailed version). In this paper, "semi-algebraic" will always mean $\mathbb{R}$-semi-algebraic. The o-minimal structure we consider is $\mathbb{R}_{a n \text {,exp }}$, i.e. by saying a set $A$ is definable we mean that $A$ is definable in $\mathbb{R}_{a n, \exp }$.

\section{Connected mixed Shimura varieties}

2.1. Definition and basic properties. (cf. [53, Chapter $1,2,3])$ Let $\mathbb{S}:=\operatorname{Res}_{\mathbb{C} / \mathbb{R}}\left(\mathbb{G}_{m}\right)$ be the Deligne-torus.

Definition 2.1. A mixed Shimura datum $(P, \mathcal{X}, h)$ is a triple where

- $P$ is a connected linear algebraic group over $\mathbb{Q}$ with unipotent radical $W$ and with another algebraic subgroup $U \subset W$ which is normal in $P$ and uniquely determined by $\mathcal{X}$ using condition (3) below;

- $\mathcal{X}$ is a left homogeneous space under the subgroup $P(\mathbb{R}) U(\mathbb{C}) \subset P(\mathbb{C})$, and $\mathcal{X} \stackrel{h}{\rightarrow}$ $\operatorname{Hom}\left(\mathbb{S}_{\mathbb{C}}, P_{\mathbb{C}}\right)$ is a $P(\mathbb{R}) U(\mathbb{C})$-equivariant map s.t. every fibre of $h$ consists of at most finitely many points,

such that for some (equivalently for all) $x \in \mathcal{X}$,

(1) the composite homomorphism $\mathbb{S}_{\mathbb{C}} \stackrel{h_{x}}{\longrightarrow} P_{\mathbb{C}} \rightarrow(P / U)_{\mathbb{C}}$ is defined over $\mathbb{R}$,

(2) the adjoint representation induces on Lie $P$ a rational mixed Hodge structure of type

$$
\{(-1,1),(0,0),(1,-1)\} \cup\{(-1,0),(0,-1)\} \cup\{(-1,-1)\},
$$

(3) the weight filtration on Lie $P$ is given by

$$
W_{n}(\operatorname{Lie} P)=\left\{\begin{array}{ll}
0 & \text { if } n<-2 \\
\operatorname{Lie} U & \text { if } n=-2 \\
\operatorname{Lie} W & \text { if } n=-1 \\
\operatorname{Lie} P & \text { if } n \geqslant 0
\end{array},\right.
$$

(4) the conjugation by $h_{x}(\sqrt{-1})$ induces a Cartan involution on $G_{\mathbb{R}}^{\mathrm{ad}}$ where $G:=P / W$, and $G^{\text {ad }}$ possesses no $\mathbb{Q}$-factor $H$ s.t. $H(\mathbb{R})$ is compact,

(5) $P / P^{\text {der }}=Z(G)$ is an almost direct product of a $\mathbb{Q}$-split torus with a torus of compact type defined over $\mathbb{Q}$.

In practice, we often omit the map " $h$ " and write a mixed Shimura datum as a pair $(P, \mathcal{X})$. If in addition $P$ is reductive, then $(P, \mathcal{X})$ is called a pure Shimura datum.

Remark 2.2. (1) Let $\omega: \mathbb{G}_{m, \mathbb{R}} \hookrightarrow \mathbb{S}$ be $t \in \mathbb{R}^{*} \mapsto t \in \mathbb{C}^{*}$. Conditions (2) and (3) together imply that the composite homomorphism $\mathbb{G}_{m, \mathbb{C}} \stackrel{\omega}{\rightarrow} \mathbb{S}_{\mathbb{C}} \stackrel{h_{x}}{\rightarrow} P_{\mathbb{C}} \rightarrow(P / U)_{\mathbb{C}}$ is a co-character 
of the center of $P / W$ defined over $\mathbb{R}$. This map is called the weight. Furthermore, condition (5) implies that the weight is defined over $\mathbb{Q}$.

(2) Condition (5) also implies that every sufficiently small congruence subgroup $\Gamma$ of $P(\mathbb{Q})$ is contained in $P^{\mathrm{der}}(\mathbb{Q})$ (cf $[53$, the proof of 3.3(a)]). Fix a Levi decomposition $P=W \rtimes G$ (55, Theorem 2.3]), then $P^{\mathrm{der}}=W \rtimes G^{\mathrm{der}}$, and hence for any congruence subgroup $\Gamma<P^{\operatorname{der}}(\mathbb{Q}), \Gamma$ is Zariski dense in $P^{\text {der }}$ by condition (4) ([55, Theorem 4.10]).

(3) Condition (5) in the definition does not make the situation less general because we are only interested in a connected component of $\mathcal{X}([53,1.29])$.

Definition 2.3. Let $(P, \mathcal{X})$ be a mixed Shimura datum and let $K$ be an open compact subgroup of $P\left(\mathbb{A}_{f}\right)$ where $\mathbb{A}_{f}$ is the ring of finite adèle of $\mathbb{Q}$. The corresponding mixed Shimura variety is defined as

$$
M_{K}(P, \mathcal{X}):=P(\mathbb{Q}) \backslash \mathcal{X} \times P\left(\mathbb{A}_{f}\right) / K,
$$

where $P(\mathbb{Q})$ acts diagonally on both factors on the left and $K$ acts on $P\left(\mathbb{A}_{f}\right)$ on the right.

In this article, we only consider connected mixed Shimura data and connected mixed Shimura varieties except in $\S 13$.

Definition 2.4. $\quad$ (1) A connected mixed Shimura datum is a pair $\left(P, \mathcal{X}^{+}\right)$satisfying the conditions of Definition 2.1, where $\mathcal{X}^{+} \stackrel{h}{\longrightarrow} \operatorname{Hom}\left(\mathbb{S}_{\mathbb{C}}, P_{\mathbb{C}}\right)$ is an orbit under the subgroup $P(\mathbb{R})^{+} U(\mathbb{C}) \subset P(\mathbb{C})$.

(2) A connected mixed Shimura variety $S$ associated with $\left(P, \mathcal{X}^{+}\right)$is of the form $\Gamma \backslash \mathcal{X}^{+}$ for some congruence subgroup $\Gamma \subset P(\mathbb{Q}) \cap P(\mathbb{R})_{+}$, where $P(\mathbb{R})_{+}$is the stabilizer of $\mathcal{X}^{+} \subset \operatorname{Hom}_{\mathbb{C}}\left(\mathbb{S}_{\mathbb{C}}, P_{\mathbb{C}}\right)$.

Every connected mixed Shimura variety is a connected component of a mixed Shimura variety, and vice versa $([53,3.2])$. A connected mixed Shimura variety is a complex analytic space with at most finite quotient singularities, and if $\Gamma$ is sufficiently small (for example if $\Gamma$ is neat), then $\Gamma \backslash \mathcal{X}^{+}$is smooth. For details we refer to [54, Fact 2.3] or [53, 1.18, 3.3, 9.24].

Recall the following definition, which Pink calls "irreducible" in [53, 2.13].

Definition 2.5. A connected mixed Shimura datum $\left(P, \mathcal{X}^{+}\right)$is said to have generic MumfordTate group if $P$ possesses no proper normal subgroup $P^{\prime}$ defined over $\mathbb{Q}$ s.t. for one (equivalently all) $x \in \mathcal{X}^{+}, h_{x}$ factors through $P_{\mathbb{C}}^{\prime} \subset P_{\mathbb{C}}$. We shall denote this case by $P=\operatorname{MT}\left(\mathcal{X}^{+}\right)$. (This terminology will be explained in Remark 3.8).

Proposition 2.6. Let $\left(P, \mathcal{X}^{+}\right)$be a connected mixed Shimura datum, then

(1) there exists a connected mixed Shimura datum $\left(P^{\prime}, \mathcal{X}^{\prime+}\right) \hookrightarrow\left(P, \mathcal{X}^{+}\right)$s.t. $P^{\prime}=\operatorname{MT}\left(\mathcal{X}^{\prime+}\right)$ and $\mathcal{X}^{\prime+} \simeq \mathcal{X}^{+}$under this embedding;

(2) if $\left(P, \mathcal{X}^{+}\right)$has generic Mumford-Tate group, then $P$ acts on $U$ via a scalar. In particular, any subgroup of $U$ is normal in $P$.

Proof. [53, 2.13, 2.14].

Definition 2.7. A (Shimura) morphism of connected mixed Shimura data $\left(Q, \mathcal{Y}^{+}\right) \rightarrow\left(P, \mathcal{X}^{+}\right)$ is a homomorphism $\varphi: Q \rightarrow P$ of algebraic groups over $\mathbb{Q}$ which induces a map $\mathcal{Y}^{+} \rightarrow \mathcal{X}^{+}$, $y \mapsto \varphi \circ y$. A Shimura morphism of connected mixed Shimura varieties is a morphism of varieties induced by a Shimura morphism of connected mixed Shimura data. 
Fact 1. (cf $[53,2.9])$ Let $\left(P, \mathcal{X}^{+}\right)$be a connected mixed Shimura datum and let $P_{0}$ be a normal subgroup of $P$. Then there exists a quotient connected mixed Shimura datum $\left(P, \mathcal{X}^{+}\right) / P_{0}$ and a morphism $\left(P, \mathcal{X}^{+}\right) \rightarrow\left(P, \mathcal{X}^{+}\right) / P_{0}$ unique up to isomorphism s.t. every morphism $\left(P, \mathcal{X}^{+}\right) \rightarrow$ $\left(P^{\prime}, \mathcal{X}^{+\prime}\right)$, where the homomorphism $P \rightarrow P^{\prime}$ factors through $P / P_{0}$, factors in a unique way through $\left(P, \mathcal{X}^{+}\right) / P_{0}$. Such a Shimura morphism $\left(P, \mathcal{X}^{+}\right) \rightarrow\left(P, \mathcal{X}^{+}\right) / P_{0}$ is called a quotient Shimura morphism.

Notation 2.8. For convenience, we fix some notation here. Given a connected mixed Shimura datum $\left(P, \mathcal{X}^{+}\right)$, we always denote by $W=\mathcal{R}_{u}(P)$ the unipotent radical of $P, G:=P / W$ the reductive part, $U \triangleleft P$ the weight -2 part, $V:=W / U$ the weight -1 part and $\left(P / U, \mathcal{X}_{P / U}^{+}\right):=$ $\left(P, \mathcal{X}^{+}\right) / U$ (resp. $\left.\left(G, \mathcal{X}_{G}^{+}\right):=\left(P, \mathcal{X}^{+}\right) / W\right)$ the corresponding connected mixed Shimura datum whose weight -2 part is trivial (resp. pure Shimura datum). If we have several connected mixed Shimura data, say $\left(P, \mathcal{X}^{+}\right)$and $\left(Q, \mathcal{Y}^{+}\right)$, then we distinguish the different parts associated with them by adding subscript $W_{P}, W_{Q}, G_{P}, G_{Q}$, etc. For a connected mixed Shimura variety $S$, we denote by $S_{P / U}$ (resp. $\left.S_{G}\right)$ its image under the Shimura morphism induced by $\left(P, \mathcal{X}^{+}\right) \rightarrow$ $\left(P / U, \mathcal{X}_{P / U}^{+}\right)\left(\right.$resp. $\left.\left(P, \mathcal{X}^{+}\right) \rightarrow\left(G, \mathcal{X}_{G}^{+}\right)\right)$.

Proposition 2.9. Let $(Q, \mathcal{Y}) \stackrel{f}{\rightarrow}(P, \mathcal{X})$ be a Shimura morhpism, then $f\left(W_{Q}\right) \subset W_{P}$ (resp. $\left.f\left(U_{Q}\right) \subset f\left(U_{P}\right)\right)$, and hence $f$ induces

$$
\bar{f}:\left(G_{Q}, \mathcal{Y}_{G_{Q}}\right) \rightarrow\left(G_{P}, \mathcal{X}_{G_{P}}\right) \quad\left(\text { resp. } \bar{f}^{\prime}:\left(Q / Q_{U}, \mathcal{Y}_{Q / U_{Q}}\right) \rightarrow\left(P / U_{P}, \mathcal{X}_{P / U_{P}}\right)\right) .
$$

Furthermore, if the underlying homomorphism of algebraic groups $f$ is injective, then so are $\bar{f}$ and $\bar{f}^{\prime}$.

Proof. Since

$$
\text { Lie } W_{P}=W_{-1}(\operatorname{Lie} P) \quad \text { and } \quad \operatorname{Lie} W_{Q}=W_{-1}(\operatorname{Lie} Q),
$$

by the following commutative diagram

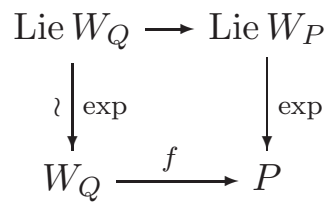

(here exp is algebraic and is an isomorphism as a morphism between algebraic varieties because $W_{Q}$ is unipotent), $f\left(W_{Q}\right) \subset W_{P}$.

Hence $f$ induces a map $G_{Q} \rightarrow G_{P}$. Now the existence of $\bar{f}$ follows from the universal property of the quotient Shimura datum $([53,2.9])$.

Furthermore, suppose now that $f$ is injective. By Levi decomposition, the exact sequence

$$
1 \rightarrow W_{Q} \rightarrow Q \stackrel{\pi_{Q}}{\longrightarrow} G_{Q} \rightarrow 1
$$

splits. Choose a splitting $s_{Q}: G_{Q} \rightarrow Q$, then we have the following diagram whose solid arrows commute:

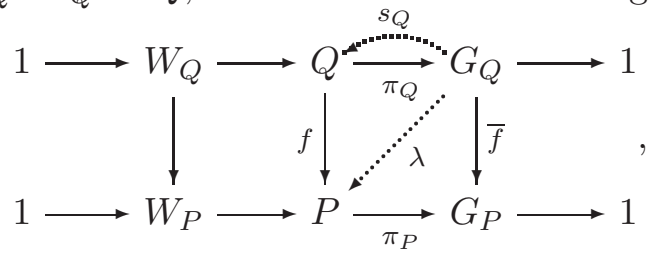


where $\lambda:=f \circ s_{Q}$. Then $\lambda$ is injective since $f, s_{Q}$ are. And $\pi_{P} \circ \lambda=\pi_{P} \circ f \circ s_{Q}=\bar{f} \circ \pi_{Q} \circ s_{Q}=\bar{f}$, so we have

$$
\operatorname{Ker}(\bar{f})=G_{Q} \cap W_{P}
$$

where the intersection is taken in $P$. $\left(G_{Q} \cap W_{P}\right)^{\circ}$ is smooth (since we are in the characteristic 0 ), connected unipotent (since it is in $W_{P}$ ) and normal in $G_{Q}$ (since $W_{P}$ is normal in $P$ ), so it is trivial since $G_{Q}$ is reductive. So $G_{Q} \cap W_{P}$ is finite, hence trivial because $W_{P}$ is unipotent over $\mathbb{Q}$. To sum it up, $\bar{f}$ is injective.

The proof for the statements with $U$ 's is similar.

2.2. Structure of the underlying group. (cf $[53,2.15])$.

For a given connected mixed Shimura datum $\left(P, \mathcal{X}^{+}\right)$, we can associate to $P$ a 4 -tuple $(G, V, U, \Psi)$ which is defined as follows:

- $G:=P / \mathcal{R}_{u}(P)$ is the reductive part of $P$;

- $U$ is the normal subgroup of $P$ as in Definition 2.1 and $V:=\mathcal{R}_{u}(P) / U$. Both of them are vector groups with an action of $G$ induced by conjugation in $P$ (which factors through $G$ for reason of weight);

- The commutator on $W:=\mathcal{R}_{u}(P)$ induces a $G$-equivariant alternating form $\Psi: V \times V \rightarrow$ $U$ by reason of weight as explained by Pink in [53, 2.15]. Moreover, $\Psi$ is given by a polynomial with coefficients in $\mathbb{Q}$.

On the other hand, $P$ is uniquely determined up to isomorphism by this 4-tuple in the following sense:

- let $W$ be the central extension of $V$ by $U$ defined by $\Psi$. More concretely, $W=U \times V$ as a $\mathbb{Q}$-variety and the group law on $W$ is (this can be proved using the Baker-CampbellHausdorff formula)

$$
(u, v)\left(u^{\prime}, v^{\prime}\right)=\left(u+u^{\prime}+\frac{1}{2} \Psi\left(v, v^{\prime}\right), v+v^{\prime}\right)
$$

- define the action of $G$ on $W$ by $g((u, v)):=(g u, g v)$;

- define $P:=W \rtimes G$.

2.3. Siegel type. (cf $[53,2.7,2.25]$ for mixed Shimura data of Siegel type and [53, 10.1-10.14] for mixed Shimura varieties of Siegel type)

Let $g \in \mathbb{N}_{>0}$. Let $V_{2 g}$ be a $\mathbb{Q}$-vector space of dimension $2 g$ and let

$$
\Psi: V_{2 g} \times V_{2 g} \rightarrow U_{2 g}:=\mathbb{G}_{a, \mathbb{Q}}
$$

be a non-degenerate alternating form. Define

$$
\mathrm{GSp}_{2 g}:=\left\{g \in \mathrm{GL}\left(V_{2 g}\right) \mid \Psi\left(g v, g v^{\prime}\right)=\nu(g) \Psi\left(v, v^{\prime}\right) \text { for some } \nu(g) \in \mathbb{G}_{m}\right\},
$$

and $\mathbb{H}_{g}$ the set of all homomorphisms

$$
\mathbb{S} \rightarrow \mathrm{GSp}_{2 g, \mathbb{R}}
$$

which induce a pure Hodge structure of type $\{(-1,0),(0,-1)\}$ on $V_{2 g}$ and for which either $\Psi$ or $-\Psi$ defines a polarization. Let $\mathbb{H}_{g}^{+}$be the set of all such homomorphisms s.t. $\Psi$ defines a polarization. 
$\mathrm{GSp}_{2 g}$ acts on $U_{2 g}$ by the scalar $\nu$, which induces a pure Hodge structure of type $(-1,-1)$ on $U_{2 g}$. Let $W_{2 g}$ be the central extension of $V_{2 g}$ by $U_{2 g}$ defined by $\Psi$, then the action of $\mathrm{GSp}_{2 g}$ on $W_{2 g}$ induces a Hodge structure of type $\{(-1,0),(0,-1),(-1,-1)\}$ on $W_{2 g}$.

By $[53,2.16,2.17]$, there exist connected mixed Shimura data $\left(P_{2 g, a}, \mathcal{X}_{2 g, a}^{+}\right)$and $\left(P_{2 g}, \mathcal{X}_{2 g}^{+}\right)$, where $P_{2 g, a}:=V_{2 g} \rtimes \mathrm{GSp}_{2 g}$ and $P_{2 g}:=W_{2 g} \rtimes \mathrm{GSp}_{2 g}$.

Definition 2.10. The connected mixed Shimura data $\left(\mathrm{GSp}_{2 g}, \mathbb{H}_{g}^{+}\right),\left(P_{2 g, a}, \mathcal{X}_{2 g, a}^{+}\right)$and $\left(P_{2 g}, \mathcal{X}_{2 g}^{+}\right)$ are called of Siegel type (of genus $g$ ).

Next we introduce connected mixed Shimura varieties of Siegel type. They have good modular interpretation ([53, 10.8-10.14]).

For $N \geqslant 4$ and even, define

$$
\Gamma_{\mathrm{GSp}}(N):=\left\{g \in \mathrm{GSp}_{2 g}(\mathbb{Z}) \mid g \equiv 1 \bmod N\right\}
$$

and

$$
\Gamma_{W}(N):=\left(N \cdot U_{2 g}(\mathbb{Z})\right) \times\left(N \cdot V_{2 g}(\mathbb{Z})\right)
$$

under the identification $W \simeq U \times V$ in $\S 2.2 . \Gamma_{W}(N)$ is indeed a subgroup of $W(\mathbb{Z})$ by the group operation (defined by $\Psi$ ). Let $\Gamma_{V}(N):=N \cdot V_{2 g}(\mathbb{Z})$, and write

$$
\begin{array}{r}
\mathcal{A}_{g}(N):=\Gamma_{\mathrm{GSp}}(N) \backslash \mathbb{H}_{g}^{+} \\
\mathfrak{A}_{g}(N):=\left(\Gamma_{V}(N) \rtimes \Gamma_{\mathrm{GSp}}(N)\right) \backslash \mathcal{X}_{2 g}^{+}, \\
\mathfrak{L}_{g}(N):=\left(\Gamma_{W}(N) \rtimes \Gamma_{\mathrm{GSp}}(N)\right) \backslash \mathcal{X}_{2 g}^{+},
\end{array}
$$

Then $\mathcal{A}_{g}(N)$ is a moduli space of abelian varieties of dimension $g$ with a level structure, $\mathfrak{A}_{g}(N) \rightarrow$ $\mathcal{A}_{g}(N)$ is the universal family of abelian varieties (and hence a principally polarized abelian scheme of relative dimension $g$ ), and $\mathfrak{L}_{g}(N) \rightarrow \mathfrak{A}_{g}(N)$ is a $\mathbb{G}_{m}$-torsor which (up to replacing the $\mathbb{G}_{m}$-action by its inverse) corresponds to a relatively ample line bundle over $\mathfrak{A}_{g}(N) \rightarrow \mathcal{A}_{g}(N)$. For more details see $[53,10.5,10.9,10.10]$.

Definition 2.11. The connected mixed Shimura varieties $\mathcal{A}_{g}(N), \mathfrak{A}_{g}(N)$ and $\mathfrak{L}_{g}(N)$ are called of Siegel type of level $N$ (and of genus $g$ ).

Denote by $\mathrm{GSp}_{0}:=\mathbb{G}_{m}$ and $P_{0}:=\mathbb{G}_{a} \rtimes \mathbb{G}_{m}$ with the standard action of $\mathbb{G}_{m}$ on $\mathbb{G}_{a}$. Pink proved the following lemma $([53,2.26])$

Lemma 2.12 (Reduction Lemma). Let $\left(P, \mathcal{X}^{+}\right)$be a connected mixed Shimura datum with generic Mumford-Tate group.

(1) If $V$ is trivial, then there exists an embedding

$$
\left(P, \mathcal{X}^{+}\right) \hookrightarrow\left(G_{0}, \mathcal{D}^{+}\right) \times \prod_{i=1}^{r}\left(P_{0}, \mathcal{X}_{0}^{+}\right)
$$

where $r=\operatorname{dim}(U)$ (see $[53,2.8,2.14]$ for definition of $\left.\left(P_{0}, \mathcal{X}_{0}^{+}\right)\right)$;

(2) If $V$ is not trivial, then there exist a connected pure Shimura datum $\left(G_{0}, \mathcal{D}^{+}\right)$and Shimura morphisms

$$
\begin{aligned}
& \left(P^{\prime}, \mathcal{X}^{\prime+}\right) \rightarrow\left(P, \mathcal{X}^{+}\right) \\
& \text {and }\left(P^{\prime}, \mathcal{X}^{\prime+}\right) \stackrel{\lambda}{\rightarrow}\left(G_{0}, \mathcal{D}^{+}\right) \times \prod_{i=1}^{r}\left(P_{2 g}, \mathcal{X}_{2 g}^{+}\right)
\end{aligned}
$$


s.t. $\operatorname{Ker}\left(P^{\prime} \rightarrow P\right)$ is of dimension 1 and of weight-2. Moreover $\left.\lambda\right|_{V}: V \simeq V_{2 g} \rightarrow \oplus_{i=1}^{r} V_{2 g}$ is the diagonal map, $\left.\lambda\right|_{U^{\prime}}: U^{\prime} \simeq \oplus_{i=1}^{r} U_{2 g}$ and $G \stackrel{\left.\lambda\right|_{G}}{\longrightarrow} G_{0} \times \prod_{i=1}^{r} \mathrm{GSp}_{2 g} \rightarrow \mathrm{GSp}_{2 g}$ is nontrivial for each projection.

Proof. The statement except the last claim of the "Moreover" part is $[53,2.26$ statement \& pp 45]. For the last part, call $p_{i}: G \rightarrow \mathrm{GSp}_{2 g}$ the composite with the i-th projection. If $p_{i}$ is trivial, then $p_{i}\left(P^{\prime}, \mathcal{X}^{\prime+}\right)$ is trivial since a connected mixed Shimura datum is trivial if its pure part is trivial. This contradicts the dimension of $V$.

2.4. Decomposition of $V$ and $U$ as $G$-modules. We start this subsection by the following group theoretical proposition.

Proposition 2.13. Let $1 \rightarrow N \rightarrow Q \stackrel{\varphi}{\rightarrow} Q^{\prime} \rightarrow 1$ be an exact sequence of algebraic groups over $\mathbb{Q}$. Then the following diagram with solid arrows is commutative and all the lines and columns are exact:

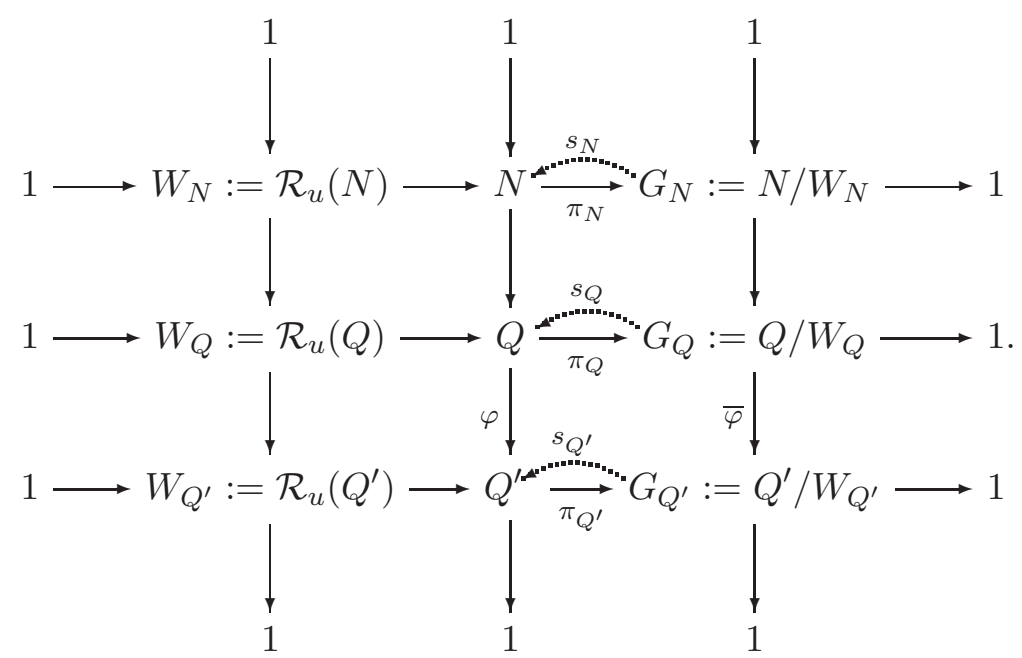

Moreover, if we fix a morphism $s_{Q}$ which splits the middle line (such an $s_{Q}$ exists by Levi decomposition), then we can deduce $s_{N}$ and $s_{Q^{\prime}}$ which split the other two lines. Note that in this case, the action of $G_{N}$ on $W_{Q^{\prime}}$ induced by $s_{Q}$ is trivial.

Proof. The two bottom lines are already exact. By group theory, we know $\varphi\left(W_{Q}(\overline{\mathbb{Q}})\right)=W_{Q^{\prime}}(\overline{\mathbb{Q}})$ ([14, Corollary 14.11]), and since the set of closed points of $W_{Q}$ (resp. $W_{Q^{\prime}}$ ) is dense on $W_{Q}$ (resp. $\left.W_{Q^{\prime}}\right)$, we have $\varphi\left(W_{Q}\right)=W_{Q^{\prime}}$. In consequence, we have the map $\bar{\varphi}$, which is surjective since $\varphi$ is. Now we get the solid diagram by snake-lemma. $G_{N}$ is reductive $([14,14.2$ Corollary $(\mathrm{b})])$.

If we have an $s_{Q}$, then to get a desired $s_{Q^{\prime}}$ (and $\left.s_{N}\right)$ is equivalent to prove that $\varphi \circ s_{Q}\left(G_{N}\right)$ is trivial, i.e. the intersection of this image with $W_{Q^{\prime}}$ (in $Q^{\prime}$ ) is trivial and the projection of this image to $G_{Q^{\prime}}$ (under $\pi_{Q^{\prime}}$ ) is trivial. The projection is trivial by a simple diagram-chasing. The neutral component of the intersection is trivial since it is reductive and unipotent, and hence the intersection is trivial since $W_{Q^{\prime}}$ is unipotent over $\mathbb{Q}$. Now the triviality of the action of $G_{N}$ on $W_{Q^{\prime}}$ induced by $s_{Q}$ is automatic.

Corollary 2.14. Let $\left(P, \mathcal{X}^{+}\right)$be a connected mixed Shimura datum. Suppose $N \triangleleft P$. Then there are decompositions

$$
V=V_{N} \oplus V_{N}^{\perp} \quad\left(\text { resp. } U=U_{N} \oplus U_{N}^{\perp}\right)
$$


as $G$-modules, where $V_{N}:=V \cap N$ (resp. $\left.U_{N}:=U \cap N\right)$, s.t. the action of $G_{N}:=N / V_{N}$ on $V_{N}^{\perp}$ (resp. $U_{N}^{\perp}$ ) is trivial.

Proof. To prove the decomposition of $V$, apply Proposition 2.13 to the exact sequence

$$
1 \rightarrow V_{N} \rtimes G_{N} \rightarrow V \rtimes G \rightarrow\left(V / V_{N}\right) \rtimes\left(G / G_{N}\right) \rightarrow 1,
$$

then since $G$ is reductive, the vertical line on the left (in the diagram of the proposition) splits. The conjugation by $P$ on $V$ factors through $G$ by reason of weights, and hence equals to the action of $G$ on $V$ induced by any Levi decomposition $s_{P}$. So the action of $G_{N}$ on $V_{N}^{\perp}$ is trivial by the last assertion of Proposition 2.13.

To prove the decomposition of $U$, it suffices to apply Proposition 2.13 to the exact sequence

$$
1 \rightarrow U_{N} \rtimes G_{N} \rightarrow U \rtimes G \rightarrow\left(U / U_{N}\right) \rtimes\left(G / G_{N}\right) \rightarrow 1 .
$$

In fact we have a better result if $\left(P, \mathcal{X}^{+}\right)$has generic Mumford-Tate group.

Proposition 2.15. Let $\left(P, \mathcal{X}^{+}\right)$be a connected mixed Shimura datum s.t. $P=\mathrm{MT}\left(\mathcal{X}^{+}\right)$. Suppose $N \triangleleft P$ s.t. $N$ possesses no non-trivial torus quotient. Then $G_{N}$ acts trivially on $U$.

Proof. By Reduction Lemma (Lemma 2.12), we may assume that $\left(P, \mathcal{X}^{+}\right) \hookrightarrow\left(G_{0}, \mathcal{D}^{+}\right) \times$ $\prod_{i=1}^{r}\left(P_{2 g}, \mathcal{X}_{2 g}^{+}\right)(g \geqslant 0)$. Since $N$ possesses no non-trivial torus quotient, $G_{N}$ is semi-simple (the last line of the proof of Proposition 5.4). So

$$
G_{N}=G_{N}^{\text {der }}<G^{\text {der }}<\left(G_{0} \times \prod_{i=1}^{r} \mathrm{GSp}_{2 g}\right)^{\text {der }}=G_{0}^{\text {der }} \times \prod_{i=1}^{r} \mathrm{Sp}_{2 g}
$$

where $\operatorname{Sp}_{0}:=1$. Hence $G_{N}$ acts trivially on $U$ since $G_{0}^{\text {der }} \times \prod_{i=1}^{r} \operatorname{Sp}_{2 g}$ acts trivially on $\oplus_{i=1}^{r} U_{2 g}$.

\section{Variations of MiXed (ZZ-)Hodge structure}

\subsection{Arbitrary variation of mixed Hodge structure.}

Definition 3.1. ([44, Definition 14.44]) Let $S$ be a complex manifold. A variation of mixed Hodge structure on $S$ is a triple $(\mathbb{V}, W ., \mathcal{F})$ with

(1) a local system $\mathbb{V}$ of free $\mathbb{Z}$-module of finite rank on $S$;

(2) a finite increasing filtration $\left\{W_{m}\right\}$ of the local system $\mathbb{V}_{\mathbb{Q}}:=\mathbb{V} \otimes_{\mathbb{Z}} \mathbb{Q}$ by local sub-systems (this is called the weight filtration);

(3) a finite decreasing filtration $\left\{\mathcal{F}^{p}\right\}$ of the holomorphic vector bundle $\mathcal{V}:=\mathbb{V} \otimes_{\mathbb{Z}} \mathcal{O}_{S}$ by holomorphic sub-bundles (this is called the Hodge filtration).

s.t.

(1) for each $s \in S$, the filtrations $\left\{\mathcal{F}^{p}(s)\right\}$ and $\left\{W_{m}\right\}$ of $\mathbb{V}(s) \simeq \mathbb{V}_{s} \otimes_{\mathbb{Z}} \mathbb{C}$ define a mixed Hodge structure on the $\mathbb{Z}$-module of finite rank $\mathbb{V}_{s}$;

(2) the connection $\nabla: \mathcal{V} \rightarrow \mathcal{V} \otimes_{\mathcal{O}_{S}} \Omega_{S}^{1}\left(\mathcal{V}:=\mathbb{V} \otimes_{\mathbb{Z}} \mathcal{O}_{S}\right)$ whose sheaf of horizontal sections is $\mathbb{V}_{\mathbb{C}}$ satisfies the Griffiths' transversality condition

$$
\nabla\left(\mathcal{F}^{p}\right) \subset \mathcal{F}^{p-1} \otimes \Omega_{S}^{1} .
$$


Definition 3.2. A variation of mixed Hodge structure on $S$ is called (graded-)polarizable if the induced variations of pure Hodge structure $\mathrm{Gr}_{k}^{W} \mathbb{V}$ are all polarizable, i.e. for each $k$, there exists a flat morphism of variations

$$
Q_{k}: \mathrm{Gr}_{k}^{W} \mathbb{V} \otimes \mathrm{Gr}_{k}^{W} \mathbb{V} \rightarrow \mathbb{Z}(-k)_{S}
$$

which induces on each fibre a polarization of the corresponding Hodge structure of weight $k$.

Let $\pi: \widetilde{S} \rightarrow S$ be a universal covering and choose a trivialization $\pi^{*} \mathbb{V} \simeq \widetilde{S} \times V$. For $s \in S$, $\mathrm{MT}_{s} \subset \mathrm{GL}\left(\mathcal{V}_{s}\right)$ denote the Mumford-Tate group of its fibre. The choice of a point $\widetilde{s} \in \widetilde{S}$ with $\pi(\widetilde{s})=s$ gives an identification $\mathcal{V}_{s} \simeq V$, whence an injective homomorphism $i_{\widetilde{s}}: \operatorname{MT}_{s} \hookrightarrow \operatorname{GL}(V)$. By $\left[1, \S 4\right.$, Lemma 4], on $S^{\circ}:=S \backslash \Sigma$ where $\Sigma$ is a meager subset of $S, M:=\operatorname{Im}\left(i_{\widetilde{s}}\right) \subset \operatorname{GL}(V)$ does not depend on $s$, nor on the choice of $\widetilde{s}$. We call $S^{\circ}$ the "Hodge-generic" locus and the group $M$ the generic Mumford-Tate group of $\left(\mathbb{V}, W ., \mathcal{F}^{*}\right)$.

On the other hand, if we choose a base-point $s \in S$ and a point $\widetilde{s} \in \widetilde{S}$ with $\pi(\widetilde{s})=s$, then then local system $\mathbb{V}$ corresponds to a representation $\rho: \pi_{1}(S, s) \rightarrow \mathrm{GL}(V)$, called the monodromy representation. The algebraic monodromy group is defined as the smallest algebraic subgroup of $\mathrm{GL}(V)$ over $\mathbb{Q}$ which contains the image of $\rho$. We write $H_{s}^{\text {mon }}$ for its connected component of the identity, called the connected algebraic monodromy group. Given the trivialization of $\pi^{*} \mathbb{V}$, the group $H_{s}^{\text {mon }} \subset \mathrm{GL}(V)$ is independent of the choice of $s$ and $\widetilde{s}$.

Suppose now that $\left(\mathbb{V}, W, \mathcal{F}^{\cdot}\right)$ is (graded-)polarizable, then $H_{s}^{\text {mon }}<M$ for any $s \in S^{\circ}$ by $[1$, $\S 4$, Lemma 4].

3.2. Admissible variations of mixed Hodge structure. We now recall the concept of "admissible" variations of mixed Hodge structure which was introduced by Steenbrick-Zucker [58] and studied by Kashiwara [34] and Hain-Zucker [32]. We give the definition here, but instead of the exact definition, we shall only use the notion of "admissibility" and the fact that it can be defined using "curve test". We will use $\Delta$ (resp. $\Delta^{*}$ ) to denote the unit disc (resp. punctured unit disc).

Definition 3.3. (see [44, Definition 14.49])

(1) A variation of mixed Hodge structure $\left(\mathbb{V}, W, \mathcal{F}^{*}\right)$ over the punctured unit disc $\Delta^{*}$ is called admissible if

- it is (graded-)polarizable;

- the monodromy $T$ is unipotent and the weight filtration $M(N, W$.) of $N:=\log T$ relative to $W$. exists;

- the filtration $\mathcal{F}$ extends to a filtration $\widetilde{\mathcal{F}}$ of $\widetilde{\mathcal{V}}$ which induced ${ }^{k} \widetilde{\mathcal{F}}$ on $\operatorname{Gr}_{k}^{W} \widetilde{\mathcal{V}}$ for each $k$.

(2) Let $S$ be a smooth connected complex algebraic variety and let $\bar{S}$ be a compactification of $S$ s.t. $\bar{S} \backslash S$ is a normal crossing divisor. A (graded-)polarizable variation of mixed Hodge structure $(\mathbb{V}, W ., \mathcal{F})$ on $S$ is called admissible if for every holomorphic map $i: \Delta \rightarrow \bar{S}$ which maps $\Delta^{*}$ to $S$ and s.t. $i^{*} \mathbb{V}$ has unipotent monodromy, the variation $i^{*}\left(\mathbb{V}, W ., \mathcal{F}^{*}\right)$ is admissible. (This definition is sometimes called the "curve test" version).

Remark 3.4. This definition is equivalent to the one given in $[32,1.5]$. See [58, Properties 3.13 \& Appendix], [34, 11 \& Theorem 4.5.2] and [32, 1.5] for details.

The following lemma is an easy property of admissibility and is surely known by many people, but I cannot find any reference, so I give a proof here. 
Lemma 3.5. Let $S$ be a smooth connected complex algebraic variety and let $\left(\mathbb{V}, W ., \mathcal{F}^{*}\right)$ be an admissible variation of mixed Hodge structure on $S$. Then for any smooth connected (not necessarily closed) subvariety $j: Y \hookrightarrow S, j^{*}\left(\mathbb{V}, W ., \mathcal{F}^{*}\right)$ is also admissible on $Y$.

Proof. Take smooth compactifications $\bar{Y}$ of $Y$ and $\bar{S}$ of $S$ s.t. $\bar{Y} \backslash Y$ and $\bar{S} \backslash S$ are normal crossing divisors and s.t. $j: Y \hookrightarrow S$ extends to a morphism $\bar{j}: \bar{Y} \rightarrow \bar{S}$. This can be done by first choosing any compactifications of $Y^{\mathrm{cp}}$ of $Y$ and $S^{\mathrm{cp}}$ of $S$ with normal crossing divisors and then taking a suitable resolution of singularities of the closure of the graph of $j$ in $Y^{\mathrm{cp}} \times S^{\mathrm{cp}}$. Now the conclusion follows from our "curve test" version of the definition.

\subsection{Consequences of admissibility. Y.André proved that:}

Theorem 3.6. Let $\left(\mathbb{V}, W ., \mathcal{F}^{*}\right)$ be an admissible variation of mixed Hodge structure over a smooth connected complex algebraic variety $S$, then for any $s \in S$, the connected monodromy group $H_{s}^{\text {mon }}$ is a normal subgroup of the generic Mumford-Tate group $M$ and also its derived group $M^{\mathrm{der}}$.

Proof. $\left[1, \S 5\right.$, Theorem 1] states that $H_{s}^{\text {mon }} \triangleleft M^{\text {der }}$, and in the proof he first proved that $H_{s}^{\text {mon }} \triangleleft$ $M$.

Now we state a theorem which roughly says that all the variations of mixed Hodge structure obtained from representations of the underlying group of a connected mixed Shimura datum are admissible. Explicitly, let $S$ be a connected mixed Shimura variety associated with the connected mixed Shimura datum $\left(P, \mathcal{X}^{+}\right)$and let unif : $\mathcal{X}^{+} \rightarrow S=\Gamma \backslash \mathcal{X}^{+}$be the uniformization. Suppose that $\Gamma$ is neat. Consider any $\mathbb{Q}$-representation $\xi: P \rightarrow \mathrm{GL}(V)$. By [55, Proposition 4.2], there exists a $\Gamma$-invariant lattice $V_{\mathbb{Z}}$ of $V . \xi$ and $V_{\mathbb{Z}}$ together give rise to a VMHS on $S$ whose underlying local system is $\Gamma \backslash\left(\mathcal{X}^{+} \times V_{\mathbb{Z}}\right)$. This variation is (graded-)polarizable by [53, 1.18(d)].

Theorem 3.7. Suppose that $S,\left(P, \mathcal{X}^{+}\right), \xi: P \rightarrow \mathrm{GL}(V)$ and $V_{\mathbb{Z}}$ are as in the previous paragraph, then the variation of mixed Hodge structure obtained as above is admissible.

Proof. [67, Theorem 2.2] says that the corresponding $\mathbb{Q}$-variation is admissible, and $\Gamma$ gives a $\mathbb{Z}$-structure as in the discussion above.

Remark 3.8. In this language, we can rephrase Definition 2.5 as: $P$ is the generic MumfordTate group (of the variation in Theorem 3.7). It is clear that for any Hodge generic point $x \in \mathcal{X}^{+}$, the only $\mathbb{Q}$-subgroup $N$ of $P^{\text {der }}$ s.t. $N(\mathbb{R})^{+} U_{N}(\mathbb{C})$, where $U_{N}:=U \cap N$, stabilizes $x$ is the trivial group.

\section{Realization of $\mathcal{X}^{+}$}

Let $\left(P, \mathcal{X}^{+}\right)$be a connected mixed Shimura datum. We first define the dual $\mathcal{X}^{\vee}$ of $\mathcal{X}^{+}$(see $[53,1.7(\mathrm{a})]$ or $[38$, Chapter VI, Proposition 1.3]):

Let $M$ be a faithful representation of $P$ and take any $x_{0} \in \mathcal{X}^{+}$. The weight filtration on $M$ is constant, so the Hodge filtration $x \mapsto \operatorname{Fil}_{x}\left(M_{\mathbb{C}}\right)$ gives an injective map $\mathcal{X}^{+} \hookrightarrow \operatorname{Grass}(M)(\mathbb{C})$ to a certain flag variety. In fact, this injective map factors through

$$
\mathcal{X}^{+}=P(\mathbb{R})^{+} U(\mathbb{C}) / C\left(x_{0}\right) \hookrightarrow P(\mathbb{C}) / F_{x_{0}}^{0} P(\mathbb{C}) \hookrightarrow \operatorname{Grass}(M)(\mathbb{C})
$$

where $C\left(x_{0}\right)$ is the stabilizer of $x_{0}$ in $P(\mathbb{R})^{+} U(\mathbb{C})$. The first injection is an open immersion ([53, $1.7(\mathrm{a})]$ or $\left[38\right.$, Chapter VI, (1.2.1)]). We define the dual $\mathcal{X}^{\vee}$ of $\mathcal{X}^{+}$to be

$$
\mathcal{X}^{\vee}:=P(\mathbb{C}) / F_{x_{0}}^{0} P(\mathbb{C}) .
$$


Then $\mathcal{X}^{\vee}$ is clearly a connected smooth complex algebraic variety.

Proposition 4.1. Under the open immersion $\mathcal{X}^{+} \hookrightarrow \mathcal{X}^{\vee}, \mathcal{X}^{+}$is realized as a semi-algebraic set which is also a complex manifold.

Proof. $\mathcal{X}^{+}$is smooth since it is a homogeneous space, and the open immersion endows it with a complex structure. For semi-algebraicity, consider the diagram

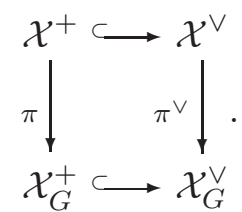

Now $\mathcal{X}^{+}=\left\{x \in \mathcal{X}^{\vee} \mid \pi^{\vee}(x) \in \mathcal{X}_{G}^{+}\right\}$and $\pi^{\vee}$ is algebraic, so the conclusion follows from [61, Lemme 2.1].

Remark 4.2. It is not hard to see that $\mathcal{X}^{\vee}$ is a projective variety if and only if $\left(P, \mathcal{X}^{+}\right)$is pure. The argument is as follows: $\mathcal{X}^{\vee}$ is a holomorphic vector bundle over $\mathcal{X}_{G}^{\vee}$ where the fibre is homeomorphism to $W(\mathbb{R}) U(\mathbb{C})$. But $\mathcal{X}_{G}^{\vee}$ is projective, so $\mathcal{X}^{\vee}$ is projective if and only if it is a trivial vector bundle over $\mathcal{X}_{G}^{\vee}$, i.e. if and only if $W$ is trivial.

Let us take a closer look at the semi-algebraic structure of $\mathcal{X}^{+}$. By [67, pp 6], there exists a Shimura morphism $i:\left(G, \mathcal{X}_{G}^{+}\right) \rightarrow\left(P, \mathcal{X}^{+}\right)$s.t. $\pi \circ i=\mathrm{id}$. Then $i$ defines a Levi decomposition of $P=W \rtimes G$. By definition $\mathcal{X}^{+} \subset \operatorname{Hom}\left(\mathbb{S}_{\mathbb{C}}, P_{\mathbb{C}}\right)$. Define a bijective map

$$
\begin{aligned}
W(\mathbb{R}) U(\mathbb{C}) \times \mathcal{X}_{G}^{+} & \longrightarrow \mathcal{X}^{+} \\
(w, x) & \mapsto \operatorname{int}(w) \circ i(x)
\end{aligned} .
$$

Identify $P$ with the 4-tuple $(G, V, U, \Psi)$ as in $\S 2.2$. Since $W \simeq U \times V$ as $\mathbb{Q}$-varieties, we can define a bijection induced by the one above

$$
\rho: U(\mathbb{C}) \times V(\mathbb{R}) \times \mathcal{X}_{G}^{+} \stackrel{\sim}{\rightarrow} \mathcal{X}^{+}
$$

$P(\mathbb{R})^{+} U(\mathbb{C})$ acts on $\mathcal{X}^{+}$by definition. There is also a natural action of $P(\mathbb{R})^{+} U(\mathbb{C})$ on $U(\mathbb{C}) \times V(\mathbb{R}) \times \mathcal{X}_{G}^{+}$which is defined as follows. Under the notation of $\S 2.2$, for any $(u, v, g) \in$ $P(\mathbb{R})^{+} U(\mathbb{C})$ and $\left(u^{\prime}, v^{\prime}, x\right) \in U(\mathbb{C}) \times V(\mathbb{R}) \times \mathcal{X}_{G}^{+}$,

$$
(u, v, g) \cdot\left(u^{\prime}, v^{\prime}, x\right):=\left(u+g u^{\prime}+\frac{1}{2} \Psi\left(v, v^{\prime}\right), v+g v^{\prime}, g x\right) .
$$

This action is algebraic since $\Psi$ is a polynomial over $\mathbb{Q}$ (see $\S 2.2$ ). The morphism $\rho$ is $P(\mathbb{R})^{+} U(\mathbb{C})$ equivariant by an easy calculation.

Proposition 4.3. The map $\rho$ is semi-algebraic.

Proof. It is enough to prove that the graph of $\rho$ is semi-algebraic. This is true since $\rho$ is $P(\mathbb{R})^{+} U(\mathbb{C})$-equivariant and the actions of $P(\mathbb{R})^{+} U(\mathbb{C})$ on both sides are algebraic and transitive. Explicitly, fix a point $x_{0} \in U(\mathbb{C}) \times V(\mathbb{R}) \times \mathcal{X}_{G}^{+}$, the graph of $\rho$

$$
\begin{aligned}
G r(\rho) & =\left\{\left(g x_{0}, \rho\left(g x_{0}\right)\right) \in\left(U(\mathbb{C}) \times V(\mathbb{R}) \times \mathcal{X}_{G}^{+}\right) \times \mathcal{X}^{+} \mid g \in P(\mathbb{R})^{+} U(\mathbb{C})\right\} & & \text { (transitivity) } \\
& =\left\{\left(g x_{0}, g \rho\left(x_{0}\right)\right) \in\left(U(\mathbb{C}) \times V(\mathbb{R}) \times \mathcal{X}_{G}^{+}\right) \times \mathcal{X}^{+} \mid g \in P(\mathbb{R})^{+} U(\mathbb{C})\right\} & & \text { (equivariance) } \\
& =P(\mathbb{R})^{+} U(\mathbb{C}) \cdot\left(x_{0}, \rho\left(x_{0}\right)\right) & &
\end{aligned}
$$


is semi-algebraic since the action of $P(\mathbb{R})^{+} U(\mathbb{C})$ on $\left(U(\mathbb{C}) \times V(\mathbb{R}) \times \mathcal{X}_{G}^{+}\right) \times \mathcal{X}^{+}$is algebraic.

Remark 4.4. If $U$ is trivial, then the complex structure of $\mathcal{X}^{+}$given by $\mathcal{X}^{\vee}$ is the same as the one given by [54, Construction 2.9] since for the projection $\mathcal{X}^{+} \stackrel{\pi}{\rightarrow} \mathcal{X}_{G}^{+}$, the complex structure of any fibre $\mathcal{X}_{x_{G}}^{+}\left(x_{G} \in \mathcal{X}_{G}^{+}\right)$given by $\mathcal{X}^{\vee}$ is the same as the one obtained from $\mathcal{X}_{x_{G}}^{+} \simeq V(\mathbb{C}) / F_{x_{G}}^{0} V(\mathbb{C})$ $($ see $[53,3.13,3.14])$. In particular this holds for $\mathcal{X}_{2 g, a}^{+}$(see $§ 2.3$ for notation). Therefore for any $\mathfrak{A}_{g}(N)$, the fundamental set $[0, N)^{2 g} \times \mathcal{F}_{G} \subset V_{2 g}(\mathbb{R}) \times \mathbb{H}_{g}^{+} \simeq \mathcal{X}_{2 g, a}^{+}$is the one considered in $[45]$.

\section{5. (WEAKLY) SPECIAL SUBVARIETIES}

\subsection{Weakly special subvarieties.}

Definition 5.1. (Pink, [54, Definition 4.1(b)]) Let $S$ be a connected mixed Shimura variety. Consider any Shimura morphisms $T^{\prime} \stackrel{[\varphi]}{\longleftarrow} T \stackrel{[i]}{\longrightarrow} S$ and any point $t^{\prime} \in T^{\prime}$. Then any irreducible component of $[i]\left([\varphi]^{-1}\left(t^{\prime}\right)\right)$ is called a weakly special subvariety of $S$. We will prove later in Remark 5.5 that weakly special subvarieties of $S$ are indeed closed subvarieties.

Since any Shimura morphism is related to a Shimura morphism between Shimura data, we will try to rephrase this definition in the context of Shimura data:

Definition 5.2. Given a connected mixed Shimura datum $\left(P, \mathcal{X}^{+}\right)$, a weakly special subset of $\mathcal{X}^{+}$is a connected component of $i\left(\varphi^{-1}\left(y^{\prime}\right)\right) \subset \mathcal{X}^{+}$for a point $y^{\prime} \in \mathcal{Y}^{\prime+}$, where $i, \varphi, \mathcal{Y}^{\prime+}$ are in the following diagram of Shimura morphisms

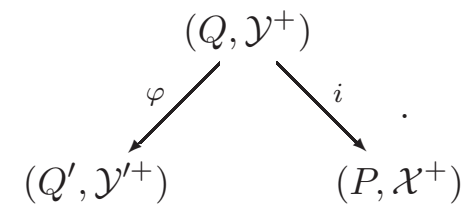

Remark 5.3. (1) In the definition above, let $N:=\operatorname{Ker}\left(Q \rightarrow Q^{\prime}\right)$ and let $U_{N}:=U_{Q} \cap$ $N$, then $i\left(\varphi^{-1}\left(y^{\prime}\right)\right)$ is a connected component of $N(\mathbb{R}) U_{N}(\mathbb{C}) y$ where $\varphi(y)=y^{\prime}$. So $i\left(\varphi^{-1}\left(y^{\prime}\right)\right)$ is smooth as an analytic variety. In particular, its connected components and complex analytic irreducible components coincide. As a result, we can replace " $a$ connected component" by "a complex analytic irreducible component" in Definition 5.2.

(2) If furthermore $N$ is connected, then $i\left(\varphi^{-1}\left(y^{\prime}\right)\right)$ itself is connected (hence also complex analytic irreducible). The proof is as follows: Consider the image of $\varphi^{-1}\left(y^{\prime}\right)$ under the projection $\left(Q, \mathcal{Y}^{+}\right) \stackrel{\pi}{\rightarrow}\left(G_{Q}, \mathcal{Y}_{G_{Q}}^{+}\right):=\left(Q, \mathcal{Y}^{+}\right) / W_{Q}$. By the decomposition ([40, 3.6])

$$
\left(G_{Q}^{\mathrm{ad}}, \mathcal{Y}_{G_{Q}}^{+}\right)=\left(G_{N}^{\mathrm{ad}}, \mathcal{Y}_{1}^{+}\right) \times\left(G_{2}, \mathcal{Y}_{2}^{+}\right)
$$

where $G_{N}:=N / W \cap N, \pi\left(\varphi^{-1}\left(y^{\prime}\right)\right)=\mathcal{Y}_{1}^{+} \times\left\{y_{2}\right\}$. So $\pi\left(\varphi^{-1}\left(y^{\prime}\right)\right)=G_{N}(\mathbb{R})^{+} \pi(y)$. But $W_{N}(\mathbb{R}) U_{N}(\mathbb{C})\left(W_{N}:=W \cap N\right)$ is connected, hence $\varphi^{-1}\left(y^{\prime}\right)=N(\mathbb{R})^{+} U_{N}(\mathbb{C}) y$, which is connected. In consequence, $i\left(\varphi^{-1}\left(y^{\prime}\right)\right)$ also is connected.

Proposition 5.4. For any weakly special subvariety of $S$ (resp. weakly special subset of $\mathcal{X}^{+}$), the Shimura morphisms in Definition 5.1 (resp. Definition 5.2) can be chosen such that

- the underlying homomorphism of algebraic groups $i$ is injective, and hence $i$ is an embedding in the sense of [53, 2.3]; 
- the underlying homomorphism of algebraic groups $\varphi$ is surjective, and its kernel $N$ is connected. Moreover, $N$ possesses no non-trivial torus quotient (or equivalently, $G_{N}:=$ $N /(W \cap N)$ is semi-simple);

- $\varphi$ is a quotient Shimura morphism.

Proof. If $P=\operatorname{MT}\left(\mathcal{X}^{+}\right)$, then the first two points except the statement in the bracket are proved by [54, Proposition 4.4]. The general case follows directly from Proposition 2.6(1). The third assertion can be proved by the universal property of quotient Shimura data $([53,2.9])$. Now we are left to prove the statement in the bracket.

$G_{N} \triangleleft G$ since $G_{N}=N /(W \cap N) \hookrightarrow G=P / W$ and $N \triangleleft P$, and hence $G_{N}$ is reductive $\left(\left[14,14.2\right.\right.$, Corollary(b)]). By [14, 14.2 Proposition(2)], $G_{N}$ is the almost-product of $G_{N}^{\text {der }}$ and $Z\left(G_{N}\right)^{\circ}$, and $Z\left(G_{N}\right)^{\circ}$ equals the radical of $G_{N}$ which is a torus. So $N$ possesses no non-trivial torus quotient iff $G_{N}$ possesses no non-trivial torus quotient iff $G_{N}$ is semi-simple.

Remark 5.5. We can now prove that weakly special subvarieties of $S$ are closed. By the proposition above, we can choose $i$ to be injective. Then $[i]$ is finite by $[53,3.8]$. Hence $[i]\left([\varphi]^{-1}\left(t^{\prime}\right)\right)$ is closed.

Lemma 5.6. Suppose that the Shimura morphisms $T^{\prime} \stackrel{[\varphi]}{\longleftarrow} T \stackrel{[i]}{\longrightarrow} S$ are associated with the morphisms of mixed Shimura data

$$
\left(Q^{\prime}, \mathcal{Y}^{\prime+}\right) \stackrel{\varphi}{\leftarrow}\left(Q, \mathcal{Y}^{+}\right) \stackrel{i}{\rightarrow}\left(P, \mathcal{X}^{+}\right)
$$

so that we have the following commutative diagram

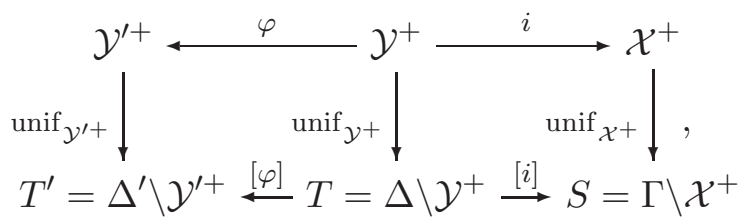

then for any point $y^{\prime} \in \mathcal{Y}^{\prime+}$, any irreducible component of unif $\mathcal{X}_{+}\left(i\left(\varphi^{-1}\left(y^{\prime}\right)\right)\right)$ is also an irreducible component of $[i]\left([\varphi]^{-1}\left(\operatorname{unif}_{\mathcal{Y}^{\prime+}}\left(y^{\prime}\right)\right)\right)$.

Proof. Let $N:=\operatorname{Ker}(\varphi)$ and let $U_{Q}$ be the weight -2 part of $Q$, then we have

$$
\operatorname{unif}_{\mathcal{X}^{+}}\left(i\left(\varphi^{-1}\left(y^{\prime}\right)\right)\right) \subset[i]\left([\varphi]^{-1}\left(\operatorname{unif}_{\mathcal{Y}^{+}}\left(y^{\prime}\right)\right)\right),
$$

and both of them are of constant dimension $d$, where $d$ is the dimension of any orbit of $N(\mathbb{R})^{+}\left(U_{Q} \cap N\right)(\mathbb{C})$. This allows us to conclude.

The following Proposition tells us that the two definitions of weak specialness are compatible.

Proposition 5.7. Let $S$ be a connected mixed Shimura variety associated with the connected mixed Shimura datum $\left(P, \mathcal{X}^{+}\right)$and let unif: $\mathcal{X}^{+} \rightarrow S=\Gamma \backslash \mathcal{X}^{+}$be the uniformization, then a subvariety $Z$ of $S$ is weakly special if and only if $Z$ is the image of some weakly special subset of $\mathcal{X}^{+}$.

Proof. The "if" part is immediate by Lemma 5.6. We prove the "only if" part. We assume that $i, \varphi$ are as in Proposition 5.4. For any weakly special subvariety $Z \subset S$, suppose that we have 
a diagram as in Lemma 5.6 and $Z$ is an irreducible component of $[i]\left([\varphi]^{-1}\left(t^{\prime}\right)\right)$. Since

$$
[i]\left([\varphi]^{-1}\left(t^{\prime}\right)\right) \subset \bigcup_{y^{\prime} \in \operatorname{unif}_{\mathcal{Y}^{+}}^{-1}\left(t^{\prime}\right)} \operatorname{unif}_{\mathcal{X}^{+}}\left(i\left(\varphi^{-1}\left(y^{\prime}\right)\right)\right)=\operatorname{unif}_{\mathcal{X}^{+}}\left(i\left(\varphi^{-1}\left(\operatorname{unif}_{\mathcal{Y}^{+}}^{-1}\left(t^{\prime}\right)\right)\right)\right),
$$

there exists a $y^{\prime} \in \mathcal{Y}^{\prime+}$ lying over $t^{\prime}$ s.t. $Z$ is an irreducible component of unif $\mathcal{X}^{+}\left(i\left(\varphi^{-1}\left(y^{\prime}\right)\right)\right)$ by Lemma 5.6. By Remark 5.3.2, $i\left(\varphi^{-1}\left(y^{\prime}\right)\right)$ is complex analytic irreducible, so unif $\mathcal{X}^{+}\left(i\left(\varphi^{-1}\left(y^{\prime}\right)\right)\right)$ is also complex analytic irreducible when $S$ is regarded as an analytic variety. Hence unif $\mathcal{X}^{+}\left(i\left(\varphi^{-1}\left(y^{\prime}\right)\right)\right)$ is irreducible as an algebraic variety. So $Z=\operatorname{unif}_{\mathcal{X}^{+}}\left(i\left(\varphi^{-1}\left(y^{\prime}\right)\right)\right)$.

We close this subsection by proving that this definition of weakly special subvarieties is compatible with the one (which is already known) for pure Shimura varieties.

Proposition 5.8. A weakly special subvariety of a pure Shimura variety $S$ is a subvariety of the same form as in [62, Definition 2.1].

Proof. This is pointed out in [54, Remark 4.5]. We give a (relatively) detailed proof here. We prove the result for weakly special subsets. Assume that $S$ is associated with the connected pure Shimura datum $\left(P, \mathcal{X}^{+}\right)$. For a subset of the same form as in [62, Definition 2.1], take $\left(Q, \mathcal{Y}^{+}\right)=\left(H, X_{H}^{+}\right)$and $\left(Q^{\prime}, \mathcal{Y}^{\prime+}\right)=\left(H_{1}, X_{1}^{+}\right)$(same notation as [62, Definition 2.1]). Then by definition such a subset is weakly special (as in Definition 5.2).

On the other hand, suppose that we have a weakly special subset $\widetilde{F}$ defined by a diagram as in Definition 5.2 satisfying Proposition 5.4. Let $N:=\operatorname{Ker}(\varphi)$, then the homogeneous spaces of the connected pure Shimura data $\left(Q^{\prime}, \mathcal{Y}^{\prime+}\right)=\left(Q, \mathcal{Y}^{+}\right) / N$ and $\left(Q, \mathcal{Y}^{+}\right) / Z(Q) N=\left(Q^{\text {ad }}, \mathcal{Y}^{\text {ad }+}\right) / N^{\text {ad }}$ are canonically isomorphic to each other $\left(\left[39\right.\right.$, Proposition 5.7]). Hence we may replace $\left(Q^{\prime}, \mathcal{Y}^{\prime+}\right)$ by $\left(Q^{\mathrm{ad}}, \mathcal{Y}^{\mathrm{ad}+}\right) / N^{\mathrm{ad}}$. But by $[40,3.6,3.7],\left(Q^{\mathrm{ad}}, \mathcal{Y}^{\mathrm{ad}+}\right)=\left(N^{\mathrm{ad}}, \mathcal{Y}_{1}^{+}\right) \times\left(Q_{2}, \mathcal{Y}_{2}^{+}\right)$. So $\widetilde{F}$ is of the same form as in [62, Definition 2.1].

\subsection{Special subvarieties.}

Definition 5.9. Let $S$ be a connected mixed Shimura variety associated with the connected mixed Shimura datum $\left(P, \mathcal{X}^{+}\right)$.

(1) A special subvariety of $S$ is the image of any Shimura morphism $T \rightarrow S$ of connected mixed Shimura varieties;

(2) A point $x \in \mathcal{X}^{+}$and its image in $S$ are called special if the homomorphism $x: \mathbb{S}_{\mathbb{C}} \rightarrow P_{\mathbb{C}}$ factors through $T_{\mathbb{C}}$ for a torus $T \subset P$.

Remark 5.10. By definition, $x \in \mathcal{X}^{+}$is special if and only if it is the image of a Shimura morphism $\left(T, \mathcal{Y}^{+}\right) \hookrightarrow\left(P, \mathcal{X}^{+}\right)$. Hence a special point is just a special subvariety of dimension 0 .

The following result is easy to prove.

Lemma 5.11. Let $S$ be a connected mixed Shimura variety associated with the connected mixed Shimura datum $\left(P, \mathcal{X}^{+}\right)$and let unif: $\mathcal{X}^{+} \rightarrow S$ be the uniformizing map, then a subvariety of $S$ is special if and only if it is of the form unif $\left(\mathcal{Y}^{+}\right)$for some $\left(Q, \mathcal{Y}^{+}\right) \hookrightarrow\left(P, \mathcal{X}^{+}\right)$.

Proposition 5.12. Every special subvariety of $S$ contains a Zariski dense subset of special points.

Proof. [54, Proposition 4.14]. 
The relation between special and weakly special subvarieties is:

Proposition 5.13. A subvariety of $S$ is special if and only if it is weakly special and contains one special point.

Proof. [54, Proposition 4.2, Proposition 4.14, Proposition 4.15].

\section{Algebraicity in the Uniformizing SPACE}

Definition 6.1. Let $\widetilde{Y}$ be an analytic subset of $\mathcal{X}^{+}$, then

(1) $\tilde{Y}$ is called an irreducible algebraic subset of $\mathcal{X}^{+}$if it is a complex analytic irreducible component of the intersection of its Zariski closure in $\mathcal{X}^{\vee}$ and $\mathcal{X}^{+}$;

(2) $\tilde{Y}$ is called algebraic if it is a finite union of irreducible algebraic subsets of $\mathcal{X}^{+}$.

Lemma 6.2. Any weakly special subset of $\mathcal{X}^{+}$is irreducible algebraic.

Proof. Suppose that $\widetilde{Z}$ is a weakly special subset of $\mathcal{X}^{+}$. Use the notation of Definition 5.2 and assume that $i$ and $\varphi$ satisfy the properties in Proposition 5.4. Let $N:=\operatorname{Ker}\left(Q \rightarrow Q^{\prime}\right)$ and let $y$ be a point of the weakly special subset, then $\widetilde{Z}=N(\mathbb{R})^{+} U_{N}(\mathbb{C}) y$ is complex analytic irreducible by Remark 5.3.2. But $N(\mathbb{R})^{+} U_{N}(\mathbb{C}) y=N(\mathbb{C}) y \cap \mathcal{X}^{+}$and $N(\mathbb{C}) y$ is algebraic, so $\widetilde{Z}$ is irreducible algebraic by definition.

Lemma 6.3 (functoriality of algebraicity). Let $f:\left(Q, \mathcal{Y}^{+}\right) \rightarrow\left(P, \mathcal{X}^{+}\right)$be a Shimura morphism. Then there exists a unique morphism $f^{\vee}: \mathcal{Y}^{\vee} \rightarrow \mathcal{X}^{\vee}$ of algebraic varieties such that the diagram commutes:

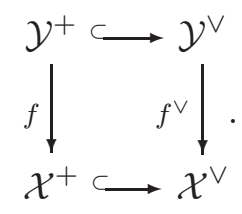

Furthermore, for any irreducible algebraic subset $\widetilde{Z}$ of $\mathcal{Y}^{+}$, the closure w.r.t the archimedean topology of $f(\widetilde{Z})$ is irreducible algebraic in $\mathcal{X}^{+}$and $f(\widetilde{Z})$ contains a dense open subset of this closure.

In particular, if $f$ is an embedding, then an irreducible algebraic subset of $\mathcal{Y}^{+}$is an irreducible component of the intersection of an irreducible algebraic subset of $\mathcal{X}^{+}$with $\mathcal{Y}^{+}$.

Proof. Fix a point $x_{0} \in \mathcal{Y}^{+}$, then we have

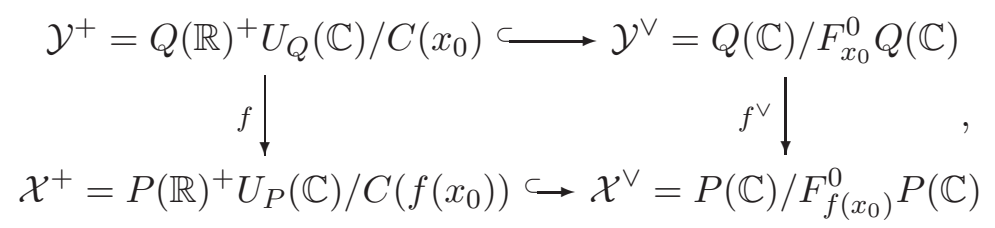

where $C\left(x_{0}\right)$ (resp. $C\left(f\left(x_{0}\right)\right)$ ) denotes the stabilizer of $x_{0}$ (resp. $\left.f\left(x_{0}\right)\right)$ in $Q(\mathbb{R}) U_{Q}(\mathbb{C})$ (resp. $\left.P(\mathbb{R}) U_{P}(\mathbb{C})\right) . f^{\vee}$ is unique since $Q(\mathbb{R}) U_{Q}(\mathbb{C}) / C\left(x_{0}\right)$ is dense in $\mathcal{Y}^{\vee}$.

To prove the second statement, it is enough to prove the result for $f^{\vee}\left(\overline{\widetilde{Z}}^{\text {Zar }}\right) \subset \mathcal{X}^{\vee}$ where $\overline{\widetilde{Z}}^{\text {Zar }}$ is the Zariski closure of $\widetilde{Z}$ in $\mathcal{Y}^{\vee}$. This is then an algebro-geometric result, which follows easily from Chevalley's Theorem ([29, Chapitre IV, 1.8.4]) and [41, I.10, Theorem 1]. 


\section{Results FOR The UNIPOTENT PART}

Given a connected mixed Shimura variety $S$, let $S_{G}$ be its pure part so that we have a projection $S \rightarrow S_{G}$. For any point $b \in S_{G}$, denote by $E$ the fiber $S_{b}$. Suppose that $S$ is associated with the mixed Shimura datum $\left(P, \mathcal{X}^{+}\right)$, which can be further assumed to be irreducible by Proposition 2.6. Let unif: $\mathcal{X}^{+} \rightarrow S=\Gamma \backslash \mathcal{X}^{+}$be the uniformization. Now $E=S_{b} \simeq \Gamma_{W} \backslash W(\mathbb{R}) U(\mathbb{C})$ with the complex structure determined by $b \in S_{G}\left(E=S_{b}=\Gamma_{W} \backslash W(\mathbb{C}) / F_{b}^{0} W(\mathbb{C})\right)$, where $\Gamma_{W}:=\Gamma \cap W(\mathbb{Q})$. Write $T:=\Gamma_{U} \backslash U(\mathbb{C})$ and $A:=\Gamma_{A} \backslash V(\mathbb{C}) / F_{b}^{0} V(\mathbb{C})$ where $\Gamma_{U}:=\Gamma \cap U(\mathbb{Q})$ and $\Gamma_{V}:=\Gamma_{W} / \Gamma_{U}$, then $T$ is an algebraic torus over $\mathbb{C}, A$ is a complex abelian variety and $E$ is an algebraic torus over $A$ whose fibers are isomorphic to $T$.

Lemma 7.1. If $E$ can be given the structure of an algebraic group whose group law is compatible with that of $W$, then $W$ (hence $E$ ) is commutative. In this case $E$ is a semi-abelian variety.

Proof. If $E$ is an algebraic group, then $T$ is a normal subgroup of $E$. Hence $E$ acts on $T$ by conjugation, and this action factors via $A$, and then it is trivial by [14, 8.10 Proposition]. Therefore $T$ is in the center of $E$. Now consider the commutator pairing $E \times E \rightarrow E$. This factors through a morphism $A \times A \stackrel{f}{\rightarrow} T$. But this morphism is then constant. So the commutator pairing $E \times E \rightarrow E$ is trivial, and hence $E$ is commutative.

The commutator pairing $W \times W \rightarrow W$ induces an alternating form $\Psi: V \times V \rightarrow U$ (same as $\$ 2.2$ ) which induces the morphism $f$ above. We have proved in the last paragraph that $\Psi(V(\mathbb{R}), V(\mathbb{R})) \subset \Gamma_{U}$ with $\Gamma_{U}:=\Gamma \cap U(\mathbb{Q})$. But $\Psi(V(\mathbb{R}), v)$ is continuous for any $v \in V(\mathbb{R})$ and $\Psi(0, V(\mathbb{R}))=0$, so $\Psi(V(\mathbb{R}), V(\mathbb{R}))=0$. Hence the commutator pairing $W \times W \rightarrow W$ is trivial, and therefore $W$ is commutative.

\subsection{Weakly special subvarieties of a complex semi-abelian variety.}

Proposition 7.2. Use the notation as at the beginning of the section. Weakly special subvarieties of $E$ are precisely the subsets of $E$ of the form

$$
\operatorname{unif}\left(W_{0}(\mathbb{R}) U_{0}(\mathbb{C}) \widetilde{z}\right)
$$

where $W_{0}$ is a $\mathrm{MT}(b)$-subgroup of $W$ (i.e. a subgroup of $W$ normalized by $\left.\mathrm{MT}(b)\right), U_{0}:=W_{0} \cap U$, $\operatorname{unif}\left(\widetilde{z}_{G}\right)=b$ and $\widetilde{z}_{V} \in\left(N_{W}\left(W_{0}\right) / U\right)(\mathbb{R})\left(\widetilde{z}=\left(\widetilde{z}_{U}, \widetilde{z}_{V}, \widetilde{z}_{G}\right)\right.$ under (4.1)).

In particular, if $E$ can be given the structure of an algebraic group whose group law is compatible with that of $W$ (i.e. $W$ is commutative), then the weakly special subvarieties of $E$ are precisely the translates of subgroups of $E$.

Proof. Let $Z$ be a weakly special variety of $E$ and let $\widetilde{Z}$ be a complex analytic irreducible component of unif $^{-1}(Z)$, then there exists a diagram as in Definition 5.2 s.t. $\widetilde{z}: \mathbb{S}_{\mathbb{C}} \rightarrow P_{\mathbb{C}}$ factors through $Q_{\mathbb{C}}, N \triangleleft Q$ and $\widetilde{Z}=N(\mathbb{R})^{+} U_{N}(\mathbb{C}) \widetilde{z}$ for some $\widetilde{z} \in \widetilde{Z}$. As is explained in [54, paragraph 2, pp 265], $G_{N}=1$. We prove that $N=W_{N}$ satisfies the conditions which we require. Let $U_{N}:=W_{N} \cap U$, then $U_{N}$ is a $\mathrm{MT}(b)$-module by Proposition 2.6(2). Denote by $V_{N}:=W_{N} / U_{N}, \pi_{P / U}:\left(P, \mathcal{X}^{+}\right) \rightarrow\left(P / U, \mathcal{X}_{P / U}^{+}\right)$and $\left[\pi_{P / U}\right]: S \rightarrow S_{P / U}$. Then $\left[\pi_{P / U}\right](Z)$ is a

subvariety of $A$ since $Z$ is a subvariety of $E$. So $\pi_{P / U}(\widetilde{Z})=V_{N}(\mathbb{R})+\pi_{P / U}(\widetilde{z})$ is translate of a complex subspace of $V(\mathbb{R})=V(\mathbb{C}) / F_{b}^{0} V(\mathbb{C})$, and therefore $V_{N}$ is a $\mathrm{MT}(b)$-module. So $W_{N}$ is stable under the action of $\operatorname{MT}(b)$. Now $\widetilde{z}_{V} \in\left(N_{W}(N) / U\right)(\mathbb{R})$ since $\widetilde{z}: \mathbb{S}_{\mathbb{C}} \rightarrow P_{\mathbb{C}}$ factors through $N_{P}(N)_{\mathbb{C}}$ 
Conversely let $\widetilde{Z}=W_{0}(\mathbb{R}) U_{0}(\mathbb{C}) \widetilde{z}$ with $W_{0}, \widetilde{z}$ as stated. Fix a Levi decomposition $P=W \rtimes G$. Let $G^{\prime}:=\operatorname{MT}(b)$, let $W^{\prime}:=N_{W}\left(W_{0}\right)$ and define $Q:=W^{\prime} \rtimes G^{\prime}$. By definition of $Q, W_{0} \triangleleft Q$ and there exists a connected mixed Shimura datum $\left(Q, \mathcal{Y}^{+}\right) \hookrightarrow\left(P, \mathcal{X}^{+}\right)$with $b \in \operatorname{unif}\left(\mathcal{Y}^{+}\right)$. Now consider the morphisms of connected mixed Shimura data

$$
\left(Q, \mathcal{Y}^{+}\right) / W_{0} \stackrel{\varphi}{\leftarrow}\left(Q, \mathcal{Y}^{+}\right) \stackrel{i}{\rightarrow}\left(P, \mathcal{X}^{+}\right) .
$$

In the fibres above the point $b \in S_{G}$ these maps are simply

$$
S_{Q, b} / Z \nleftarrow S_{Q, b} \hookrightarrow E=S_{b} .
$$

Hence $Z$ is a weakly special subvariety by definition.

Corollary 7.3. (1) Weakly special subvarieties of a complex abelian variety are precisely the translates of abelian subvarieties;

(2) Weakly special subvarieties of an algebraic torus over $\mathbb{C}$ are precisely the translates of subtori.

Proof. This is a direct consequence of Proposition 7.2.

7.2. Smallest weakly special subvariety containing a given subvariety of an abelian variety or an algebraic torus over $\mathbb{C}$.

Proposition 7.4. (1) Let $X$ be a complex abelian variety and let $Z$ be a closed irreducible subvariety of $X$. Denote by

$$
\widetilde{X}=\pi_{1}(X, z) \otimes_{\mathbb{Z}} \mathbb{R}=H_{1}(X, \mathbb{R}) \simeq \mathbb{C}^{n} \stackrel{u}{\rightarrow} X
$$

the universal cover of $X\left(z \in Z^{\mathrm{sm}}\right)$, then the smallest weakly special subvariety of $X$ containing $Z$ is a translate of $u\left(\pi_{1}\left(Z^{\mathrm{sm}}, z\right) \otimes \mathbb{R}\right)$.

(2) Let $X$ be an algebraic torus over $\mathbb{C}$ and let $Z$ be a closed irreducible subvariety of $X$. Denote by

$$
\widetilde{X}=\pi_{1}(X, z) \otimes_{\mathbb{Z}} \mathbb{C}=H_{1}(X, \mathbb{C}) \simeq \mathbb{C}^{n} \stackrel{u}{\rightarrow} X
$$

the universal cover of $X\left(z \in Z^{\mathrm{sm}}\right)$, then the smallest weakly special subvariety of $X$ containing $Z$ is a translate of $u\left(\pi_{1}\left(Z^{\mathrm{sm}}, z\right) \otimes \mathbb{C}\right)$.

Proof. (1) If $X$ is a complex abelian variety, then the result is due to Ullmo-Yafaev. Their proof of [62, Proposition 5.1] has in fact revealed this property. Here we restate the proof with more details.

Let $Z^{\text {de }} \stackrel{s}{\rightarrow} Z$ be a desingularization of $Z^{\text {de }}$ s.t. there exists a Zariski open subset $Z_{0}^{\text {de }}$ of $Z^{\text {de }}$ s.t. $Z_{0}^{\text {de }} \underset{s}{\stackrel{\sim}{\rightarrow}} Z^{\text {sm }}$. By the commutative diagram

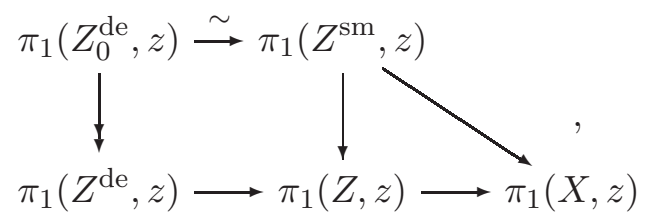

where $z \in Z^{\mathrm{sm}}$ (the surjectivity on the left is due to $[37,2.10 .1]$ ), we know that the image of $\pi_{1}\left(Z^{\mathrm{de}}, z\right)$ and the image of $\pi_{1}\left(Z^{\mathrm{sm}}, z\right)$ in $\pi_{1}(X, z)$ are the same. 
Let $\operatorname{Alb}\left(Z^{\text {de }}\right)$ be the Albanese variety of $Z^{\text {de }}$ normalized by $z$, then the map $\tau: Z^{\text {de }} \rightarrow$ $Z \rightarrow X$ factors uniquely through the Albanese morphism([66, Theorem 12.15]):

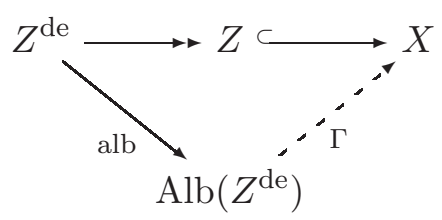

Let $A:=\Gamma\left(\operatorname{Alb}\left(Z^{\text {de }}\right)\right)$, then it is the smallest weakly special subvariety (i.e. the translate of an abelian subvariety) of $X$ containing $Z$ since $\operatorname{alb}\left(Z^{\text {de }}\right)$ generates $\operatorname{Alb}\left(Z^{\text {de }}\right)$ ([66, Lemma 12.11]).

It suffices to prove that the image of $\pi_{1}\left(Z^{\mathrm{de}}, z\right)$ in $\pi_{1}(X, z) \simeq H_{1}(X, \mathbb{Z})$ is of finite index in $H_{1}(A, \mathbb{Z})$. This is true since the image of $\pi_{1}\left(Z^{\mathrm{de}}, z\right)$ in $H_{1}(X, \mathbb{Z})$ contains

$$
(\Gamma \circ \mathrm{alb})_{*} H_{1}\left(Z^{\mathrm{de}}, \mathbb{Z}\right) \simeq \Gamma_{*} H_{1}\left(\operatorname{Alb}\left(Z^{\mathrm{de}}\right), \mathbb{Z}\right) \simeq \Gamma_{*} \pi_{1}\left(\operatorname{Alb}\left(Z^{\mathrm{de}}\right)\right)
$$

(the first isomorphism is given by the definition of Albanese varieties via Hodge theory, see e.g. the proof of [66, Lemma 12.11]), which is of finite index in $\pi_{1}(A, z) \simeq H_{1}(A, \mathbb{Z})$ by $[37,2.10 .2]$.

(2) If $X$ is an algebraic torus over $\mathbb{C}$, then we can first of all translate $Z$ by a point s.t. the translate contains the origin of $X$. Now we are done if we can prove that the smallest subtorus containing this translate of $Z$ is $u\left(\pi_{1}\left(Z^{\mathrm{sm}}, z\right) \otimes_{\mathbb{Z}} \mathbb{C}\right)$.

Suppose $T \simeq\left(\mathbb{C}^{*}\right)^{m}$ is the smallest sub-torus of $X$ containing $Z$ with $j: Z^{\mathrm{sm}} \hookrightarrow T$ the inclusion. We are done if we can prove $\left[\pi_{1}(T, z): j_{*} \pi_{1}\left(Z^{\mathrm{sm}}, z\right)\right]<\infty$. If not, then

$$
j_{*} \pi_{1}\left(Z^{\mathrm{sm}}, z\right) \subset \operatorname{Ker}\left(\mathbb{Z}^{m} \stackrel{\rho}{\rightarrow} \mathbb{Z}\right)
$$

for some map $\rho$. Since the covariant functor $T \mapsto X_{*}(T)\left(X_{*}(T)\right.$ is the co-character group of $T$ ) is an equivalence between the category algebraic tori over $\mathbb{C}$ and their morphisms as algebraic groups $\}$ and the category $\{$ free $\mathbb{Z}$-modules of finite rank $\}$, the map $\rho$ corresponds to a surjective map (with connected kernel) of tori $p: T \rightarrow T^{\prime}$. The composition of the maps $Z^{\mathrm{sm}} \stackrel{j}{\rightarrow} T \stackrel{p}{\rightarrow} T^{\prime}=\mathbb{G}_{m, \mathbb{C}}$ should be dominant by the choice of $T$. But then we have

$$
\left[\pi_{1}\left(T^{\prime}, p(z)\right):(p \circ j)_{*} \pi_{1}\left(Z^{\mathrm{sm}}, z\right)\right]<\infty
$$

$([37,2.10 .2])$, which contradicts $(7.1)$ by the following lemma.

Lemma 7.5. For any $\mathbb{C}$-split torus $T \simeq\left(\mathbb{C}^{*}\right)^{n}$, we have a canonical isomorphism

$$
X_{*}(T) \stackrel{\psi_{T}}{\sim} \pi_{1}(T, 1) .
$$

Here "canonical" means that for any morphism (between algebraic groups) $f: T \rightarrow T^{\prime}$ between two such $\mathbb{C}$-split tori, the following diagram commutes:

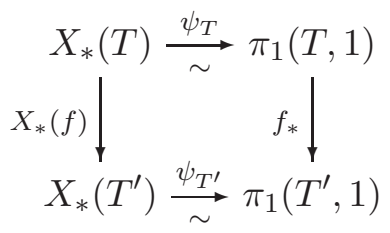


Proof. Denote by $U_{1}:=\{z \in \mathbb{C}|| z \mid=1\}$ and $i: U_{1} \hookrightarrow \mathbb{C}^{*}$ the inclusion. Then the map $\psi_{T}$ is defined by

$$
\begin{gathered}
X_{*}(T) \stackrel{\psi_{T}}{\longrightarrow} \pi_{1}(T, 1) \\
\nu \quad \mapsto[\nu \circ i]
\end{gathered} .
$$

This is clearly a group homomorphism. It is surjective since a representative of the generators of $\pi_{1}(T, 1)$ is given by the $n$ coordinate embeddings $U_{1} \hookrightarrow \mathbb{C}^{*} \hookrightarrow T=\left(\mathbb{C}^{*}\right)^{n}$. $\psi_{T}$ is injective since $X_{*}(T) \simeq \pi_{1}(T, 1) \simeq \mathbb{Z}^{n}$ is torsion-free. The rest of the lemma is immediate by the construction of $\psi_{T}$.

7.3. Ax-Lindemann-Weierstraß for the unipotent part. By abuse of notation we denote the uniformization of $E$ by unif: $W(\mathbb{R}) U(\mathbb{C})=W(\mathbb{C}) / F_{b}^{0} W(\mathbb{C}) \rightarrow E$. It is then the restriction of unif : $\mathcal{X}^{+} \rightarrow S$.

Theorem 7.6. Let $Y$ be a closed irreducible subvariety of $E$ and let $\widetilde{Z}$ be a maximal irreducible algebraic subvariety which is contained in unif $^{-1}(Y)$. Then $\widetilde{Z}$ is weakly special.

Proof. If $E$ is an algebraic torus over $\mathbb{C}$, this is a consequence of the Ax-Schanuel theorem [42, Corollary 3.6]. If $E$ is an abelian variety, this is Pila-Zannier [52, pp9, Remark 1]. A proof using volume calculation and points counting method for these two cases can be found in the Appendix. The general case will be proved in $\S 11$.

\section{The SMAllest WEAKLY SPECIAL SUbVARIETy CONTAINING A GIVEN SUBVARIETY}

In this section, our goal is to prove a theorem (Theorem 8.1) which (in some sense) generalizes $[40,3.6,3.7]$. In particular, we get a criterion of weak specialness as a corollary (Corollary 8.3) which generalizes [62, Theorem 4.1]. Before the proof, let us do some technical preparation at first.

Let $S$ be a connected mixed Shimura variety associated with the connected mixed Shimura datum $\left(P, \mathcal{X}^{+}\right)$and let unif: $\mathcal{X}^{+} \rightarrow S=\Gamma \backslash \mathcal{X}^{+}$be the uniformization. We may assume $P=$ $\operatorname{MT}\left(\mathcal{X}^{+}\right)$by Proposition 2.6. There exists a $\Gamma^{\prime} \leqslant \Gamma$ of finite index s.t. $\Gamma^{\prime}$ is neat. Let $S^{\prime}:=$ $\Gamma^{\prime} \backslash \mathcal{X}^{+}$and let unif': $\mathcal{X}^{+} \rightarrow S^{\prime}$ be its uniformization. Choose any faithful $\mathbb{Q}$-representation $\xi: P \rightarrow \mathrm{GL}(M)$ of $P$, then Theorem 3.7 claims that $\xi$ (together with a choice of a $\Gamma^{\prime}$-invariant lattice of $M$ ) gives rise to an admissible variation of mixed Hodge structure on $S^{\prime}$. The generic Mumford-Tate group of this variation is $P$.

Suppose that $Y$ is a closed irreducible subvariety of $S$. Let $Y^{\prime}$ be an irreducible component of $p^{-1}(Y)$ under $p: S^{\prime}=\Gamma^{\prime} \backslash \mathcal{X}^{+} \rightarrow S=\Gamma \backslash \mathcal{X}^{+}$, then $Y^{\prime}$ is a closed irreducible subvariety of $S^{\prime}$ which maps surjectively to $Y$ under $p$. The variation we constructed above can be restricted to $Y^{\prime s m}$, and this restriction is still admissible by Lemma 3.5. The connected algebraic monodromy group associated with $Y^{\mathrm{sm}}$ is defined to be the connected algebraic monodromy group of the restriction of the VMHS defined in the last paragraph to $Y^{\prime s m}$, i.e. the neutral component of the Zariski closure of the image of $\pi_{1}\left(Y^{\prime \mathrm{sm}}, y^{\prime}\right) \rightarrow \pi_{1}\left(S^{\prime}, y^{\prime}\right) \rightarrow P$.

Let us briefly prove that the connected algebraic monodromy group associated with $Y^{\mathrm{sm}}$ is well-defined. Suppose that we have another covering $S^{\prime \prime} \stackrel{p^{\prime}}{\rightarrow} S^{\prime}$ with $S^{\prime \prime}$ smooth. Let $Y^{\prime \prime}$ be an 
irreducible component of $p^{-1}\left(Y^{\prime}\right)$. Let $Y_{0}^{\prime \prime s m}:=Y^{\prime \prime s m} \cap p^{-1}\left(Y^{\prime s m}\right)$, then by the commutative diagram

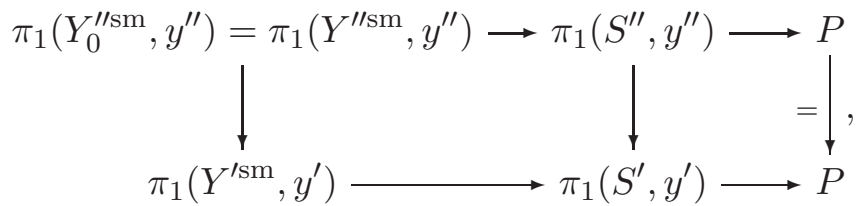

where the equality in the top-left cornor is given by $[37,2.10 .1]$ and the morphism on the left is of finite index by $[37,2.10 .2]$, the neutral components of the Zariski closures of the images of $\pi_{1}\left(Y^{\prime \prime s m}, y^{\prime \prime}\right)$ and $\pi_{1}\left(Y^{\prime s m}, y^{\prime}\right)$ in $P$ coincide.

Theorem 8.1. Let $S$ be a connected mixed Shimura variety associated with the connected mixed Shimura datum $\left(P, \mathcal{X}^{+}\right)$and let unif: $\mathcal{X}^{+} \rightarrow S=\Gamma \backslash \mathcal{X}^{+}$be the uniformization. Let $Y$ be a closed irreducible subvariety of $S$ and

- let $\tilde{Y}$ be an irreducible component of unif $^{-1}(Y)$;

- take $\widetilde{y}_{0} \in \tilde{Y}$;

- let $N$ be the connected algebraic monodromy group associated with $Y^{\mathrm{sm}}$.

Then

(1) The set $\widetilde{F}:=N(\mathbb{R})^{+} U_{N}(\mathbb{C}) \widetilde{y}_{0}$, where $U_{N}:=U \cap N$, is a weakly special subset of $\mathcal{X}^{+}$(or equivalently, $F:=\operatorname{unif}(\widetilde{F})$ is a weakly special subvariety of $S)$. Moreover $N$ is the largest subgroup of $Q$ s.t. $N(\mathbb{R})^{+} U_{N}(\mathbb{C})$ stabilizes $\widetilde{F}$, where $\left(Q, \mathcal{Y}^{+}\right)$is the smallest connected sub-mixed Shimura datum with $\widetilde{F} \subset \mathcal{Y}^{+}$;

(2) The Zariski closure of $\widetilde{Y}$ in $\mathcal{X}^{+}$(which means the complex analytic irreducible component of the intersection of the Zariski closure of $\widetilde{Y}$ in $\mathcal{X}^{\vee}$ and $\mathcal{X}^{+}$which contains $\left.\tilde{Y}\right)$ is $\widetilde{F}$;

(3) The smallest weakly special subset containing $\widetilde{Y}$ is $\widetilde{F}$ and $F$ is the smallest weakly special subvariety of $S$ containing $Y$.

Proof. (1) Let $S_{Y}$ be the smallest special subvariety containing $Y$. Such an $S_{Y}$ exists since the irreducible components of intersections of special subvarieties are special (which can easily be shown by means of generic Mumford-Tate group). By definition of special subvarieties, there exists a connected mixed Shimura subdatum $\left(Q, \mathcal{Y}^{+}\right)$s.t. $S_{Y}$ is the image of $\Gamma_{Q} \backslash \mathcal{Y}^{+}$in $S$ where $\Gamma_{Q}:=\Gamma \cap Q(\mathbb{Q})$. We may furthermore assume $\left(Q, \mathcal{Y}^{+}\right)$to have generic Mumford-Tate group by Proposition 2.6.

Let $N$ be the connected algebraic monodromy group associated with $Y^{\mathrm{sm}}$, then $N \triangleleft Q$ (and also $N \triangleleft Q^{\text {der }}$ ) by the discussion at the beginning of this section (which claims that the variation we use to define $N$ is admissible), Remark 3.8 (which claims that the generic Mumford-Tate group of this variation is $Q$ ) and Theorem 3.6.

Then $\widetilde{F}$ is a weakly special subset of $\mathcal{Y}^{+}$since it is the inverse image of the point $\varphi\left(\widetilde{y}_{0}\right)$ under the Shimura morphism $\left(Q, \mathcal{Y}^{+}\right) \stackrel{\varphi}{\rightarrow}\left(Q, \mathcal{Y}^{+}\right) / N$. Then $\widetilde{F}$ is also a weakly special subset of $\mathcal{X}^{+}$by definition. By the choice of $\left(Q, \mathcal{Y}^{+}\right), \widetilde{F}$ is Hodge generic in $\mathcal{Y}^{+}$, and

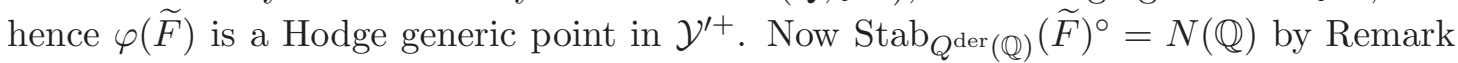
3.8 .

(2) We prove that $\widetilde{F}$ is the Zariski closure of $\widetilde{Y}$ in $\mathcal{X}^{+}$. We first show that it suffices to prove $\tilde{Y} \subset \widetilde{F}$. Let $\overline{\widetilde{Y}}$ be the Zariski closure of $\tilde{Y}$ in $\mathcal{X}^{+}$, then $\overline{\widetilde{Y}} \subset \widetilde{F}$ since $\widetilde{Y} \subset \widetilde{F}$ and 
$\widetilde{F}$ is algebraic (Lemma 6.2). On the other hand, $\Gamma_{Y^{\mathrm{sm}}}:=\operatorname{Im}\left(\pi_{1}\left(Y^{\mathrm{sm}}\right) \rightarrow \pi_{1}(S) \rightarrow P\right.$ ) stabilizes $\widetilde{Y}$, so $\Gamma_{Y^{\text {sm }}} \widetilde{y}_{0} \subset \widetilde{Y}$. The group $\Gamma_{Y^{\text {sm }}}$ is Zariski dense in $N$, and hence Zariski dense in $N_{\mathbb{C}}$. As $\widetilde{F}=N(\mathbb{C}) \widetilde{y}_{0} \cap \mathcal{X}^{+}, \Gamma_{Y^{\text {sm }}} \widetilde{y}_{0}$ is Zariksi dense in $\widetilde{F}$. Hence we have $\widetilde{F} \subset \overline{\widetilde{Y}}$. As a result, $\widetilde{F}=\overline{\widetilde{Y}}$.

Next we prove that $\widetilde{Y} \subset \widetilde{F}$ (or equivalently, $Y \subset F$ ).

The fact that $\widetilde{Y} \subset \widetilde{F}$ has nothing to do with the level structure. Hence we may assume $\Gamma=\Gamma_{W} \rtimes \Gamma_{G}$ with $\Gamma_{W} \subset W(\mathbb{Z}), \Gamma_{U}:=\Gamma_{W} \cap U \subset U(\mathbb{Z}), \Gamma_{V}:=\Gamma_{W} / \Gamma_{U} \subset V(\mathbb{Z})$ and $\Gamma_{G} \subset G(\mathbb{Z})$ small enough s.t. they are all neat and s.t. $\Gamma \subset P^{\operatorname{der}}(\mathbb{Q})(\operatorname{Remark} 2.2(2))$. We write $\Gamma_{P / U}:=\Gamma / \Gamma_{U}$.

We may replace $\left(P, \mathcal{X}^{+}\right)$by $\left(Q, \mathcal{Y}^{+}\right)$and $S$ by $S_{Y}$ (same notation as in (1)) since $\tilde{Y}$, $\widetilde{F} \subset \mathcal{Y}^{+}$and $Y, F \subset S_{Y}$. In other words, we may assume that $Y$ is Hodge generic in $S$ and $\left(P, \mathcal{X}^{+}\right)$is irreducible.

Consider the following diagram:

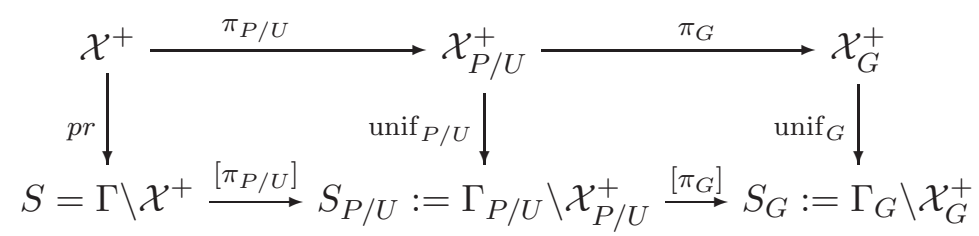

Denote by $\pi$ and $[\pi]$ the composites of the maps in the two lines respectively. Denote by $\widetilde{Y}_{G}:=\pi(\widetilde{Y}), Y_{G}:=[\pi](Y)$ and $\widetilde{Y}_{P / U}:=\pi_{P / U}(\widetilde{Y}), Y_{P / U}:=\left[\pi_{P / U}\right](Y) ; \widetilde{F}_{G}:=\pi(\widetilde{F})$, $F_{G}:=[\pi](F)$ and $\widetilde{F}_{P / U}:=\pi_{P / U}(\widetilde{F}), F_{P / U}:=\left[\pi_{P / U}\right](F)$. Denote by $\widetilde{y}_{0, P / U}:=\pi_{P / U}\left(\widetilde{y}_{0}\right)$ and $\widetilde{y}_{0, G}:=\pi\left(\widetilde{y}_{0}\right)$.

Now to make the proof more clear, we divide it into several steps.

Step I. Prove that $\widetilde{Y}_{G} \subset \widetilde{F}_{G}$.

We begin the proof with the following lemma:

Lemma 8.2. In the context above, the connected algebraic monodromy group associated with $\overline{Y_{G}}$ sm (resp. $\overline{Y_{P / U}}$ sm $)$ is $G_{N}\left(\right.$ resp. $N / U_{N}$ where $\left.U_{N}:=U \cap N\right)$.

Proof. We only prove the statement for ${\overline{Y_{G}}}^{\text {sm }}$. The proof for ${\overline{Y_{P / U}}}^{\text {sm }}$ is similar. Take $Y_{0}^{\mathrm{sm}}:=Y^{\mathrm{sm}} \cap \pi^{-1}\left(Y_{G}^{\mathrm{sm}}\right)$, then we have the commutative diagram below:

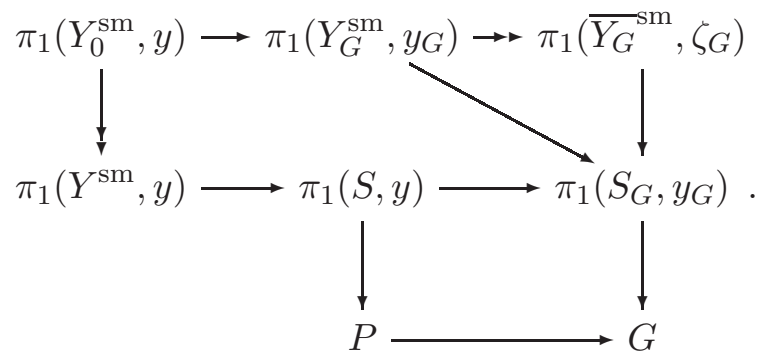

Here, the morphism on the left and the right morphism on the top are surjective since $\operatorname{codim}_{Y \text { sm }}\left(Y^{\mathrm{sm}}-Y_{0}^{\mathrm{sm}}\right) \geqslant 1$ and $\operatorname{codim}_{\overline{Y_{G}}} \mathrm{sm}\left({\overline{Y_{G}}}^{\mathrm{sm}}-Y_{G}^{\mathrm{sm}}\right) \geqslant 1([37,2.10 .1])$. Now [37, 2.10.2] shows that the image of $\pi_{1}\left(Y_{0}^{\mathrm{sm}}, y\right)$ is of finite index in $\pi_{1}\left(Y_{G}^{\mathrm{sm}}, y_{G}\right)$, so the neutral 
components of the Zariski closures of $\pi_{1}\left(Y^{\mathrm{sm}}, y\right)$ and $\pi_{1}\left({\overline{Y_{G}}}^{\mathrm{sm}}, y_{G}\right)$ in $G$ coincide. Hence we are done.

Let $\widetilde{Z}$ be the closure (w.r.t. archimedean topology) of $\widetilde{Y}_{G}$ in $\mathcal{X}_{G}^{+}$, then $\widetilde{Z}$ is a complex analytic irreducible component of $\operatorname{unif}_{G}^{-1}\left(\overline{Y_{G}}\right)$. For the pure connected Shimura datum $\left(G^{\text {ad }}, \mathcal{X}_{G}^{+}\right)$, we have a decomposition $([40,3.6])$

$$
\left(G^{\mathrm{ad}}, \mathcal{X}_{G}^{+}\right)=\left(G_{N}^{\mathrm{ad}}, \mathcal{X}_{G, 1}^{+}\right) \times\left(G_{2}, \mathcal{X}_{G, 2}^{+}\right) .
$$

By $[40,3.6,3.7]$ and Lemma 8.2, $\widetilde{Z} \subset \mathcal{X}_{G, 1}^{+} \times\left\{\widetilde{y_{G, 2}}\right\}$, i.e. $\widetilde{Z} \subset G_{N}(\mathbb{R})^{+} \widetilde{x}_{G}$ for some $\widetilde{x}_{G} \in \mathcal{X}_{G}^{+}$. But $\widetilde{y}_{0, G} \in \widetilde{Y}_{G} \subset \widetilde{Z}$, so $\widetilde{F}_{G}=G_{N}(\mathbb{R})^{+} \widetilde{y}_{0, G} \subset G_{N}(\mathbb{R})^{+} \widetilde{x}_{G}$. This implies that $\widetilde{F}_{G}=G_{N}(\mathbb{R})^{+} \widetilde{x}_{G}$. As a result, $\widetilde{Y}_{G} \subset \widetilde{Z} \subset \widetilde{F}_{G}$.

Step II. Consider the Shimura morphism

$$
\left(P, \mathcal{X}^{+}\right) \stackrel{\rho}{\rightarrow}\left(P^{\prime}, \mathcal{X}^{+\prime}\right):=\left(P, \mathcal{X}^{+}\right) / N .
$$

Then $\widetilde{F}=\rho^{-1}(\rho(\widetilde{F}))$ by definition of $\rho$. So in order to prove $\widetilde{Y} \subset \widetilde{F}$, it is enough to show that $\rho(\widetilde{Y}) \subset \rho(\widetilde{F})$. Hence we may replace $\left(P, \mathcal{X}^{+}\right)$by $\left(P^{\prime}, \mathcal{X}^{+\prime}\right)$. In other words, we may assume $N=\mathbf{1}$.

In this case $\widetilde{F}$ is just a point $\widetilde{x} \in \mathcal{X}^{+}$. Call $\widetilde{x}_{P / U}:=\pi_{P / U}(\widetilde{x}), \widetilde{x}_{G}:=\pi(\widetilde{x})$ and $x:=\operatorname{unif}(\widetilde{x}), x_{P / U}:=\operatorname{unif}_{P / U}\left(\widetilde{x}_{P / U}\right), x_{G}:=\operatorname{unif}_{G}\left(\widetilde{x}_{G}\right)$. Then since $Y_{G} \subset F_{G}$, we have $Y \subset E$ where $E$ is the fibre of $S \stackrel{[\pi]}{\longrightarrow} S_{G}$ over $x_{G}$. Denote by $A$ the fibre of $S_{P / U} \stackrel{[\pi]_{G}}{\longrightarrow} S_{G}$ over $x_{G}$ and $T$ the fibre of $S \stackrel{\left[\pi_{P / U}\right]}{\longrightarrow} S_{P / U}$ over $x_{P / U}$, then by $[53,3.13,3.14] A$ is an abelian variety and $T$ is an algebraic torus.

Step III. Prove that $\widetilde{Y}_{P / U} \subset \widetilde{F}_{P / U}$, i.e. $\widetilde{Y}_{P / U}=\left\{\widetilde{x}_{P / U}\right\}$.

$\overline{\mathrm{By}} \underline{\text { Step } I}, Y_{P / U} \subset A$. We have the following morphisms

$$
\pi_{1}\left(Y_{P / U}^{\mathrm{sm}}\right) \rightarrow \pi_{1}(A) \rightarrow \pi_{1}\left(S_{P / U}\right)=\Gamma_{P / U} \rightarrow P / U=V \rtimes G .
$$

The neutral component of the Zariski closure of $\pi_{1}\left(Y_{P / U}^{\mathrm{sm}}\right)\left(\right.$ resp. $\left.\pi_{1}(A)\right)$ in $P / U=V \rtimes G$ is $\mathbf{1}$ (resp. $V$ ), so the image of

$$
\pi_{1}\left(Y_{P / U}^{\mathrm{sm}}\right) \rightarrow \pi_{1}(A)
$$

is a finite group.

Now $Y_{P / U}$ is irreducible since $Y$ is irreducible. So by Proposition 7.4, $Y_{P / U} \subset A$ is a point. Equivalently, $\widetilde{Y}_{P / U}$ is a point. So $\widetilde{Y}_{P / U} \subset \widetilde{F}_{P / U}$ since $\widetilde{Y}_{P / U} \cap \widetilde{F}_{P / U} \neq \emptyset$ (both of them contain $\left.\widetilde{y}_{0, P / U}\right)$.

Step $I V$. Prove that $\widetilde{Y} \subset \widetilde{F}$, i.e. $\widetilde{Y}=\{\widetilde{x}\}$.

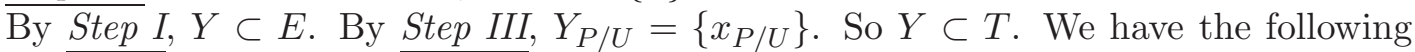
morphisms

$$
\pi_{1}\left(Y^{\mathrm{sm}}\right) \rightarrow \pi_{1}(T) \rightarrow \pi_{1}(S)=\Gamma \rightarrow P=W \rtimes G .
$$

The neutral component of the Zariski closure of $\pi_{1}\left(Y^{\mathrm{sm}}\right)$ (resp. $\left.\pi_{1}(T)\right)$ in $P=W \rtimes G$ is 1 (resp. $U$ ), so the image of

$$
\pi_{1}\left(Y^{\mathrm{sm}}\right) \rightarrow \pi_{1}(T)
$$

is a finite group. 
Now since $Y$ is irreducible, by Proposition 7.4, $Y \subset T$ is a point. Equivalently, $\widetilde{Y}$ is a point. So $\widetilde{Y} \subset \widetilde{F}$ since $\widetilde{Y} \cap \widetilde{F} \neq \emptyset$ (both of them contain $\widetilde{y}_{0}$ ).

(3) Since every weakly special subset of $\mathcal{X}^{+}$is algebraic by Lemma $6.2, \widetilde{F}$ is also the smallest weakly special subset which contains $\tilde{Y}$. Therefore $F$ is the smallest weakly special subvariety of $S$ which contains $Y$.

Corollary 8.3. Let $S$ be a connected mixed Shimura variety associated with the connected mixed Shimura datum $\left(P, \mathcal{X}^{+}\right)$and let unif: $\mathcal{X}^{+} \rightarrow S=\Gamma \backslash \mathcal{X}^{+}$be the uniformization map. Let $Y$ be a closed irreducible subvariety of $S$, then $Y$ is weakly special if and only if one (equivalently any) irreducible component of unif ${ }^{-1}(Y)$ is algebraic.

If $Y$ is weakly special, then $Y=\operatorname{unif}\left(N(\mathbb{R})^{+} U_{N}(\mathbb{C}) \widetilde{y}\right)$ where $N$ is the connected algebraic monodromy group associated with $Y^{\mathrm{sm}}, U_{N}:=U \cap N$ and $\widetilde{y}$ is any point of $\operatorname{unif}^{-1}(Y)$.

Proof. The "only if" part is immediate by Lemma 6.2. Now we prove the "if" part.

We first of all quickly show that if one irreducible component of $\operatorname{unif}^{-1}(Y)$ is algebraic, so are the others. The proof is the same as [62, first paragraph of the proof of Theorem 4.1]. Suppose that $\widetilde{Y}$ is an irreducible component of unif $^{-1}(Y)$ which is algebraic, i.e. $\widetilde{Y}$ is an irreducible component of $\mathcal{X}^{+} \cap Z$ for some algebraic subvariety $Z$ of $\mathcal{X}^{\vee}$. Then for any $\gamma \in \Gamma \subset P(\mathbb{R}) U(\mathbb{C})$,

$$
\gamma \widetilde{Y}=\gamma\left(\mathcal{X}^{+} \cap Z\right) \subset \mathcal{X}^{+} \cap \gamma Z=\gamma \gamma^{-1}\left(\mathcal{X}^{+} \cap \gamma Z\right) \subset \gamma \widetilde{Y} \text {. }
$$

Hence it follows that $\gamma \widetilde{Y}=\mathcal{X}^{+} \cap \gamma Z$ is algebraic.

Next under the notation of Theorem 8.1, $\widetilde{Y}=\overline{\widetilde{Y}}=\widetilde{F}$ since $\widetilde{Y}$ is algebraic. Hence $\widetilde{Y}$ is weakly special, and so is $Y$.

Finally if $Y$ is weakly special, then for any $\widetilde{y} \in$ unif $^{-1}(Y)$ and $\widetilde{Y}$ the irreducible component of unif ${ }^{-1}(Y)$ which contains $\widetilde{y}, \widetilde{Y}=\widetilde{F}=N(\mathbb{R})^{+} U_{N}(\mathbb{C}) \widetilde{y}$ by Theorem 8.1, and hence $Y=$ $\operatorname{unif}\left(N(\mathbb{R})^{+} U_{N}(\mathbb{C}) \widetilde{y}\right)$.

\section{Ax-Lindemann-Weierstrass Part 1: Outline of the proof}

In the following three sections, we are going to prove Theorem 1.2. The organization of the proof is as follows: the outline of the proof is given in this section. After some preparation, the key proposition (Proposition 9.6) leading to the theorem will be stated and exploited (together with Theorem 7.6) to finish the proof in Theorem 9.8. We prove this key proposition in the next section using Pila-Wilkie's counting theorem and Theorem 7.6 will be proved in $\S 11$, where a simple proof of Ax-Lindemann-Weierstraß for complex abelian varieties can be found.

Now let us fix some notation which will be used through the whole proof:

Notation 9.1. Consider the following diagram:

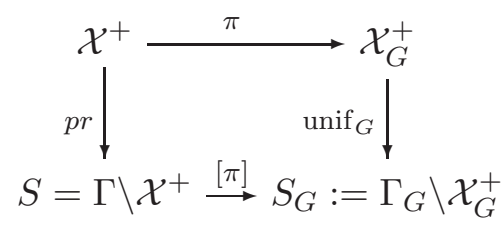

Denote by $\widetilde{Y}_{G}:=\pi(\widetilde{Y}), Y_{G}:=[\pi](Y)$ and $\widetilde{Z}_{G}:=\pi(\widetilde{Z})$. 
Now we begin the proof of Theorem 1.2. Let us first of all do some reduction:

- Since every point of $\mathcal{X}^{+}$is weakly special, we may assume $\operatorname{dim}(\widetilde{Z})>0$.

- Let $\left(Q, \mathcal{Y}^{+}\right)$be the smallest mixed Shimura subdatum of $\left(P, \mathcal{X}^{+}\right)$s.t $\widetilde{Z} \subset \mathcal{Y}^{+}$and let $S_{Q}$ be the corresponding special subvariety of $S$. Then $Q=\operatorname{MT}\left(\mathcal{Y}^{+}\right)$by Proposition 2.6(1). If we replace $\left(P, \mathcal{X}^{+}\right)$by $\left(Q, \mathcal{Y}^{+}\right), S$ by $S_{Q}$, unif: $\mathcal{X}^{+} \rightarrow S$ by unif ${ }_{Q}: \mathcal{Y}^{+} \rightarrow S_{Q}$ and $Y$ by an irreducible component $Y_{0}$ of $Y \cap S_{Q}$, then $\widetilde{Z}$ is again a maximal irreducible algebraic subset of $\operatorname{unif}_{Q}^{-1}\left(Y_{0}\right)$. By definition, $\widetilde{Z}$ is weakly special in $\mathcal{X}^{+}$iff it is weakly special in $\mathcal{Y}^{+}$. So we may assume $P=\operatorname{MT}\left(\mathcal{X}^{+}\right)$and that $\widetilde{Z}$ is Hodge generic.

- Furthermore, let $Y_{0}$ by the minimal irreducible subvariety of $S$ such that $\widetilde{Z} \subset$ unif $^{-1}\left(Y_{0}\right)$, then $\widetilde{Z}$ is still maximal irreducible algebraic in $\operatorname{unif}^{-1}\left(Y_{0}\right)$. Hence we may assume that $Y=Y_{0}$. In fact it is not hard to see that after this reduction, $Y=\overline{\operatorname{unif}(\widetilde{Z})}$ and $\widetilde{Z}$ is weakly special iff $Y$ is weakly special.

- By the previous reduction, there is a unique complex analytic irreducible component of unif $^{-1}(Y)$ which contains $\widetilde{Z}$. Denote it by $\widetilde{Y}$. Denote by $\widetilde{Y}_{G}:=\pi(\widetilde{Y}), Y_{G}:=[\pi](Y)$ and $\widetilde{Z}_{G}:=\pi(\widetilde{Z})$. Remark that by Lemma $6.3, \widetilde{Z}_{G}$ is an algebraic subset of $\mathcal{X}_{G}^{+}$.

- Replacing $\Gamma$ by a subgroup of finite index does not matter for this problem, so we may assume that $\Gamma$ is neat and $\Gamma \subset P^{\text {der }}(\mathbb{Q})($ Remark $2.2(2))$.

Let $\widetilde{F}$ be the smallest weakly special subset containing $\widetilde{Y}$. By Theorem 8.1, $\widetilde{F}=N(\mathbb{R})^{+} U_{N}(\mathbb{C}) \widetilde{z}$ some $\widetilde{z} \in \widetilde{Z} \subset \widetilde{Y}$, where $N$ is the connected algebraic monodromy group associated with $Y^{\text {sm }}$ and $U_{N}:=U \cap N$. The set $\widetilde{F}$ is Hodge generic in $\left(P, \mathcal{X}^{+}\right)$since $\widetilde{Z}$ is, so $N \triangleleft P$ and $N \triangleleft P^{\text {der }}$ by Theorem 3.6.

Define

$$
\Gamma_{\widetilde{Z}}:=\{\gamma \in \Gamma \mid \gamma \cdot \widetilde{Z}=\widetilde{Z}\} \quad\left(\operatorname{resp} . \Gamma_{G}, \widetilde{Z}_{G}:=\left\{\gamma_{G} \in \Gamma_{G} \mid \gamma_{G} \cdot \overline{\widetilde{Z}_{G}}=\overline{\widetilde{Z}_{G}}\right\}\right)
$$

and

$$
H_{\widetilde{Z}}:=\left({\overline{\Gamma_{\widetilde{Z}}}}^{\mathrm{Zar}}\right)^{\circ} \quad\left(\operatorname{resp} . H_{\widetilde{Z}_{G}}:=\left({\overline{\Gamma_{G,} \overline{\widetilde{Z}}_{G}}}^{\mathrm{Zar}}\right)^{\circ}\right) .
$$

Define $U_{H_{\widetilde{Z}}}:=U \cap H_{\widetilde{Z}}$ and $W_{H_{\widetilde{Z}}}:=W \cap H_{\widetilde{Z}}$. Both of them are normal in $H_{\widetilde{Z}}$. Then $H_{\widetilde{Z}}$ (resp. $\left.H_{\widetilde{Z}_{G}}\right)$ is the largest connected subgroup of $P^{\text {der }}$ (resp. $G^{\text {der }}$ ) such that $H_{\widetilde{Z}}(\mathbb{R})^{+} U_{H_{\widetilde{Z}}}(\mathbb{C})$ (resp. $H \widetilde{Z}_{G}(\mathbb{R})^{+}$) stabilizes $\widetilde{Z}$ (resp. $\widetilde{\widetilde{Z}}_{G}$ ).

Define $V_{H_{\widetilde{Z}}}:=W_{H_{\widetilde{Z}}} / U_{H_{\widetilde{Z}}}$ and $G_{H_{\widetilde{Z}}}:=H_{\widetilde{Z}} / W_{H_{\widetilde{Z}}} \hookrightarrow P / W=G$.

The following two lemmas were proved for the pure case in [49] and [35].

Lemma 9.2. The set $\widetilde{Y}$ is stable under $H_{\widetilde{Z}}(\mathbb{R})^{+} U_{H_{\widetilde{Z}}}(\mathbb{C})$.

Proof. Every fiber of $\mathcal{X}^{+} \rightarrow \mathcal{X}_{P / U}^{+}$can be canonically identified with $U(\mathbb{C})$. So it is enough to prove that $\widetilde{Y}$ is stable under $H_{\widetilde{Z}}(\mathbb{R})^{+}$: If $U_{H_{\widetilde{Z}}}(\mathbb{R}) \widetilde{y} \subset \widetilde{Y}$ for $\widetilde{y} \in \widetilde{Y}$, then $U_{H_{\tilde{Z}}}(\mathbb{C}) \widetilde{y} \subset \widetilde{Y}$ because $\widetilde{Y}$ is complex analytic and $U_{H_{\tilde{Z}}}(\mathbb{C}) \widetilde{y}$ is the smallest complex analytic subset of $\mathcal{X}^{+}$containing $U_{H_{\widetilde{Z}}}(\mathbb{R}) \widetilde{y}$.

If not, then since $H_{\widetilde{Z}}(\mathbb{Q})$ is dense (w.r.t. the archimedean topology) in $H_{\widetilde{Z}}(\mathbb{R})^{+}$, there exists $h \in H_{\widetilde{Z}}(\mathbb{Q})$ such that $h \widetilde{Y} \neq \widetilde{Y}$. The set $\widetilde{Z}$ is contained in $\widetilde{Y} \cap h \widetilde{Y}$ by definition of $H_{\widetilde{Z}}$, and hence contained in a complex analytic irreducible component $\widetilde{Y}^{\prime}$ of it. 
Consider the Hecke operator $T_{h}$. Then $T_{h}(Y)=\operatorname{unif}\left(h \cdot \operatorname{unif}^{-1}(Y)\right)$. Hence

$$
Y \cap T_{h}(Y)=\operatorname{unif}\left(\operatorname{unif}^{-1}(Y) \cap\left(h \cdot \operatorname{unif}^{-1}(Y)\right)\right) .
$$

On the other hand, $T_{h}(Y)$ is equidimensional of the same dimension as $Y$ by definition, hence by reason of dimension, $h \widetilde{Y}$ is an irreducible component of unif ${ }^{-1}\left(T_{h}(Y)\right)=\Gamma h \Gamma \tilde{Y}$. So unif $(h \tilde{Y})$ is an irreducible component of $T_{h}(Y)$.

Since $\tilde{Y}^{\prime}$ is a complex analytic irreducible component of $\tilde{Y} \cap h \tilde{Y}$, it is also a complex analytic irreducible component of unif ${ }^{-1}(Y) \cap(h \widetilde{Y})=\Gamma \widetilde{Y} \cap h \widetilde{Y}$. So $Y^{\prime}:=\operatorname{unif}\left(\widetilde{Y}^{\prime}\right)$ is a complex analytic irreducible component of $Y \cap \operatorname{unif}(h \tilde{Y})$. So $Y^{\prime}$ is a complex analytic irreducible component of $Y \cap T_{h}(Y)$, and hence is algebraic since $Y \cap T_{h}(Y)$ is.

Since $h \widetilde{Y} \neq \widetilde{Y}$ and $Y$ is irreducible, $\operatorname{dim}\left(Y^{\prime}\right)<\operatorname{dim}(Y)$. But $\widetilde{Z} \subset \widetilde{Y} \cap h \widetilde{Y} \subset \operatorname{unif}^{-1}\left(Y^{\prime}\right)$. This contradicts the minimality of $Y$.

Lemma 9.3. $H_{\widetilde{Z}} \triangleleft N$.

Proof. We have $\widetilde{Z} \subset \widetilde{F}=N(\mathbb{R})^{+} U_{N}(\mathbb{C}) \widetilde{z}$ for some $\widetilde{z} \in \widetilde{Z}$, so the image of $\widetilde{Z}$ under the morphism

$$
\left(P, \mathcal{X}^{+}\right) \rightarrow\left(P, \mathcal{X}^{+}\right) / N
$$

is a point. But $H_{\widetilde{Z}} /\left(H_{\widetilde{Z}} \cap N\right)$ stabilizes this point which is Hodge generic (since $\widetilde{F}$ is Hodge generic in $\mathcal{X}^{+}$), and therefore is trivial by Remark 3.8. So $H_{\widetilde{Z}}<N$.

Let $H^{\prime}$ be the algebraic group generated by $\gamma^{-1} H_{\widetilde{Z}} \gamma$ for all $\gamma \in \Gamma_{Y^{\text {sm }}}$, where $\Gamma_{Y^{\text {sm }}}$ is the monodromy group of $Y^{\mathrm{sm}}$. Since $H^{\prime}$ is invariant under conjugation by $\Gamma_{Y^{\mathrm{sm}}}$, it is invariant under $\overline{\Gamma_{Y \text { sm }}}$ Zar , therefore invariant under conjugation by $N$.

By Lemma $9.2, \widetilde{Y}$ is invariant under $H_{\widetilde{Z}}(\mathbb{R})^{+} U_{H_{\widetilde{Z}}}(\mathbb{C})$. On the other hand, $\widetilde{Y}$ is also invariant under $\Gamma_{Y^{\text {sm }}}$ by definition. So $\tilde{Y}$ is invariant under the action of $H^{\prime}(\mathbb{R})^{+} U_{H^{\prime}}(\mathbb{C})$ where $U_{H^{\prime}}:=$ $U \cap H^{\prime}$. Since $H^{\prime}(\mathbb{R})^{+} U_{H^{\prime}}(\mathbb{C}) \widetilde{Z}$ is semi-algebraic, there exists an irreducible algebraic subset of $\mathcal{X}^{+}$, say $\widetilde{E}$, which contains $H^{\prime}(\mathbb{R})^{+} U_{H^{\prime}}(\mathbb{C}) \widetilde{Z}$ and is contained in $\widetilde{Y}$ by [50, Lemma 4.1]. Now $\widetilde{Z} \subset \widetilde{E} \subset \widetilde{Y}$, so $\widetilde{Z}=\widetilde{E}=H^{\prime}(\mathbb{R})^{+} U_{H^{\prime}}(\mathbb{C}) \widetilde{Z}$ by maximality of $\widetilde{Z}$, and therefore $H^{\prime}=H_{\widetilde{Z}}$ by definition of $H_{\widetilde{Z}}$. So $H_{\widetilde{Z}}$ is invariant under conjugation by $N$. Since $H_{\widetilde{Z}}<N, H_{\widetilde{Z}}$ is normal in $N$.

\section{Corollary 9.4 .}

$$
G_{H_{\widetilde{Z}}}, H \overline{\widetilde{Z}}_{G} \triangleleft G^{\text {der }} \text { and } G_{H_{\widetilde{Z}}} \triangleleft H \bar{Z}_{\widetilde{Z}_{G}} \text {. }
$$

Proof. We have $G_{H_{\widetilde{Z}}} \triangleleft G_{N} \triangleleft G^{\text {der }}$, and so $G_{H_{\widetilde{Z}}} \triangleleft G^{\text {der }}$ since all the three groups are reductive.

Working with $\left(\left(G, \mathcal{X}_{G}^{+}\right), \overline{Y_{G}}, \widetilde{Z}_{G}\right)$ instead of $\left(\left(P, \mathcal{X}^{+}\right), Y, \widetilde{Z}\right)$, we can prove (similar to Lemma 9.3) that $H_{\widetilde{Z}_{G}} \triangleleft G_{N}$. Hence $H_{\widetilde{Z}_{G}} \triangleleft G^{\text {der }}$ by the same reason for $G_{H_{\widetilde{Z}}}$.

By definition $G_{H_{\widetilde{Z}}}<H_{\widetilde{Z}_{G}}$. So $G_{H_{\widetilde{Z}}} \triangleleft H_{\widetilde{Z}_{G}}$ since $G_{H_{\widetilde{Z}}} \triangleleft G^{\text {der }}$.

So far the proof looks similar to the pure case. From now on it will be quite different. For the readers' convenience, we list here some differences between the proof of Ax-LindemannWeierstraß for mixed Shimura varieties and for the pure case:

- We shall prove that $\widetilde{Z}$ is an $H_{\widetilde{Z}}(\mathbb{R})^{+} U_{H_{\widetilde{Z}}}(\mathbb{C})$-orbit. To prove this, it suffices to prove $\operatorname{dim} H_{\widetilde{Z}}>0$ when $S$ is a pure Shimura variety. However this is far from enough for the 
mixed case, since this does not exclude the naive counterexample when $\operatorname{dim} \widetilde{Z}_{G}>0$ but $H_{\widetilde{Z}}$ is unipotent. To overcome it, we should at least prove $\operatorname{dim} G_{H_{\widetilde{Z}}}>0$. In fact we shall directly prove $G_{H_{\widetilde{Z}}}=H_{\widetilde{Z}_{G}}$ (Proposition 9.6). This equality is not obvious because, as appears in the proof of Lemma 9.5, there is no reason a priori why $\overline{\widetilde{Z}}_{G}$, which is obviously algebraic in unif ${ }^{-1}\left(Y_{G}\right)$, should be maximal for this property. If one could prove direcly this is the case, then Klingler-Ullmo-Yafaev [35, Theorem 1.3] would give directly the result.

- As mentioned in the Introduction, we shall make essential use of the "family" version of Pila-Wilkie's theorem (Remark 10.4);

- If $P=G$ is reductive, then $H_{\widetilde{Z}} \triangleleft N \triangleleft P$ implies directly $H_{\widetilde{Z}} \triangleleft P$. This is obviously false when $P$ is not reductive.

- For a general mixed Shimura variety $S$, the fiber of $S \stackrel{[\pi]}{\longrightarrow} S_{G}$ is not necessarily an algebraic group (Lemma 7.1), hence not a semi-abelian variety. We do not have AxLindemann-Weierstraß for the fiber for this case. Thus we should execute a proof of Ax-Lindemann-Weierstraß for the fiber. As the readers will see in $\S 11$, the proof of this case calls for much more careful study of $\widetilde{Z}$. First of all, when doing the estimate and using the family version of Pila-Wilkie for the fiber (Step I), we should introduce a seemingly strange subgroup which serves as $G_{N}$ in the section. The reason for this will be explained in Remark 11.1. Secondly, to prove that $W_{H_{\widetilde{Z}}}$ is normal in $W$ is not trivial, and the key to the solution (Step $I V$ ) is a well-known fact: any holomorphic morphism from a complex abelian variety to an algebraic torus over $\mathbb{C}$ is trivial.

Before proceeding, we prove the following lemma:

Lemma 9.5. (1) $\overline{\widetilde{Y}}_{G}$ is weakly special. Hence $\overline{\widetilde{Y}_{G}}=G_{N}(\mathbb{R})^{+} \widetilde{z}_{G}$ for any point $\widetilde{z}_{G} \in \widetilde{Z}_{G}$;

(2) $\overline{\operatorname{unif}_{G}\left(\widetilde{Z}_{G}\right)}=\overline{Y_{G}}$.

Proof. (1) Let $\widetilde{Z}^{\prime}$ be an irreducible algebraic subset of $\mathcal{X}_{G}^{+}$which contains $\widetilde{Z}_{G}$ and is contained in $\operatorname{unif}^{-1}\left(\overline{Y_{G}}\right)$, maximal for these properties. By [35, Theorem 1.3], $Z^{\prime}:=\operatorname{unif}_{G}\left(\widetilde{Z}^{\prime}\right)$ is weakly special, and therefore Zariski closed by definition. Now $\widetilde{Z} \subset \pi^{-1}\left(\widetilde{Z}^{\prime}\right) \cap \operatorname{unif}^{-1}(Y)$. However,

$$
\operatorname{unif}\left(\pi^{-1}\left(\widetilde{Z}^{\prime}\right) \cap \operatorname{unif}^{-1}(Y)\right)=\operatorname{unif}\left(\pi^{-1}\left(\widetilde{Z}^{\prime}\right)\right) \cap Y=[\pi]^{-1}\left(Z^{\prime}\right) \cap Y .
$$

Then we must have $Y \subset[\pi]^{-1}\left(Z^{\prime}\right)$ since $Y$ is the minimal irreducible closed subvariety of $S$ such that $\widetilde{Z} \subset$ unif $^{-1}(Y)$. Therefore $\overline{Y_{G}} \subset Z^{\prime}$. But $Z^{\prime} \subset \overline{Y_{G}}$ by definition of $Z^{\prime}$, so $Z^{\prime}=\overline{Y_{G}}$. This means that $\overline{Y_{G}}$ is weakly special.

(2) Let $Y^{\prime}:=\overline{\operatorname{unif}_{G}\left(\widetilde{Z}_{G}\right)}$, then $\overline{\widetilde{Z}_{G}} \subset \operatorname{unif}_{G}^{-1}\left(Y^{\prime}\right)$. So $\widetilde{Z} \subset \pi^{-1}\left(\operatorname{unif}_{G}^{-1}\left(Y^{\prime}\right)\right)=\operatorname{unif}^{-1}\left([\pi]^{-1}\left(Y^{\prime}\right)\right)$, and so

$$
\widetilde{Z} \subset \operatorname{unif}^{-1}\left([\pi]^{-1}\left(Y^{\prime}\right)\right) \cap \operatorname{unif}^{-1}(Y)=\operatorname{unif}^{-1}\left([\pi]^{-1}\left(Y^{\prime}\right) \cap Y\right) .
$$

Hence there exists an irreducible component $Y^{\prime \prime}$ of $[\pi]^{-1}\left(Y^{\prime}\right) \cap Y$ such that $\widetilde{Z} \subset$ unif $^{-1}\left(Y^{\prime \prime}\right)$. But

$$
[\pi]\left(Y^{\prime \prime}\right) \subset[\pi]\left([\pi]^{-1}\left(Y^{\prime}\right) \cap Y\right)=Y^{\prime} \cap Y_{G},
$$


so $\operatorname{dim}\left([\pi]\left(Y^{\prime \prime}\right)\right) \leqslant \operatorname{dim}\left(Y^{\prime} \cap Y_{G}\right)$. If $Y^{\prime} \neq \overline{Y_{G}}$, then $\operatorname{dim}\left(Y^{\prime} \cap Y_{G}\right)<\operatorname{dim}\left(Y_{G}\right)$ and therefore $\operatorname{dim}\left(Y^{\prime \prime}\right)<\operatorname{dim}(Y)$, which contradicts the minimality of $Y$. So $Y^{\prime}=\overline{Y_{G}}$.

Proposition 9.6 (key proposition). The set $\overline{\widetilde{Z}}_{G}$ is weakly special and $G_{H_{\widetilde{Z}}}=H_{\widetilde{Z}_{G}}$. In other words,

$$
\overline{\widetilde{Z}_{G}}=G_{H_{\widetilde{Z}}}(\mathbb{R})^{+} \widetilde{z}_{G}
$$

for any point $\widetilde{z}_{G} \in \widetilde{Z}_{G}$.

Now let us show how this proposition together with Theorem 7.6 implies Theorem 1.2. Before proceeding to the final argument, we shall prove the following group theoretical lemma:

Lemma 9.7. Fixing a Levi decomposition $H_{\widetilde{Z}}=W_{H_{\widetilde{Z}}} G_{H_{\tilde{Z}}}$, there exists a compatible Levi decomposition $P=W \rtimes G$.

Proof. Suppose that the fixed Levi decomposition of $H_{\widetilde{Z}}$ is given by $s_{1}: G_{H_{\widetilde{Z}}} \rightarrow H_{\widetilde{Z}}$. Define $P_{*}:=\pi^{-1}\left(G_{H_{\widetilde{Z}}}\right)$, then $H_{\widetilde{Z}}<P_{*}$. Now choose any Levi decomposition $P=W \rtimes G$ defined by $s_{2}: G \rightarrow P$. Then $G_{H_{\tilde{Z}}}$, being a subgroup of $G$, is realized as a subgroup of $P$ via $s_{2}$. Hence $s_{2}$ induces a Levi-decomposition $P_{*}=W \rtimes^{s_{2}} G_{H_{\tilde{Z}}}$. We have thus a diagram

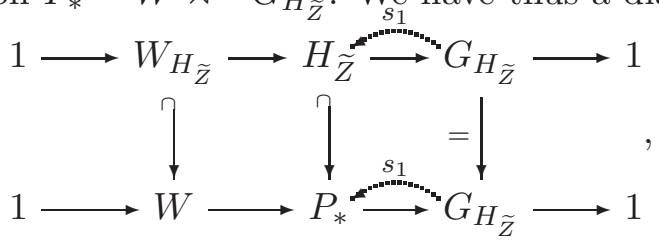

where the morphism $s_{1}$ in the second line is induced by the one in the first line. Now $s_{1}, s_{2}$ define two Levi decompositions of $P_{*}$. They differ by the conjugation by an element $w_{0}$ of $W(\mathbb{Q})$ by [55, Theorem 2.3]. So replacing $s_{2}$ by its conjugation by $w_{0}$ we can find a Levi decomposition of $P$ which is compatible with the fixed $H_{\widetilde{Z}}=W_{H_{\widetilde{Z}}} \rtimes G_{H_{\widetilde{Z}}}$.

Theorem 9.8.

(2) $H_{\widetilde{Z}} \triangleleft P$.

$$
\text { (1) } \widetilde{Z}=H_{\widetilde{Z}}(\mathbb{R})^{+} U_{H_{\widetilde{Z}}}(\mathbb{C}) \widetilde{z} \text { for any } \widetilde{z} \in \widetilde{Z} \text {; }
$$

Hence $\widetilde{Z}$ is weakly special by definition.

Proof. (1) Consider a fibre of $\widetilde{Z}$ over a Hodge-generic point $\widetilde{z}_{G} \in \widetilde{Z}_{G}$ such that $\left.\pi\right|_{\widetilde{Z}}$ is flat at $\widetilde{z}_{G}$ (such a point exists by $[1, \S 4$, Lemma 1.4] and generic flatness). Suppose that $\widetilde{W}$ is an irreducible algebraic component of $\widetilde{Z}_{\widetilde{z}_{G}}$ such that $\operatorname{dim}\left(\widetilde{Z}_{\widetilde{z}_{G}}\right)=\operatorname{dim}(\widetilde{W})$, then since $\left.\pi\right|_{\widetilde{Z}}$ is flat at $\widetilde{z}_{G}$,

$$
\operatorname{dim}(\widetilde{Z})=\operatorname{dim}\left(\widetilde{Z}_{G}\right)+\operatorname{dim}\left(\widetilde{Z}_{\widetilde{z}_{G}}\right)=\operatorname{dim}\left(\widetilde{Z}_{G}\right)+\operatorname{dim}(\widetilde{W}) .
$$

Consider the set $\widetilde{E}:=H_{\widetilde{Z}}(\mathbb{R})^{+} U_{H_{\widetilde{Z}}}(\mathbb{C}) \widetilde{W}$. It is semi-algebraic (since $\widetilde{W}$ is algebraic and the action of $P(\mathbb{R})^{+} U(\mathbb{C})$ on $\mathcal{X}^{+}$is algebraic). The fact $\widetilde{W} \subset \widetilde{Z}$ implies that $\widetilde{E} \subset \widetilde{Z}$. By [50, Lemma 4.1], there exists an irreducible algebraic subset of $\mathcal{X}^{+}$, say $\widetilde{E}_{\text {alg }}$, which contains $\widetilde{E}$ and is contained in $\widetilde{Z}$. Now we have by Proposition 9.6

$$
\pi(\widetilde{E})=G_{H_{\widetilde{Z}}}(\mathbb{R})^{+} \widetilde{z}_{G}=H_{\widetilde{Z}_{G}}(\mathbb{R})^{+} \widetilde{z}_{G}=\widetilde{Z}_{G}
$$


and that the $\mathbb{R}$-dimension of every fiber of $\left.\pi\right|_{\widetilde{E}}$ is at least $\operatorname{dim}_{\mathbb{R}}(\widetilde{W})$. So

$$
\operatorname{dim}\left(\widetilde{E}_{\mathrm{alg}}\right) \geqslant \operatorname{dim}(\pi(\widetilde{E}))+\operatorname{dim}(\widetilde{W})=\operatorname{dim}\left(\widetilde{Z}_{G}\right)+\operatorname{dim}(\widetilde{W})=\operatorname{dim}(\widetilde{Z}) .
$$

So $\widetilde{E}=\widetilde{Z}$ since $\widetilde{Z}$ is irreducible.

Next let $\widetilde{W}^{\prime}$ be an irreducible algebraic subset which contains $\widetilde{Z}_{\widetilde{z}_{G}}$ and is contained in unif $^{-1}(Y)_{\widetilde{z}_{G}}$, maximal for these properties. Then $\widetilde{W}^{\prime}$ is weakly special by Theorem 7.6. We have $\widetilde{W}^{\prime} \subset \widetilde{Y}$ since $\widetilde{Y}$ is an irreducible component of $\pi^{-1}(Y)$. Consider $\widetilde{E}^{\prime}:=$ $H_{\widetilde{Z}}(\mathbb{R})^{+} U_{H_{\widetilde{Z}}}(\mathbb{C}) \widetilde{W}^{\prime}$. Then $\widetilde{E}^{\prime} \subset \widetilde{Y}$ by Lemma 9.2. But $\widetilde{E}^{\prime}$ is semi-algebraic, so by [50, Lemma 4.1], there exists an irreducible algebraic subset of $\mathcal{X}^{+}$, say $\widetilde{E}_{\text {alg }}^{\prime}$ which contains $\widetilde{E}^{\prime}$ and is contained in $\widetilde{Y}$. So $\widetilde{Z}=\widetilde{E} \subset \widetilde{E}_{\text {alg }}^{\prime} \subset \widetilde{Y}$, and hence $\widetilde{Z}=\widetilde{E}_{\text {alg }}^{\prime}=\widetilde{E}^{\prime}$ by the maximality of $\widetilde{Z}$. So $\widetilde{Z}_{\widetilde{z}_{G}}=\widetilde{W}^{\prime}$ is weakly special.

Write $\widetilde{Z}_{\widetilde{z}_{G}}=W^{\prime}(\mathbb{R}) U^{\prime}(\mathbb{C}) \widetilde{z}$ with $W^{\prime}<W, U^{\prime}=W^{\prime} \cap U$ and $\widetilde{z} \in \widetilde{Z}_{\widetilde{z}_{G}}$. Then $W_{H_{\widetilde{Z}}}<$ $W^{\prime}$. The complex structure of $\pi^{-1}\left(\widetilde{z}_{G}\right)$ comes from $W(\mathbb{R}) U(\mathbb{C}) \simeq W(\mathbb{C}) / F_{\widetilde{z}_{G}}^{0} W(\mathbb{C})$, where $F_{\widetilde{z}_{G}}^{0} W(\mathbb{C})=\exp \left(F_{\widetilde{z}_{G}}^{0}\right.$ Lie $\left.W_{\mathbb{C}}\right)$. So the fact that $\widetilde{Z}_{\widetilde{z}_{G}}$ is a complex subspace of $\pi^{-1}\left(\widetilde{z}_{G}\right)$ implies that $W^{\prime} / U^{\prime}$ is a $\operatorname{MT}\left(\widetilde{z}_{G}\right)=G$-module. Hence $W^{\prime}$ is a $G$-group.

Define $P^{\prime}:=W^{\prime} H_{\widetilde{Z}}$, then $P^{\prime}$ is a subgroup of $P$ since $W^{\prime}>W_{H_{\widetilde{Z}}}$ and $G_{H_{\widetilde{Z}}} W^{\prime}=W^{\prime}$. Now we have

$$
\widetilde{Z}=H_{\widetilde{Z}}(\mathbb{R})^{+} U_{H_{\widetilde{Z}}}(\mathbb{C}) \widetilde{Z}_{\widetilde{z}_{G}}=H_{\widetilde{Z}}(\mathbb{R})^{+} U_{H_{\widetilde{Z}}}(\mathbb{C}) W^{\prime}(\mathbb{R}) U^{\prime}(\mathbb{C}) \widetilde{z}=P^{\prime}(\mathbb{R})^{+} U^{\prime}(\mathbb{C}) \widetilde{z}
$$

So $H_{\widetilde{Z}}=P^{\prime}$ because $H_{\widetilde{Z}}$ is the largest subgroup of $P^{\text {der }}$ such that $H_{\widetilde{Z}}(\mathbb{R})^{+} U_{H_{\widetilde{Z}}}(\mathbb{C})$ stabilizes $\widetilde{Z}$. So we have $\widetilde{Z}=H_{\widetilde{Z}}(\mathbb{R})^{+} U_{H_{\widetilde{Z}}}(\mathbb{C}) \widetilde{z}$.

(2) First of all, $U_{H_{\widetilde{Z}}} \triangleleft P$ by Proposition 2.6(2).

Next consider the complex structure of $\pi^{-1}\left(\widetilde{z}_{G}\right)$ which comes from $W(\mathbb{R}) U(\mathbb{C}) \simeq$ $W(\mathbb{C}) / F_{\widetilde{z}_{G}}^{0} W(\mathbb{C})$. So the fact that $\widetilde{Z}_{\widetilde{z}_{G}}$ is a complex subspace of $\pi^{-1}\left(\widetilde{z}_{G}\right)$ implies that $V_{H_{\tilde{Z}}}$ is a $\operatorname{MT}\left(\widetilde{z}_{G}\right)=G$-module. Hence $W_{H_{\widetilde{Z}}}$ is a $G$-group. Besides, $G_{H_{\tilde{Z}}} \triangleleft G$ by Proposition 9.6. In particular, $G_{H_{\widetilde{Z}}}$ is reductive.

Then let us prove $W_{H_{\widetilde{Z}}} \triangleleft P$. It suffices to prove $W_{H_{\widetilde{Z}}} \triangleleft W$. For any $\widetilde{z} \in \widetilde{Z}$, we have proved in (1) that $\widetilde{Z}_{\widetilde{z}_{G}}=W_{H_{\widetilde{Z}}}(\mathbb{R}) U_{H_{\widetilde{Z}}}(\mathbb{C}) \widetilde{z}$ is weakly special. Hence by Proposition 5.4, there is a connected mixed Shimura subdatum $\left(Q, \mathcal{Y}^{+}\right) \hookrightarrow\left(P, \mathcal{X}^{+}\right)$such that $\widetilde{z} \in \mathcal{Y}^{+}$ and $W_{H_{\tilde{Z}}} \triangleleft Q$. Define $W^{*}$ to be the $G$-subgroup (of $W$ ) generated by $W_{Q}:=\mathcal{R}_{u}(Q$ ), then $W_{H_{\widetilde{Z}}} \triangleleft W^{*}$ since $W_{H_{\widetilde{Z}}}$ is a $G$-group.

Fix a Levi decomposition $H_{\widetilde{Z}}=W_{H_{\widetilde{Z}}} \rtimes G_{H_{\widetilde{Z}}}$ and choose a compatible Levi decomposition $P=W \rtimes G$ (as is shown in Lemma 9.7). Let $P^{*}$ be the group generated by $G Q$, then $\mathcal{R}_{u}\left(P^{*}\right)=W^{*}$ and $P^{*} / W^{*}=G$. The group $P^{*}$ defines a connected mixed Shimura datum $\left(P^{*}, \mathcal{X}^{*+}\right)$ with $\mathcal{X}^{*+}=P^{*}(\mathbb{R})^{+} U^{*}(\mathbb{C}) \widetilde{z}$. Now $\widetilde{Z}=H_{\widetilde{Z}}(\mathbb{R})^{+} U_{H_{\widetilde{Z}}}(\mathbb{C}) \widetilde{z} \subset \mathcal{X}^{*+}$. But $\widetilde{Z}$ is Hodge generic in $\mathcal{X}^{+}$by assumption, hence $P=P^{*}$ and $W=W^{*}$. So $W_{H_{\widetilde{Z}}} \triangleleft W$ and hence $W_{H_{\tilde{Z}}} \triangleleft P$.

Use the notation in $\S 2.2$. We are done if we can prove:

$$
\forall u \in U, \forall v \in V, \text { and } \forall g \in G_{H_{\tilde{Z}}},(u, v, 1)(0,0, g)(-u,-v, 1) \in H_{\widetilde{Z}} .
$$


By Corollary 2.14, there exist decompositions

$$
U=U_{N} \oplus U_{N}^{\perp} \quad V=V_{N} \oplus V_{N}^{\perp}
$$

as $G$-modules such that $G_{N}$ acts trivially on $U_{N}^{\perp}$ and $V_{N}^{\perp}$. Now

$$
\begin{aligned}
(u, v, 1)(0,0, g)(-u,-v, 1) & =(u, v, g)(-u,-v, 1) \\
& =(u-g \cdot u, v-g \cdot v, g) \\
& =\left(\left(u_{N}+u_{N}^{\perp}\right)-g \cdot\left(u_{N}+u_{N}^{\perp}\right),\left(v_{N}+v_{N}^{\perp}\right)-g \cdot\left(v_{N}+v_{N}^{\perp}\right), g\right) \\
& =\left(u_{N}-g \cdot u_{N}, v_{N}-g \cdot v_{N}, g\right) \\
& =\left(u_{N}, v_{N}, 1\right)(0,0, g)\left(-u_{N},-v_{N}, 1\right) \in H_{\widetilde{Z}},
\end{aligned}
$$

where the last inclusion follows from Lemma 9.3.

\section{Ax-Lindemann-Weierstrass Part 2: Estimate}

This section is devoted to prove Proposition 9.6. The proof uses essentially the "block family" version of Pila-Wilkie's counting theorem [47, Theorem 3.6].

Keep notation and assumptions as in the last section and denote by $\pi:\left(P, \mathcal{X}^{+}\right) \rightarrow\left(G, \mathcal{X}_{G}^{+}\right)$. The group $G=Z(G)^{\circ} H_{1} \ldots H_{r}$ is an almost direct product, where $H_{i}$ 's are non-trivial simple groups and are normal in $G$. We have a decomposition

$$
\left(G^{\mathrm{ad}}, \mathcal{X}_{G}^{+}\right) \simeq \prod_{i=1}^{r}\left(H_{i}^{\mathrm{ad}}, \mathcal{X}_{H, i}^{+}\right)
$$

by $[40,3.6]$. Let $S_{G}^{\mathrm{ad}}:=\Gamma_{G}^{\mathrm{ad}} \backslash \mathcal{X}_{G}^{+}$. Shrinking $\Gamma_{G}^{\mathrm{ad}}$ if necessary, we may assume $S_{G}^{\mathrm{ad}} \simeq \prod_{i=1}^{r} S_{H, i}$, where $S_{H, i}$ is a connected pure Shimura variety associated with $\left(H_{i}^{\text {ad }}, \mathcal{X}_{H, i}^{+}\right)$.

Without loss of generality we may assume $G_{N}=H_{1} \ldots H_{l}$. It suffices to prove $H_{i}<G_{H_{\widetilde{Z}}}$ for each $i=1, \ldots, l$. The case $l=0$ is trivial, so we assume that $l \geqslant 1$. Define $Q_{i}:=\pi^{-1}\left(H_{i}\right)$.

10.1. Fundamental set and definability. The goal of this subsection is to prove that there exists $\mathcal{F} \subset \mathcal{X}^{+}$a fundamental set for the action of $\Gamma$ on $\mathcal{X}^{+}$such that unif $\left.\right|_{\mathcal{F}}$ is definable.

First of all, by the Reduction Lemma (Lemma 2.12), it suffices to prove the existence of such a fundamental set for $\left(P, \mathcal{X}^{+}\right)$pure and $\left(P, \mathcal{X}^{+}\right)=\left(P_{2 g}, \mathcal{X}_{2 g}^{+}\right)$. The case where $\left(P, \mathcal{X}^{+}\right)$ is pure is guaranteed by Klingler-Ullmo-Yafaev [35, Theorem 4.1]. Now we prove the case $\left(P, \mathcal{X}^{+}\right)=\left(P_{2 g}, \mathcal{X}_{2 g}^{+}\right)$.

We draw the following diagram to make the notation more clear:

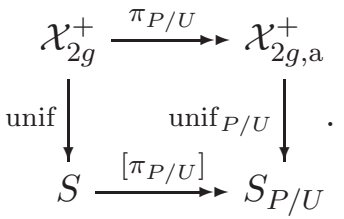

In this case, $\left[\pi_{P / U}\right]: S \rightarrow S_{P / U}$ is an algebraic $\mathbb{G}_{m}$-torsor. By Peterzil-Starchenko [45, Theorem 1.3], there exists a fundamental set $\mathcal{F}_{P / U}$ for the action of $\Gamma / \Gamma_{U}$ on $\mathcal{X}_{2 g \text {,a }}^{+}$such that unif $_{P / U} \mid \mathcal{F}_{P / U}$ is definable (recall that if $g=0$, then $\mathcal{X}_{2 g}^{+}=\mathbb{C}, S=\mathbb{C}^{*}$, unif $=\exp$ and $S_{P / U}$ is a 
point). Let us now construct a fundamental set for the action of $\Gamma$ on $\mathcal{X}_{2 g}^{+}$such that unif $\left.\right|_{\mathcal{F}}$ is definable and $\pi_{P / U}(\mathcal{F})=\mathcal{F}_{P / U}$.

Since any variety over a field is quasi-compact in the Zariski topology, there exists a finite Zariski open covering $\left\{V_{\alpha}\right\}_{\alpha \in \Lambda}$ of $S_{P / U}$ such that $\left.S\right|_{V_{\alpha}} \simeq \mathbb{C}^{*} \times V_{\alpha}$ and these isomorphisms are algebraic. Define $U_{\alpha}:=\left.S\right|_{V_{\alpha}}=\left[\pi_{P / U}\right]^{-1}\left(V_{\alpha}\right)$ for every $\alpha \in \Lambda$. Then we have

$$
\text { unif }\left.\right|_{\text {unif }^{-1}\left(U_{\alpha}\right)}: \text { unif }^{-1}\left(U_{\alpha}\right) \underset{\varphi}{\stackrel{\sim}{\longrightarrow}} U_{2 g}(\mathbb{C}) \times \text { unif }_{P / U}^{-1}\left(V_{\alpha}\right) \rightarrow\left(\mathbb{C}^{*}\right) \times V_{\alpha} \simeq U_{\alpha},
$$

where $\varphi$ is semi-algebraic (Proposition 4.3), the last isomorphism is algebraic and the middle morphism is (exp, unif $P /\left.U\right|_{\text {unif }_{P / U}^{-1}}\left(V_{\alpha}\right)$ ). Let $\mathcal{F}_{U}:=\{s \in \mathbb{C} \mid-1<\mathfrak{R F}(s)<1\}$ and let $\mathcal{F}_{\alpha}:=$ $\varphi^{-1}\left(\mathcal{F}_{U} \times \mathcal{F}_{P / U, \alpha}\right)$. Then unif $\left.\right|_{\mathcal{F}_{\alpha}}$ is definable. Now $\mathcal{F}:=\cup \mathcal{F}_{\alpha}$ (remember that this is a finite union) satisfies the conditions we want.

Now we return to arbitrary $\left(P, \mathcal{X}^{+}\right)$. We have proved the existence of an $\mathcal{F}$ as stated at the beginning of this subsection. Let us choose such an $\mathcal{F}$ more carefully. First of all replace $\mathcal{F}$ by $\gamma \mathcal{F}$ if necessary to make sure $\mathcal{F} \cap \widetilde{Z} \neq \emptyset$. Next define $\mathcal{F}_{G}:=\pi(\mathcal{F}) \subset \mathcal{X}_{G}^{+} \simeq \prod_{i=1}^{r} \mathcal{X}_{H, i}^{+}$. Denote by $q_{i}$ the $i$-th projection and $\mathcal{F}_{H, i}:=q_{i}\left(\mathcal{F}_{G}\right)$. There exist some $\gamma_{1}=1, \ldots, \gamma_{s} \in \Gamma_{G}<\Gamma$ such that $\prod_{i=1}^{r} \mathcal{F}_{H, i} \subset \cup_{j=1}^{s} \gamma_{j} \mathcal{F}_{G}$. Consider

$$
\mathcal{F}^{\prime}:=\left(\bigcup_{j=1}^{s} \gamma_{j} \mathcal{F}\right) \cap \pi^{-1}\left(\prod_{i=1}^{r} \mathcal{F}_{H, i}\right),
$$

then $\mathcal{F}^{\prime}$ is a fundamental set for the action of $\Gamma$ on $\mathcal{X}^{+}$and unif $\left.\right|_{\mathcal{F}^{\prime}}$ is definable. Furthermore, $\pi\left(\mathcal{F}^{\prime}\right)=\prod_{i=1}^{r} \mathcal{F}_{H, i}$ and $\mathcal{F}_{H, i}=q_{i} \pi\left(\mathcal{F}^{\prime}\right)$. We still have $\mathcal{F}^{\prime} \cap \widetilde{Z} \neq \emptyset$ since $\mathcal{F} \subset \mathcal{F}^{\prime}$. Now replace $\mathcal{F}$ by $\mathcal{F}^{\prime}$.

10.2. Counting points and conclusion. We shall work from now on with an $\mathcal{F}$ satisfying the conditions in the last paragraph of the previous subsection. By Lemma 9.5, $\overline{\widetilde{Y}}_{G}=$ $\prod_{i=1}^{l} H_{i}(\mathbb{R})^{+} \widetilde{z}_{G}$. Fix a point $\widetilde{z} \in \mathcal{F} \cap \widetilde{Z}$. Define the following Shimura morphisms for each $i=1, \ldots, l$

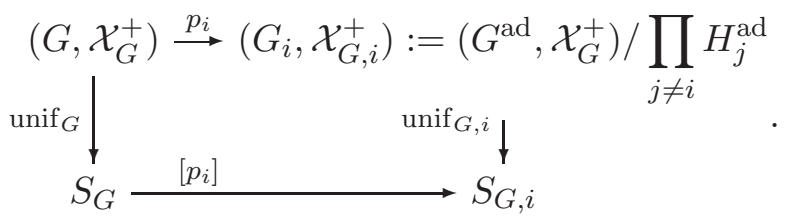

Fix $i \in\{1, \ldots, l\}$. Define $\widetilde{Y}_{G, i}:=p_{i}\left(\widetilde{Y}_{G}\right)=H_{i}^{\text {ad }}(\mathbb{R})^{+} \pi_{i}\left(\widetilde{z}_{G}\right), \widetilde{Z}_{G, i}:=p_{i}\left(\widetilde{Z}_{G}\right)$ and $Y_{G, i}:=\left[p_{i}\right]\left(Y_{G}\right)$, then $\operatorname{unif}_{G, i}\left(\widetilde{Z}_{G, i}\right)$ is Zariski dense in $\overline{Y_{G, i}}$ by Lemma 9.5. If $\operatorname{dim}\left(\widetilde{Z}_{G, i}\right)=0$, then $\widetilde{Z}_{G, i}$ is a finite set of points since it is algebraic. But then $\operatorname{unif}_{G, i}\left(\widetilde{Z}_{G, i}\right)$, and hence $\overline{Y_{G, i}}=\overline{\operatorname{unif}_{G, i}\left(\widetilde{Z}_{G, i}\right)}$ is also a finite set of points. So $\operatorname{dim}\left(Y_{G, i}\right)=0$, which contradicts $\widetilde{Y}_{G, i}=H_{i}^{\text {ad }}(\mathbb{R})^{+} \pi_{i}\left(\widetilde{z}_{G}\right)$. To sum it up, $\operatorname{dim}\left(\widetilde{Z}_{G, i}\right)>0$. For further convenience, we will denote by $\pi_{i}:=p_{i} \circ \pi$.

Take an algebraic curve $C_{G, i} \subset \widetilde{Z}_{G, i}$ passing through $\pi_{i}(\widetilde{z})$. Now $\pi_{i}\left(\widetilde{Z} \cap \pi_{i}^{-1}\left(C_{G, i}\right)\right)=\widetilde{Z}_{G, i} \cap$ $C_{G, i}=C_{G, i}$, and hence there exists an algebraic curve $C \subset \widetilde{Z} \cap \pi_{i}^{-1}\left(C_{G, i}\right)$ passing through $\widetilde{z}$ such that $\operatorname{dim}\left(\pi_{i}(C)\right)=1$.

Let $\mathcal{F}_{G, i}:=p_{i}\left(\mathcal{F}_{G}\right)$, then it is a fundamental set of unif $\sin _{i}$ and $\left.\operatorname{unif}_{G, i}\right|_{\mathcal{F}_{G, i}}$ is definable. We define for any irreducible semi-algebraic subvariety $A\left(\operatorname{resp} . A_{G, i}\right)$ of unif $^{-1}(Y)\left(\operatorname{resp} . \operatorname{unif}_{G, i}^{-1}\left(\overline{Y_{G, i}}\right)\right)$ 
the following sets: define

$$
\begin{gathered}
\Sigma^{(i)}(A):=\left\{g \in Q_{i}(\mathbb{R}) \mid \operatorname{dim}\left(g A \cap \operatorname{unif}^{-1}(Y) \cap \mathcal{F}\right)=\operatorname{dim}(A)\right\} \\
\left(\operatorname{resp.} \Sigma_{G}^{(i)}\left(A_{G, i}\right):=\left\{g \in H_{i}^{\text {ad }}(\mathbb{R}) \mid \operatorname{dim}\left(g A_{G, i} \cap \operatorname{unif}_{G, i}^{-1}\left(\overline{Y_{G, i}}\right) \cap \mathcal{F}_{G, i}\right)=\operatorname{dim}\left(A_{G, i}\right)\right\}\right)
\end{gathered}
$$

and

$$
\begin{gathered}
\Sigma^{\prime(i)}(A):=\left\{g \in Q_{i}(\mathbb{R}) \mid g^{-1} \mathcal{F} \cap A \neq \emptyset\right\} \\
\text { (resp. } \left.\Sigma_{G}^{\prime(i)}\left(A_{G, i}\right):=\left\{g \in H_{i}^{\text {ad }}(\mathbb{R}) \mid g^{-1} \mathcal{F}_{G, i} \cap A_{G, i} \neq \emptyset\right\}\right) .
\end{gathered}
$$

Then $\Sigma^{(i)}(A)$ and $\Sigma_{G}^{(i)}\left(A_{G, i}\right)$ are by definition definable. Let $\Gamma_{G, i}^{\mathrm{ad}}:=p_{i}\left(\Gamma_{G}^{\mathrm{ad}}\right)$.

Lemma 10.1. $\Sigma^{\prime(i)}(A) \cap \Gamma=\Sigma^{(i)}(A) \cap \Gamma\left(\operatorname{resp} . \Sigma_{G}^{\prime(i)}\left(A_{G, i}\right) \cap \Gamma_{G, i}^{\mathrm{ad}}=\Sigma_{G}^{(i)}\left(A_{G, i}\right) \cap \Gamma_{G, i}^{\mathrm{ad}}\right)$.

Proof. The proof, which we include for completeness, is the same as [64, Lemma 5.2]. First of all $\Sigma^{(i)}(A) \cap \Gamma \subset \Sigma^{\prime(i)}(A) \cap \Gamma$ by definition. Conversely for any $\gamma \in \Sigma^{\prime(i)}(A) \cap \Gamma, \gamma^{-1} \mathcal{F} \cap A$ contains an open subspace of $A$ since $\mathcal{F}$ is by choice open in $\mathcal{X}^{+}$. Hence $\gamma A \cap$ unif $^{-1}(Y) \cap \mathcal{F}=\gamma A \cap \mathcal{F}$ contains an open subspace of $\gamma A$ which must be of $\operatorname{dimension} \operatorname{dim}(A)$. Hence $\gamma \in \Sigma^{(i)}(A) \cap \Gamma$. The proof for $A_{G, i}$ is the same.

This lemma implies

$$
\begin{gathered}
\Sigma^{(i)}(C) \cap \Gamma=\Sigma^{\prime(i)}(C) \cap \Gamma \subset \Sigma^{\prime(i)}(\widetilde{Z}) \cap \Gamma=\Sigma^{(i)}(\widetilde{Z}) \cap \Gamma \\
\left(\text { resp. } \Sigma_{G}^{(i)}\left(C_{G, i}\right) \cap \Gamma_{G, i}^{\mathrm{ad}}=\Sigma_{G}^{\prime(i)}\left(C_{G_{i}}\right) \cap \Gamma_{G, i}^{\mathrm{ad}} \subset \Sigma_{G}^{\prime(i)}\left(\overline{\widetilde{Z}_{G, i}}\right) \cap \Gamma_{G, i}^{\mathrm{ad}}=\Sigma^{(i)}\left(\overline{\widetilde{Z}_{G, i}}\right) \cap \Gamma_{G, i}^{\mathrm{ad}}\right)
\end{gathered} .
$$

Lemma 10.2. $\pi_{i}\left(\Gamma \cap \Sigma^{(i)}(C)\right)=\Gamma_{G, i}^{\mathrm{ad}} \cap \Sigma_{G}^{(i)}\left(C_{G, i}\right)$.

Proof. By Lemma 10.1, it suffices to prove $\pi_{i}\left(\Gamma \cap \Sigma^{\prime(i)}(C)\right)=\Gamma_{G, i}^{\mathrm{ad}} \cap \Sigma_{G}^{\prime(i)}\left(C_{G, i}\right)$. The inclusion $\subset$ is clear by definition. For the other inclusion, $\forall \gamma_{G, i} \in \Gamma_{G, i}^{\mathrm{ad}} \cap \Sigma_{G}^{\prime(i)}\left(C_{G, i}\right), \exists c_{G, i} \in C_{G, i}$ such that $\gamma_{G, i} \cdot c_{G, i} \in \mathcal{F}_{G, i}$.

Take a point $c \in C$ such that $\pi_{i}(c)=c_{G, i}$ and define $c_{G}:=\pi(c) \in \mathcal{X}_{G}^{+}$. Suppose that under the decomposition

$$
\left(G^{\mathrm{ad}}, \mathcal{X}_{G}^{+}\right) \simeq \prod_{i=1}^{r}\left(H_{i}^{\mathrm{ad}}, \mathcal{X}_{H, i}^{+}\right)
$$

of $[40,3.6], c_{G}=\left(c_{G, 1}, \ldots, c_{G, r}\right)$. Then by choice of $\mathcal{F}_{G}$, there exists $\gamma_{G}^{\prime} \in \Gamma_{G}^{\mathrm{ad}}$ whose $i$-th coordinate is precisely the $\gamma_{G, i}$ in the last paragraph such that $\gamma_{G}^{\prime} \cdot c_{G} \in \mathcal{F}_{G}$.

Let $\gamma_{G} \in \Gamma_{G}$ be such that its image under $\Gamma_{G} \rightarrow \Gamma_{G}^{\mathrm{ad}}$ is $\gamma_{G}^{\prime}$, then $\gamma_{G} \cdot c \in \pi^{-1}\left(\mathcal{F}_{G}\right)$. Therefore there exist $\gamma_{V} \in \Gamma_{V}, \gamma_{U} \in \Gamma_{U}$ such that $\left(\gamma_{U}, \gamma_{V}, \gamma_{G}\right) c \in \mathcal{F}$. Denote by $\gamma=\left(\gamma_{U}, \gamma_{V}, \gamma_{G}\right)$, then $\gamma \in \Gamma \cap \Sigma^{\prime(i)}(C)$ and $\pi_{i}(\gamma)=\gamma_{G, i}$.

For $T>0$, define

$$
\Theta_{G}^{(i)}\left(C_{G, i}, T\right):=\left\{\gamma_{G} \in \Gamma_{G, i}^{\mathrm{ad}} \cap \Sigma_{G}^{(i)}\left(C_{G, i}\right) \mid H\left(\gamma_{G}\right) \leqslant T\right\} .
$$

Proposition 10.3. There exists a constant $\delta>0$ s.t. for all $T \gg 0,\left|\Theta_{G}^{(i)}\left(C_{G, i}, T\right)\right| \geqslant T^{\delta}$.

Proof. This follows directly from [35, Theorem 1.3] applied to $\left(\left(G_{i}, \mathcal{X}_{G, i}^{+}\right), S_{G, i}, \bar{Z}_{G, i}\right)$. 
Let us prove how these facts imply $H_{i}<G_{H_{\tilde{Z}}}$.

Take a faithful representation $G^{\text {ad }} \hookrightarrow \mathrm{GL}_{n}$ which sends $\Gamma_{G}^{\text {ad }}$ to $\mathrm{GL}_{n}(\mathbb{Z})$. Consider the definable set $\Sigma_{G}^{(i)}\left(C_{G, i}\right)$. By the theorem of Pila-Wilkie ([47, Theorem 3.6]), there exist $J=J(\delta)$ definable block families

$$
B^{j} \subset \Sigma_{G}^{(i)}\left(C_{G, i}\right) \times \mathbb{R}^{l}, \quad j=1, \ldots, J
$$

and $c=c(\delta)>0$ such that for all $T \gg 0, \Theta_{G}^{(i)}\left(C_{G, i}, T^{1 / 2 n}\right)$ is contained in the union of at most $c T^{\delta / 4 n}$ definable blocks of the form $B_{y}^{j}\left(y \in \mathbb{R}^{l}\right)$. By Proposition 10.3, there exist a $j \in\{1, \ldots, J\}$ and a block $B_{G, i}:=B_{y_{0}}^{j}$ of $\Sigma_{G}^{(i)}\left(C_{G, i}\right)$ containing at least $T^{\delta / 4 n}$ elements of $\Theta_{G}^{(i)}\left(C_{G, i}, T^{1 / 2 n}\right)$.

Let $\Sigma^{(i)}:=\Sigma^{(i)}(C) \cap \Sigma^{(i)}(\widetilde{Z})$, which is by definition a definable set. Consider $X^{j}:=\left(\pi_{i} \times\right.$ $\left.1_{\mathbb{R}^{l}}\right)^{-1}\left(B^{j}\right) \cap\left(\Sigma^{(i)} \times \mathbb{R}^{l}\right)$, which is a definable family since $\pi_{i}$ is algebraic.

By [23, Ch. 3, 3.6], there exists a number $n_{0}>0$ such that each fibre $X_{y}^{j}$ has at most $n_{0}$ connected components. So the definable set $\pi_{i}^{-1}\left(B_{G, i}\right) \cap \Sigma^{(i)}$ has at most $n_{0}$ connected components. Now

$$
\pi_{i}\left(\pi_{i}^{-1}\left(B_{G, i}\right) \cap \Sigma^{(i)} \cap \Gamma\right)=B_{G, i} \cap \pi_{i}\left(\Sigma^{(i)}(C) \cap \Gamma\right)=B_{G, i} \cap \Sigma_{G}^{(i)}\left(C_{G, i}\right) \cap \Gamma_{G, i}^{\mathrm{ad}}=B_{G, i} \cap \Gamma_{G, i}^{\mathrm{ad}}
$$

by (10.1) and Lemma 10.2. So there exists a connected component $B$ of $\pi_{i}^{-1}\left(B_{G, i}\right) \cap \Sigma^{(i)}$ such that $\pi_{i}(B \cap \Gamma)$ contains at least $T^{\delta / 4 n} / n_{0}$ elements of $\Theta_{G}^{(i)}\left(C_{G, i}, T^{1 / 2 n}\right)$.

We have $B \widetilde{Z} \subset$ unif $^{-1}(Y)$ since $\Sigma^{(i)}(\widetilde{Z}) \widetilde{Z} \subset$ unif $^{-1}(Y)$ by analytic continuation, and $\widetilde{Z} \subset$ $\sigma^{-1} B \widetilde{Z}$ for any $\sigma \in B \cap \Gamma$. But $B$ is connected, and therefore $\sigma^{-1} B \widetilde{Z}=\widetilde{Z}$ by maximality of $\widetilde{Z}$ and [50, Lemma 4.1]. So $\forall \sigma \in B \cap \Gamma$,

$$
B \subset \sigma \operatorname{Stab}_{Q_{i}(\mathbb{R})}(\widetilde{Z}) .
$$

Fix a $\gamma_{0} \in B \cap \Gamma$ such that $\pi_{i}\left(\gamma_{0}\right) \in \Theta_{G}^{(i)}\left(C_{G, i}, T^{1 / 2 n}\right)$. We have already shown that $\pi_{i}(B \cap \Gamma)$ contains at least $T^{\delta / 4 n} / n_{0}$ elements of $\Theta_{G}^{(i)}\left(C_{G, i}, T^{1 / 2 n}\right)$. For any $\gamma_{G, i}^{\prime} \in \pi_{i}(B \cap \Gamma) \cap \Theta_{G}^{(i)}\left(C_{G, i}, T^{1 / 2 n}\right)$, let $\gamma^{\prime}$ be one of its pre-images in $B \cap \Gamma$. Then $\gamma:=\gamma^{\prime-1} \gamma_{0}$ is an element of $\Gamma \cap \operatorname{Stab}_{Q_{i}(\mathbb{R})}(\widetilde{Z})=$ $\Gamma_{\widetilde{Z}} \cap Q_{i}(\mathbb{R})$ such that $H\left(\pi_{i}(\gamma)\right) \ll T^{1 / 2}$. Therefore for $T \gg 0, \pi_{i}\left(\Gamma_{\widetilde{Z}}\right) \cap H_{i}^{\text {ad }}(\mathbb{R})$ contains at least $T^{\delta / 4 n} / n_{0}$ elements $\gamma_{G, i}$ such that $H\left(\gamma_{G, i}\right) \leqslant T$. Hence $\operatorname{dim}\left(\pi_{i}\left(H_{\widetilde{Z}}\right) \cap H_{i}^{\text {ad }}\right)>0$ since $\pi_{i}\left(H_{\widetilde{Z}}\right) \cap H_{i}^{\text {ad }}$ contains infinitely many rational points. But $\pi_{i}\left(H_{\widetilde{Z}}\right)=p_{i} \pi\left(H_{\widetilde{Z}}\right)=p_{i}\left(G_{H_{\widetilde{Z}}}\right)$ by definition. So $H_{i}^{\text {ad }}<p_{i}\left(G_{H_{\widetilde{Z}}}\right)$ since $H_{i}^{\text {ad }}$ is simple and $p_{i}\left(G_{H_{\widetilde{Z}}}\right) \cap H_{i}^{\text {ad }} \triangleleft H_{i}^{\text {ad }}$ by Corollary 9.4.

As a normal subgroup of $G_{N}, G_{H_{\tilde{Z}}}$ is the almost direct product of some $H_{j}$ 's $(j=1, \ldots, l)$. So $H_{i}^{\text {ad }}<p_{i}\left(G_{H_{\tilde{Z}}}\right)$ implies $H_{i}<G_{H_{\tilde{Z}}}$. Now we are done.

Remark 10.4. In the proof of the pure case by Klingler-Ullmo-Yafaev [35], it suffices to use a non-family version of Pila-Wilkie ([35, Theorem 6.1]). However this is not enough for our proof, since otherwise the $n_{0}$ would depend on $T$. Hence it is important to use a family version of Pila-Wilkie ([47, Theorem 3.6]).

\section{Ax-Lindemann-Weierstrass Part 3: The unipotent part}

We prove in this section Theorem 7.6. We use the same notation as the first paragraph of $\S 7$ and $\S 7.3$. Assume $\operatorname{dim}_{\mathbb{C}} T=m$ and $\operatorname{dim}_{\mathbb{C}} A=n$. 
Proof of Theorem 7.6. First of all we may assume that $\widetilde{Z}$ is of positive dimension since every point is a weakly special subvariety of dimension 0 . For any fundamental set $\mathcal{F}$ of the action of $\Gamma_{W}$ on $W(\mathbb{R}) U(\mathbb{C})$, define

$$
\Sigma(\widetilde{Z}):=\left\{g \in W(\mathbb{R}) \mid \operatorname{dim}\left(g \widetilde{Z} \cap \operatorname{unif}^{-1}(Y) \cap \mathcal{F}\right)=\operatorname{dim}(\widetilde{Z})\right\}
$$

and

$$
\Sigma^{\prime}(\widetilde{Z}):=\left\{g \in W(\mathbb{R}) \mid g^{-1} \mathcal{F} \cap \widetilde{Z} \neq \emptyset\right\}
$$

then by Lemma 10.1,

$$
\Sigma(\widetilde{Z}) \cap \Gamma_{W}=\Sigma^{\prime}(\widetilde{Z}) \cap \Gamma_{W}
$$

Let $\Gamma_{U}:=\Gamma \cap U(\mathbb{Q})$ and let $\Gamma_{V}:=\Gamma_{W} / \Gamma_{U}$.

Case $i: E=A$. This is [52, Theorem 2.1 and pp9 Remark 1]. A proof can be found in Appendix. In this case, $W=V$ and $\Gamma_{V}=\oplus_{i=1}^{2 n} \mathbb{Z} e_{i} \subset \operatorname{Lie}(A)=\mathbb{C}^{n}=\mathbb{R}^{2 n}$ is a lattice. Denote by unif: $\operatorname{Lie}(A) \rightarrow A$. Let $\mathcal{F}_{V}:=\Sigma_{i=1}^{2 n}(-1,1) e_{i}$, then $\mathcal{F}_{V}$ is a fundamental set for the action of $\Gamma_{V}$ on $\operatorname{Lie}(A)$ such that unif $\left.\right|_{\mathcal{F}_{V}}$ is definable.

Case ii : $E=T$. This is a consequence of Ax's theorem [5] [42, Corollary 3.6]. A proof of this can be found in Appendix. In this case, $W=U$. Let $\mathcal{F}_{U}:=\{s \in \mathbb{C} \mid-1<\mathfrak{R F}(s)<1\}^{m}$, then $\mathcal{F}_{U}$ is a fundamental set for the action of $\Gamma_{U}$ on $U(\mathbb{C})$ such that unif $\left.\right|_{\mathcal{F}_{U}}$ is definable. map

Case iii : general E. Unlike the rest of the paper, the symbol $\pi$ in this section denotes the

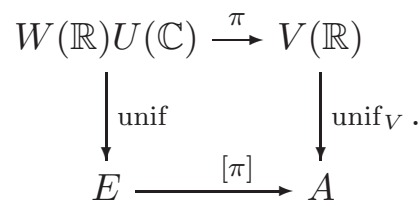

Take $\mathcal{F}_{V} \subset V(\mathbb{R})$ any fundamental set for the action of $\Gamma_{V}$ on $V(\mathbb{R})$ such that unif $\left._{V}\right|_{\mathcal{F}_{V}}$ is definable. We claim that:

There exists a fundamental set $\mathcal{F}$ for the action of $\Gamma_{W}$ on $W(\mathbb{R}) U(\mathbb{C})$ such that unif $\left.\right|_{\mathcal{F}}$ is definable and $\pi(\mathcal{F})=\mathcal{F}_{V}$.

By Reduction Lemma (Lemma 2.12), it suffices to prove this for $E=E_{1} \times_{A} \ldots \times_{A} E_{m}$ where $E_{i}$ 's are $\mathbb{G}_{m}$-torsors over $A$. But then it suffices to prove for the case $m=1$. For this case, the proof is similar to $§ 10.1$.

Let $Y_{0}$ be the minimal closed irreducible subvariety of $E$ such that $\widetilde{Z} \subset \operatorname{unif}^{-1}\left(Y_{0}\right)$, then $\widetilde{Z}$ is maximal irreducible algebraic in $\operatorname{unif}^{-1}\left(Y_{0}\right)$. Hence we may assume that $Y=Y_{0}$. Let $N$ be the connected algebraic monodromy group of $Y^{\mathrm{sm}}$ and let $V_{N}:=(N \cap W) /(N \cap U)$. Let $\widetilde{Y}$ be the complex analytic irreducible component of unif ${ }^{-1}(Y)$ which contains $\widetilde{Z}$. For further convenience, we will denote by $\widetilde{Z}_{V}:=\pi(\widetilde{Z}), \widetilde{Y}_{V}:=\pi(\widetilde{Y})$ and $Y_{V}:=[\pi](Y)$.

Repeating the proof of Lemma 9.5 (but using the conclusion of Case $i$ instead of [35, Theorem 1.1]), we get that $\overline{\widetilde{Y}_{V}}=V_{N}(\mathbb{R})+\widetilde{z}_{V}$ for some $\widetilde{z}_{V} \in \widetilde{Z}_{V}$ is weakly special, and $\overline{\text { unif }_{V}\left(\widetilde{Z}_{V}\right)}=\overline{Y_{V}}$. Remark that by GAGA, these closures could be taken in the complex analytic topology (i.e. the topology whose closed sets are complex analytic sets) or the Zariski topology. If $V_{N}$ is trivial, then we are actually in the situation of Case $i$, and therefore $\widetilde{Z}$ is weakly special. From now 
on, suppose that $\operatorname{dim}\left(V_{N}\right)>0$. Replace $S$ by its smallest special subvariety containing $Y_{0}$, then $N \triangleleft P$ by Theorem 3.7. Hence $V_{N}$ is a $G=\mathrm{MT}(b)$-submodule of $V$.

Define $W_{0}:=\left({\overline{\Gamma_{W} \cap \operatorname{Stab}_{W(\mathbb{R}) U(\mathbb{C})}(\widetilde{Z}}}^{\text {Zar }}\right)^{\circ}, U_{0}:=W_{0} \cap U$ and $V_{0}:=\pi\left(W_{0}\right)=W_{0} / U_{0}$. The proof is somehow technical, so we will divide it into several steps.

Step I. Let $V^{\dagger}$ be the smallest subgroup of $V_{N}$ such that $\widetilde{Z}_{V} \subset V^{\dagger}(\mathbb{R})+\widetilde{z}_{V}$. In Step I, we will prove $V^{\dagger}<V_{0}$.

Step $I(i)$. We know that $A=\Gamma_{V} \backslash V(\mathbb{R})$ and $V(\mathbb{Q}) \simeq \Gamma_{V} \otimes_{\mathbb{Z}} \mathbb{Q}$. Consider any $\mathbb{Q}$-quotient group $V^{\prime}$ of $V$ of dimension 1

$$
p^{\prime}: V \rightarrow V^{\prime}
$$

such that $\operatorname{dim}\left(p^{\prime}\left(V^{\dagger}\right)\right)=1$. By abuse of notation, we shall denote its induced map $V(\mathbb{R}) \rightarrow V^{\prime}(\mathbb{R})$ also by $p^{\prime}$. Now let $\Gamma_{V^{\prime}}:=p^{\prime}\left(\Gamma_{V}\right)$, then $\Gamma_{V^{\prime}} \simeq \mathbb{Z}$ since $p^{\prime}$ is defined over $\mathbb{Q}$. Write $\Gamma_{V^{\prime}}=\mathbb{Z} e^{\prime}$, and let $\mathcal{F}_{V^{\prime}}:=(-1,1) e^{\prime}$. Then $\mathcal{F}_{V^{\prime}}$ is a fundamental set for the action of $\Gamma_{V^{\prime}}$ on $V^{\prime}(\mathbb{R})$. Define $A^{\prime}=\Gamma_{V^{\prime}} \backslash V^{\prime}(\mathbb{R}) \simeq \mathbb{Z} \backslash \mathbb{R}$, unif $V_{V^{\prime}}: V^{\prime}(\mathbb{R}) \rightarrow A^{\prime}$ the uniformization and $\left[p^{\prime}\right]: A \rightarrow A^{\prime}$ the map induced by $p^{\prime}$. Then unif $\left.{ }_{V^{\prime}}\right|_{\mathcal{F}_{V^{\prime}}}$ is definable (even in $\mathbb{R}_{a n}$ ). Define $Y_{V^{\prime}}:=\left[p^{\prime}\right]\left(Y_{V}\right)$ and $\widetilde{Y}_{V^{\prime}}:=p^{\prime}\left(\widetilde{Y}_{V}\right)$.

Let $V^{\prime \prime}:=\operatorname{Ker}\left(p^{\prime}\right)$. The exact sequence of free $\mathbb{Z}$-modules

$$
1 \rightarrow \Gamma_{V^{\prime \prime}}:=\Gamma_{V} \cap V^{\prime \prime}(\mathbb{Q}) \simeq \mathbb{Z}^{2 n-1} \rightarrow \Gamma_{V} \simeq \mathbb{Z}^{2 n} \rightarrow \Gamma_{V^{\prime}} \simeq \mathbb{Z} \rightarrow 1
$$

splits, and hence $\Gamma_{V} \simeq \Gamma_{V^{\prime \prime}} \oplus \Gamma_{V^{\prime}}$. This induces $V \simeq V^{\prime \prime} \oplus V^{\prime}$. Write $\Gamma_{V^{\prime \prime}}=\sum_{i=2}^{2 n} \mathbb{Z} e_{i}^{\prime \prime}$ and take $\mathcal{F}_{V^{\prime \prime}}:=\sum_{i=2}^{n}(-1,1) e_{i}^{\prime \prime}$. Define $\mathcal{F}_{V}:=\mathcal{F}_{V^{\prime \prime}} \oplus \mathcal{F}_{V^{\prime}}$. Then $\mathcal{F}_{V}$ is a fundamental set for the action of $\Gamma_{V}$ on $V(\mathbb{R})$ such that unif $\left.\right|_{V} \mathcal{F}_{V}$ is definable (even in $\mathbb{R}_{a n}$ ). Define $\mathcal{F}$ as in (11.3).

Since $p\left(V^{\dagger}\right)=V^{\prime}$ by choice of $V^{\prime}, \operatorname{dim}_{\mathbb{R}} p^{\prime}\left(\widetilde{Z}_{V}\right)>0$ by minimality of $V^{\dagger}$. Hence $p^{\prime}\left(\widetilde{Z}_{V}\right)=V^{\prime}(\mathbb{R})$ since $p^{\prime}\left(\widetilde{Z}_{V}\right)$ is connected.

Remark 11.1. If we only request $\left(V^{\prime}, p^{\prime}\right)$ to satisfy $p^{\prime}\left(V_{N}\right)=1$, then we do not know whether $\operatorname{dim}_{\mathbb{R}}\left(p^{\prime}\left(\widetilde{Z}_{V}\right)\right)>0$. This is because we are considering the real analytic topology (i.e. the topology whose closed sets are real analytic sets) on $A^{\prime}$ and the complex analytic topology (i.e. the topology whose closed sets are complex analytic sets) on $A$, and hence $\overline{\text { unif }_{V}\left(\widetilde{Z}_{V}\right)}=\overline{Y_{V}}$ does NOT imply $\overline{\text { unif }_{V^{\prime}}\left(\widetilde{Z}_{V^{\prime}}\right)}=\overline{Y_{V^{\prime}}}$. To overcome this problem, we introduce the seemingly strange subgroup $V^{\dagger}$ of $V_{N}$. We will prove (Step II) that $V_{0}$ is a $\mathrm{MT}(b)$-module with the help of $V^{\dagger}$. Then we prove the comparable result of Theorem 9.8(1) for the unipotent part in Step III.

Let $C$ be an $\mathbb{R}$-algebraic subvariety of $\widetilde{Z}$ of $\mathbb{R}$-dimension 1 such that $p^{\prime} \pi(C)=V^{\prime}(\mathbb{R})$. Define furthermore

$$
\Sigma(C):=\left\{g \in W(\mathbb{R}) \mid \operatorname{dim}_{\mathbb{R}}\left(g C \cap \operatorname{unif}^{-1}(Y) \cap \mathcal{F}\right)=1\right\}
$$

and

$$
\Sigma^{\prime}(C):=\left\{g \in W(\mathbb{R}) \mid g^{-1} \mathcal{F} \cap C \neq \emptyset\right\} .
$$

The set $\Sigma(C)$ is by definition definable. By Lemma 10.1,

$$
\Sigma^{\prime}(C) \cap \Gamma_{W}=\Sigma(C) \cap \Gamma_{W}
$$

For $M>0$, define

$$
\Theta_{V^{\prime}}\left(V^{\prime}(\mathbb{R}), M\right)=\left\{\gamma_{V^{\prime}} \in \Gamma_{V^{\prime}} \mid H\left(\gamma_{V^{\prime}}\right) \leqslant M\right\} .
$$


Then

$$
\left|\Theta_{V^{\prime}}\left(V^{\prime}(\mathbb{R}), M\right)\right| \gg M
$$

Step I(ii) is quite similar to the end of $\S 10$. Consider the definable set $V^{\prime}(\mathbb{R})$. By the theorem of Pila-Wilkie ([47, Theorem 3.6]), there exist $J$ definable block families

$$
B^{j} \subset V^{\prime}(\mathbb{R}) \times \mathbb{R}^{l}, \quad j=1, \ldots, J
$$

and $c>0$ such that for all $M \gg 0, \Theta_{V^{\prime}}\left(V^{\prime}(\mathbb{R}), M^{1 / 4}\right)$ is contained in the union of at most $c M^{\delta / 8}$ definable blocks of the form $B_{y}^{j}\left(y \in \mathbb{R}^{l}\right)$. By (11.5), there exist a $j \in\{1, \ldots, J\}$ and a block $B_{V^{\prime}}:=B_{y_{0}}^{j}$ of $V^{\prime}(\mathbb{R})$ containing at least $M^{\delta / 8}$ elements of $\Theta_{V^{\prime}}\left(V^{\prime}(\mathbb{R}), M^{1 / 4}\right)$.

Let $\Sigma:=\Sigma(C) \cap \Sigma(\widetilde{Z})$, which is by definition a definable set. Consider $X^{j}:=\left(\left(p^{\prime} \pi\right) \times\right.$ $\left.1_{\mathbb{R}^{l}}\right)^{-1}\left(B^{j}\right) \cap\left(\Sigma \times \mathbb{R}^{l}\right)$, which is a definable family since $p^{\prime} \pi$ is $\mathbb{R}$-algebraic.

By [23, Ch. 3, 3.6], there exists a number $n_{0}>0$ such that each fibre $X_{y}^{j}$ has at most $n_{0}$ connected components. So the definable set $\pi^{-1}\left(B_{V^{\prime}}\right) \cap \Sigma$ has at most $n_{0}$ connected components. Now

$$
p^{\prime} \pi\left(\left(p^{\prime} \pi\right)^{-1}\left(B_{V^{\prime}}\right) \cap \Sigma \cap \Gamma_{W}\right)=B_{V^{\prime}} \cap p^{\prime} \pi\left(\Sigma(C) \cap \Gamma_{W}\right)=B_{V^{\prime}} \cap\left(V^{\prime}(\mathbb{R}) \cap \Gamma_{V^{\prime}}\right)=B_{V^{\prime}} \cap \Gamma_{V^{\prime}}
$$

by (11.1), (11.4) and the choice of $\mathcal{F}$ (remember that $\Gamma_{V}=\Gamma_{V^{\prime \prime}} \oplus \Gamma_{V^{\prime}}$ and $\mathcal{F}_{V}=\mathcal{F}_{V^{\prime \prime}} \oplus \mathcal{F}_{V^{\prime}}$ ). So there exists a connected component $B$ of $\left(p^{\prime} \pi\right)^{-1}\left(B_{V^{\prime}}\right) \cap \Sigma$ such that $p^{\prime} \pi\left(B \cap \Gamma_{W}\right)$ contains at least $M^{\delta / 8} / n_{0}$ elements of $\Theta_{V^{\prime}}\left(V^{\prime}(\mathbb{R}), M^{1 / 4}\right)$.

We have $B \widetilde{Z} \subset$ unif $^{-1}(Y)$ since $B \subset \Sigma(\widetilde{Z})$ by (complex) analytic continuation, and $\widetilde{Z} \subset$ $\sigma_{W}^{-1} B \widetilde{Z}$ for any $\sigma_{W} \in B \cap \Gamma_{W}$. But $B$ is connected, and therefore $\sigma_{W}^{-1} B \widetilde{Z}=\widetilde{Z}$ by maximality of $\widetilde{Z}$ and [50, Lemma 4.1]. So

$$
B \subset \sigma_{W} \operatorname{Stab}_{W(\mathbb{R})}(\widetilde{Z}) .
$$

Fix a $\sigma_{W} \in B \cap \Gamma_{W}$ such that $p^{\prime} \pi\left(\sigma_{W}\right) \in \Theta_{V^{\prime}}\left(V^{\prime}(\mathbb{R}), M^{1 / 4}\right)$. We have shown that $p^{\prime} \pi(B \cap$ $\left.\Gamma_{W}\right)$ contains at least $M^{\delta / 8} / n_{0}$ elements of $\Theta_{V^{\prime}}\left(V^{\prime}(\mathbb{R}), M^{1 / 4}\right)$. For any $\sigma_{V^{\prime}} \in p^{\prime} \pi(B \cap \Gamma) \cap$ $\Theta_{V^{\prime}}\left(V^{\prime}(\mathbb{R}), M^{1 / 4}\right)$, let $\sigma_{W}^{\prime}$ be one of its pre-images in $B \cap \Gamma_{W}$. Then $\gamma_{W}:=\sigma_{W}^{-1} \sigma_{W}^{\prime}$ is an element of $\Gamma_{W} \cap \operatorname{Stab}_{W(\mathbb{R})}(\widetilde{Z})$ and $H\left(p^{\prime} \pi\left(\gamma_{W}\right)\right) \ll M^{1 / 2}$. Therefore for $M \gg 0, p^{\prime} \pi\left(\Gamma_{W} \cap \operatorname{Stab}_{W(\mathbb{R})}(\widetilde{Z})\right)$ contains at least $M^{\delta / 8} / n_{0}$ elements $\gamma_{V^{\prime}}$ such that $H\left(\gamma_{V^{\prime}}\right) \leqslant M$. Therefore $\operatorname{dim}\left(p^{\prime} \pi\left(W_{0}\right)\right)>0$ since it is an infinite set. So $p^{\prime} \pi\left(W_{0}\right)=V^{\prime}$ since $\operatorname{dim}\left(V^{\prime}\right)=1$. But $V^{\prime}$ is an arbitrary 1dimensional quotient of $V$ such that $p^{\prime}\left(V^{\dagger}\right)=V^{\prime}$. Therefore $V^{\dagger}<\pi\left(W_{0}\right)=V_{0}$.

Step II. We prove in this step that $V_{0}$ is a MT(b)-module. This implies that $W_{0}$ is a $\mathrm{MT}(b)$ subgroup of $W$ by Proposition 2.6(2).

By definition of $V^{\dagger}, \widetilde{Z}_{V} \subset V^{\dagger}(\mathbb{R})+\widetilde{z}_{V}$. By definition of $V_{0}, V_{0}(\mathbb{R})+\widetilde{z}_{V} \subset \widetilde{Z}_{V}$. Now the conclusion of Step $I$ implies $V_{0}=V^{\dagger}$ and $\widetilde{Z}_{V}=V_{0}(\mathbb{R})+\widetilde{z}_{V}$. However $\widetilde{Z}_{V}$ is complex, so $V_{0}(\mathbb{R})$ is a complex subspace of $V(\mathbb{R})$. Therefore by considering the complex structure of $V(\mathbb{R})$, we get that $V_{0}(\mathbb{R})$ is a $\operatorname{MT}(b)(\mathbb{R})$-module. So $V_{0}$ is a $\operatorname{MT}(b)$-module.

Step III. can be seen as an analogue to the proof of Theorem 9.8(1). Consider a fibre of $\widetilde{Z}$ over a point $v \in \pi(\widetilde{Z})$ such that $\pi: W(\mathbb{C}) / F_{b}^{0} W(\mathbb{C}) \rightarrow \operatorname{Lie}(A)$ is flat at $v$ (such a point exists by generic flatness). Let $\widetilde{W}$ be an irreducible algebraic component of $\widetilde{Z}_{v}$ such that $\operatorname{dim}\left(\widetilde{Z}_{v}\right)=\operatorname{dim}(\widetilde{W})$, then since $\pi$ is flat at $v$,

$$
\operatorname{dim}(\widetilde{Z})=\operatorname{dim}(\pi(\widetilde{Z}))+\operatorname{dim}\left(\widetilde{Z}_{v}\right)=\operatorname{dim}(\pi(\widetilde{Z}))+\operatorname{dim}(\widetilde{W}) .
$$


Consider the set $\widetilde{F}:=W_{0}(\mathbb{R}) U_{0}(\mathbb{C}) \widetilde{W}$. It is semi-algebraic. The fact $\widetilde{W} \subset \widetilde{Z}$ implies that $\widetilde{F} \subset$ $\widetilde{Z}$. So by $\left[50\right.$, Lemma 4.1], there exists an irreducible algebraic subvariety of $W(\mathbb{C}) / F_{b}^{0} W(\mathbb{C})$, say $\widetilde{F}_{\text {alg, }}$, which contains $\widetilde{F}$ and is contained in $\widetilde{Z}$. Since

$$
\pi(\widetilde{F})=\pi\left(W_{0}\right)(\mathbb{R})+v=\overline{\pi(\widetilde{Z})}
$$

and every fiber of $\left.\pi\right|_{\widetilde{F}_{\text {alg }}}$ has $\mathbb{R}$-dimension at least $\operatorname{dim}_{\mathbb{R}}(\widetilde{W})$, we have

$$
\operatorname{dim}\left(\widetilde{F}_{\text {alg }}\right) \geqslant \operatorname{dim}(\pi(\widetilde{F}))+\operatorname{dim}(\widetilde{W})=\operatorname{dim}(\pi(\widetilde{Z}))+\operatorname{dim}(\widetilde{W})=\operatorname{dim}(\widetilde{Z}) .
$$

So $\widetilde{F}=\widetilde{Z}$ since $\widetilde{Z}$ is irreducible. In other words, $\widetilde{Z}=W_{0}(\mathbb{R}) U_{0}(\mathbb{C}) \widetilde{Z}_{v}$ and $\widetilde{Z}_{v}$ is irreducible for any $v \in \pi(\widetilde{Z})$.

Next for any $v \in \pi(\widetilde{Z})$, let $\widetilde{W}^{\prime}$ be an irreducible algebraic subvariety which contains $\widetilde{Z}_{v}$ and is contained in unif ${ }^{-1}(Y)_{v}$, maximal for these properties. Then $\widetilde{W}^{\prime}$ is weakly special by Case ii. Consider $\widetilde{F}^{\prime}:=W_{0}(\mathbb{R}) U_{0}(\mathbb{C}) \widetilde{W}^{\prime}$. Let $\widetilde{Y}$ be the irreducible component of unif ${ }^{-1}(Y)$ which contains $\widetilde{Z}$, then $\widetilde{W^{\prime}} \subset \widetilde{Y}$ and so $\widetilde{F}^{\prime} \subset \widetilde{Y}$ by Lemma 9.2. But $\widetilde{F}^{\prime}$ is semi-algebraic, and hence by $\left[50\right.$, Lemma 4.1] there exists an irreducible algebraic subvariety of $W(\mathbb{C}) / F_{b}^{0} W(\mathbb{C})$, say $\widetilde{F}_{\text {alg }}^{\prime}$, which contains $\widetilde{F}^{\prime}$ and is contained in $\widetilde{Y}$. So $\widetilde{Z}=W_{0}(\mathbb{R}) U_{0}(\mathbb{C}) \widetilde{Z}_{v} \subset \widetilde{F}_{\text {alg }}^{\prime} \subset \operatorname{unif}^{-1}(Y)$, and hence $\widetilde{Z}=\widetilde{F}_{\text {alg }}^{\prime}=\widetilde{F}^{\prime}$ by the maximality of $\widetilde{Z}$. So $\widetilde{Z}_{v}=\widetilde{W}^{\prime}$, i.e.

For any $v \in \pi(\widetilde{Z}), \widetilde{Z}_{v}$ is a maximal irreducible algebraic subvariety of $W(\mathbb{C}) / F^{0} W(\mathbb{C})$ contained in unif ${ }^{-1}(Y)_{v}$.

Now that $\widetilde{Z}_{v}=\widetilde{W}^{\prime}$ is weakly special, we can write $\widetilde{Z}_{v}=U^{\prime}(\mathbb{C})+\widetilde{z}$ with $U^{\prime}<U$ and $\widetilde{z} \in \widetilde{Z}_{v}$. Then $U_{0}<U^{\prime}$. The product $W^{\prime}:=W_{0} U^{\prime}$ is a subgroup of $W$, and hence

$$
\widetilde{Z}=W_{0}(\mathbb{R}) U_{0}(\mathbb{C}) \widetilde{Z}_{v}=W_{0}(\mathbb{R}) U^{\prime}(\mathbb{C}) \widetilde{z}=W^{\prime}(\mathbb{R}) U^{\prime}(\mathbb{C}) \widetilde{z} .
$$

So $W_{0}=W^{\prime}$ and $U_{0}=U^{\prime}$. In other words,

$$
\widetilde{Z}=\widetilde{E}=W_{0}(\mathbb{R}) U_{0}(\mathbb{C}) \widetilde{z}
$$

for some point $\widetilde{z} \in \widetilde{Z}_{v}$.

Step $\boldsymbol{I} \boldsymbol{V}$. Let us now conclude that $\widetilde{Z}$ is weakly special.

First of all, $U_{0} \triangleleft P$ by Proposition 2.6(2). Consider $\left(P, \mathcal{X}^{+}\right) \stackrel{\rho}{\rightarrow}\left(P, \mathcal{X}^{+}\right) / U_{0}$, then by definition $\widetilde{Z}$ is weakly special iff $\rho(\widetilde{Z})$ is. Replace $\left(P, \mathcal{X}^{+}\right)\left(\operatorname{resp} . W, \widetilde{Z}, W_{0}, \widetilde{z}\right)$ by $\left(P, \mathcal{X}^{+}\right) / U_{0}$ (resp. $\left.W / U_{0}, \rho(\widetilde{Z}), W_{0} / U_{0}=V_{0}, \rho(\widetilde{z})\right)$, then $V_{0}$ is a subgroup of $W$ and $\widetilde{Z}=V_{0}(\mathbb{R}) \widetilde{z}$. Use the notation of $\S 2.2$ and $\S 4$ and suppose $\widetilde{z}=\left(\widetilde{z}_{U}, \widetilde{z}_{V}\right)$. By Proposition 7.2, $\widetilde{Z}$ is weakly special iff $\widetilde{z}_{V} \in\left(N_{W}\left(V_{0}\right) / U\right)(\mathbb{R})$ iff $\Psi\left(V_{0}(\mathbb{R}), \widetilde{z}_{V}\right)=0$. We shall prove the last claim.

Define $Z:=\operatorname{unif}(\widetilde{Z}), z=\operatorname{unif}(\widetilde{z})$ and $z_{V}=[\pi](z) \in A$, then $\pi(\widetilde{Z})=V_{0}(\mathbb{R})+\widetilde{z}_{V}$ and $[\pi](Z)=A_{0}+z_{V}$ where $A_{0}=\Gamma_{V_{0}} \backslash V_{0}(\mathbb{R})$ is an abelian subvariety of $A$. We can compute the fiber

$$
Z_{z_{V}}=\left(\operatorname{unif}\left(\Gamma_{W} \widetilde{Z}\right)\right)_{z_{V}}=\widetilde{z}_{U}+\frac{1}{2} \Psi\left(\Gamma_{V}, \widetilde{z}_{V}\right)+\Gamma_{U} \bmod \Gamma_{U}
$$

We have $\Psi(V(\mathbb{R}), V(\mathbb{R})) \subset U(\mathbb{R})$ since $\Psi$ is defined over $\mathbb{Q}$. Let us prove $\Psi\left(\Gamma_{V}, \widetilde{z}_{V}\right) \subset U(\mathbb{Q})$. Fix an isomorphism $\Gamma_{U} \simeq \mathbb{Z}^{m}$, which induces an isomorphism $U(\mathbb{Q}) \simeq \mathbb{Q}^{m}$. Suppose that there exists a $u \in \Psi\left(\Gamma_{V}, \widetilde{z}_{V}\right) \backslash U(\mathbb{Q})$, then at least one of the coordinates of $u$ is irrational. Without loss 
of generality we may assume that its first coordinate $u_{1} \in \mathbb{R} \backslash \mathbb{Q}$. Denote by $U_{1}$ the $\mathbb{Q}$-subgroup of $U$ corresponding to the first factor of $U(\mathbb{Q}) \simeq \mathbb{Q}^{m}$, then

$$
\operatorname{unif}\left(\widetilde{z}_{U}+U_{1}(\mathbb{R})\right) \subset \overline{Z_{z_{V}}}
$$

since $\left\{l u_{1} \bmod \mathbb{Z} \mid l \in \mathbb{Z}\right\}$ is dense in $[0,1)$. So $\overline{Z_{z_{V}}}$ contains

$$
\operatorname{unif}\left(\widetilde{z}_{U}+U_{1}(\mathbb{C})\right) \text {, }
$$

and so does $Y_{z_{V}}$ since $\bar{Z} \subset Y$. Let $v:=v_{0}+\widetilde{z}_{V} \in V(\mathbb{R})$, then $\widetilde{z}_{U}+U_{1}(\mathbb{C}) \subset$ unif $^{-1}(Y)_{v}$. However $\widetilde{Z}_{\widetilde{z}_{v}}=\widetilde{z}_{U}$ by (11.7) (recall that we have reduced to $W_{0}=V_{0}$ and $U_{0}=0$ ), which contradicts (11.6). Hence $\Psi\left(\Gamma_{V}, \widetilde{z}_{V}\right) \subset U(\mathbb{Q})$, and therefore $(1 / 2) \Psi\left(N \Gamma_{V}, \widetilde{z}_{V}\right) \subset \Gamma_{U}$ for some $N \gg 0$ (since $\left.\operatorname{rank} \Gamma_{V}<\infty\right)$. Now we can construct a new lattice $\Gamma_{W}^{\prime}$ with $N \Gamma_{V}$ and $\Gamma_{U}$. $\Gamma_{W}^{\prime}$ is of finite index in $\Gamma_{W}$. Replacing $\Gamma_{W}$ by $\Gamma_{W}^{\prime}$ does not change the assumption or the conclusion of Ax-Lindemann-Weierstraß, so we may assume $(1 / 2) \Psi\left(\Gamma_{V}, \widetilde{z}_{V}\right) \subset \Gamma_{U}$. Now we can define $C^{\infty}$-morphisms

$$
\begin{aligned}
f: & A_{0}+z_{V} \\
a_{0}+z_{V} & \mapsto \widetilde{z}_{U}+(1 / 2) \Psi\left(v_{0}, \widetilde{z}_{V}\right) \bmod \Gamma_{U}
\end{aligned}
$$

and

$$
\begin{aligned}
s: & A_{0}+z_{V} \\
& a_{0}+z_{V} \mapsto\left(\widetilde{z}_{U}+(1 / 2) \Psi\left(v_{0}, \widetilde{z}_{V}\right), a_{0}+z_{V}\right) \bmod \Gamma_{W}
\end{aligned}
$$

where $v_{0}$ is any point of $V_{0}(\mathbb{R})$ such that $\operatorname{unif}_{V}\left(v_{0}\right)=a_{0}$. But $Z_{a}$ is a single point for all $a \in A_{0}+z_{V}$ by (11.8), so $s$ is the inverse of $\left.[\pi]\right|_{Z}$, and therefore $s$ is a holomorphic section of $\left.E\right|_{A_{0}+z_{V}} \rightarrow A_{0}+z_{V}$. Locally on $U_{i} \subset A_{0}+z_{V}, s$ is represented by a holomorphic morphism $U_{i} \rightarrow T$, which must equal to $\left.f\right|_{U_{i}}$ by definition. Hence $f$ is holomorphic since being holomorphic is a local condition. So $f$ is constant.

But $\Psi\left(0, \widetilde{z}_{V}\right)=0$, and therefore $(1 / 2) \Psi\left(V_{0}(\mathbb{R}), \widetilde{z}_{V}\right) \subset \Gamma_{U}$. But $\Psi\left(V_{0}(\mathbb{R}), \widetilde{z}_{V}\right)$ is continuous and $\Psi\left(0, \widetilde{z}_{V}\right)=0$, so $\Psi\left(V_{0}(\mathbb{R}), \widetilde{z}_{V}\right)=0$. Hence we are done.

\section{Consequence of Ax-Lindemann-Weierstrass}

12.1. Weakly special subvarieties defined by a fixed $\mathbb{Q}$-subgroup. Let $S=\Gamma \backslash \mathcal{X}^{+}$be a connected mixed Shimura variety associated with the connected mixed Shimura datum $\left(P, \mathcal{X}^{+}\right)$ and let unif: $\mathcal{X}^{+} \rightarrow S$ be the uniformization. Suppose that $N$ is a connected subgroup of $P$ s.t. $N /(W \cap N) \hookrightarrow G$ is semi-simple. A subvariety of $S$ is said to be weakly special defined by $N$ if it is of the form unif $\left(i\left(\varphi^{-1}\left(y^{\prime}\right)\right)\right)$ under the notation of Definition 5.2 s.t. $N=\operatorname{Ker}(\varphi)$. Let $\mathfrak{F}(N)$ be the set of all weakly special subvarieties of $S$ defined by $N$. The goal of this subsection is to prove:

Proposition 12.1. If $\mathfrak{F}(N) \neq \emptyset$ and $N \Varangle P$, then $\cup_{Z \in \mathfrak{F}(N)} Z$ is a finite union of proper special subvarieties of $S$.

Proof. Take any $F \in \mathfrak{F}(N)$. Let $\mathcal{F}$ be a fundamental domain for the action $\Gamma$ on $\mathcal{X}^{+}$. Suppose that $x^{\prime} \in \mathcal{F}$ is such that $F=\operatorname{unif}\left(N(\mathbb{R})^{+} U_{N}(\mathbb{C}) x^{\prime}\right)$. Consider $Q^{\prime}:=N_{P}(N)$, the normalizer of $N$ in $P$. By definition of weakly special subvarieties, there exists $\left(R^{\prime}, \mathcal{Z}^{+}\right) \hookrightarrow\left(P, \mathcal{X}^{+}\right)$such that $h_{x^{\prime}}: \mathbb{S}_{\mathbb{C}} \rightarrow P_{\mathbb{C}}$ factors through $R_{\mathbb{C}}^{\prime}$ and $N \triangleleft R^{\prime}$. Hence $R^{\prime}<Q^{\prime}$. Define $G_{Q^{\prime}}:=Q^{\prime} /\left(W \cap Q^{\prime}\right)$. 
Then $G_{Q^{\prime}} /\left(Z(G) \cap G_{Q^{\prime}}\right)$ is reductive by [17, Lemma 4.3] or [60, Proposition 3.28], and hence $G_{Q^{\prime}}$ is reductive. Write

$$
1 \rightarrow W \cap Q^{\prime} \rightarrow Q^{\prime} \stackrel{\pi_{Q^{\prime}}}{\longrightarrow} G_{Q^{\prime}} \rightarrow 1
$$

The group $G_{Q^{\prime}}=Z\left(G_{Q^{\prime}}\right)^{\circ} G_{Q^{\prime}}^{\mathrm{nc}} G_{Q^{\prime}}^{\mathrm{c}}$ is an almost-direct product, where $G_{Q^{\prime}}^{\mathrm{nc}}$ (resp. $G_{Q^{\prime}}^{\text {c }}$ ) is the product of the $\mathbb{Q}$-simple factors whose set of $\mathbb{R}$-points is non-compact (resp. compact). Let $G_{Q}:=Z\left(G_{Q^{\prime}}\right)^{\circ} G_{Q^{\prime}}^{\text {nc }}$ and then define $Q:=\pi_{Q^{\prime}}^{-1}\left(G_{Q}\right)$, then $h_{x^{\prime}}$ factors through $Q_{\mathbb{C}}$ and $R^{\prime}<Q$ by Definition 2.1(4). So $N \triangleleft Q$ and $\left(Q, \mathcal{Y}^{+}\right)$, where $\mathcal{Y}^{+}:=Q(\mathbb{R})^{+} U_{Q}(\mathbb{C}) x^{\prime}$, is a connected mixed Shimura subdatum of $\left(P, \mathcal{X}^{+}\right)$. But then $F \subset \operatorname{unif}\left(\mathcal{Y}^{+}\right) \subset \cup_{Z \in \mathfrak{F}(N)} Z$.

Define $\mathfrak{Y}_{Q}:=\left\{x \in \mathcal{X}^{+} \mid h_{x}\right.$ factors through $\left.Q_{\mathbb{C}}\right\}$, then $Q(\mathbb{R})^{+} U_{Q}(\mathbb{C}) \mathfrak{Y}_{Q}=\mathfrak{Y}_{Q}$. The discussion of last paragraph tells us that $F \subset \operatorname{unif}\left(\mathfrak{Y}_{Q}\right)$ for any $F \in \mathfrak{F}(N)$. On the other hand, for any $x \in \mathfrak{Y}_{Q},\left(Q, \mathcal{Y}^{+}\right)$, where $\mathcal{Y}^{+}:=Q(\mathbb{R})^{+} U_{Q}(\mathbb{C}) x$, is a connected mixed Shimura subdatum of $\left(P, \mathcal{X}^{+}\right)$and hence $\operatorname{unif}\left(N(\mathbb{R})^{+} U_{N}(\mathbb{C}) x\right) \in \mathfrak{F}(N)$. Therefore unif $\left(\mathfrak{Y}_{Q}\right) \subset \cup_{Z \in \mathfrak{F}(N)} Z$. To sum it up, $\cup_{Z \in \mathfrak{F}(N)} Z=\operatorname{unif}\left(\mathfrak{Y}_{Q}\right)$.

Now we are done if we can prove

Claim. The set $\mathfrak{Y}_{Q}$ is a finite union of $Q(\mathbb{R})^{+} U_{Q}(\mathbb{C})$-conjugacy classes. In other words, $\mathfrak{Y}_{Q}$ is a finite union of connected mixed Shimura subdata of $\left(P, \mathcal{X}^{+}\right)$.

Fix a special point $x$ of $\mathcal{X}^{+}$contained in $\mathfrak{Y}_{Q}$. There exists by definition a torus $T_{x} \subset Q$ such that $h_{x}: \mathbb{S}_{\mathbb{C}} \rightarrow Q_{\mathbb{C}}$ factors through $T_{x, \mathbb{C}}$. Furthermore, we may and do assume that $T_{x, \mathbb{C}}$ is a maximal torus of $Q_{\mathbb{C}}$. Let $T$ be a maximal torus of $P_{\mathbb{C}}$ defined over $\mathbb{Q}$ such that $T>T_{x}$. Take a Levi decomposition $P=W \rtimes G$ such that $T<G<P$. Then the composite $\mathbb{S}_{\mathbb{C}} \stackrel{h_{x}}{\rightarrow} T_{x, \mathbb{C}}<P_{\mathbb{C}} \stackrel{\pi}{\rightarrow} G_{\mathbb{C}}<P_{\mathbb{C}}$ equals $h_{x}$ and is defined over $\mathbb{R}$ by Definition 2.1(1).

For any other special point $y$ of $\mathcal{X}^{+}$contained in $\mathfrak{Y}_{Q}$, there exists $g \in Q(\mathbb{C})$ such that $g T_{x, \mathbb{C}} g^{-1}=T_{y, \mathbb{C}}$. The number of the $Q(\mathbb{R})$-conjugacy classes of maximal tori of $Q_{\mathbb{R}}$ defined over $\mathbb{R}$ is at most

$$
\#\left(\operatorname{Ker}\left(H^{1}\left(\mathbb{R}, N_{Q(\mathbb{R})}\left(T_{x, \mathbb{R}}\right)\right) \rightarrow H^{1}(\mathbb{R}, Q)\right)\right)<\infty,
$$

where $N_{Q(\mathbb{R})}\left(T_{x, \mathbb{R}}\right)$ is the normalizer of $T_{x, \mathbb{R}}$ in $Q(\mathbb{R})$. So it is equivalent to prove the finiteness of the $Q(\mathbb{R})^{+} U_{Q}(\mathbb{C})$-conjugay classes in $\mathfrak{Y}_{Q}$ and to prove the finiteness of the $Q(\mathbb{R})^{+}$-conjugacy classes of the morphisms $\mathbb{S} \rightarrow T_{x, \mathbb{R}}$. But $T_{x}<T<G$, so the $Q(\mathbb{R})^{+}$-conjugacy classes of the morphisms $\mathbb{S} \rightarrow T_{x, \mathbb{R}}$ equals the $G_{Q}(\mathbb{R})^{+}$-conjugacy classes of the morphisms $\mathbb{S} \rightarrow T_{x, \mathbb{R}}$. In otherwords, it suffices to prove the claim for $\left(G, \mathcal{X}_{G}^{+}\right)$. Now the result follows from [17, Lemma 4.4(ii)] (or [40, 2.4] or [63, Lemma 3.7]).

12.2. Consequence of Ax-Lindemann-Weierstraß. Now we use the result of the previous subsection to prove the following theorem, which will be used in the next section to prove the André-Oort Conjecture.

Theorem 12.2. Let $S=\Gamma \backslash \mathcal{X}^{+}$be a connected mixed Shimura variety associated with the connected mixed Shimura datum $\left(P, \mathcal{X}^{+}\right)$. Let $Y$ be a Hodge generic irreducible subvariety of $S$. Then there exists an $N \triangleleft P$ (denote by $\left.U_{N}:=U \cap N\right)$ s.t. for the diagram

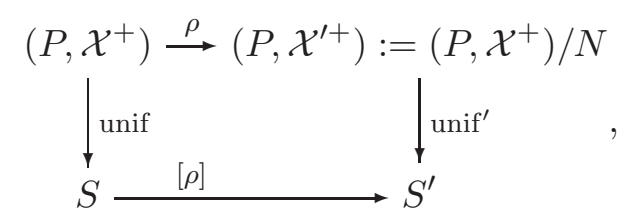


- the union of positive-dimensional weakly special subvarieties which are contained in $Y^{\prime}:=$ $\overline{[\rho](Y)}$ is NOT Zariski dense in $Y^{\prime}$;

- $Y=[\rho]^{-1}\left(Y^{\prime}\right)$.

Proof. Without any loss of generality, we assume that the union of positive-dimensional weakly special subvarieties which are contained in $Y$ is Zariski dense in $Y$.

Take a fundamental domain $\mathcal{F}$ for the action of $\Gamma$ on $\mathcal{X}^{+}$s.t. unif $\left.\right|_{\mathcal{F}}$ is definable. Such an $\mathcal{F}$ exists by e.g. $§ 10.1$.

By Reduction Lemma (Lemma 2.12), we may assume $\left(P, \mathcal{X}^{+}\right) \stackrel{\lambda}{\longrightarrow}\left(G_{0}, \mathcal{D}^{+}\right) \times \prod_{i=1}^{r}\left(P_{2 g}, \mathcal{X}_{2 g}^{+}\right)$, i.e. replace $\left(P, \mathcal{X}^{+}\right)$by $\left(P^{\prime}, \mathcal{X}^{\prime+}\right)$ in the lemma if necessary. Identify $\left(P, \mathcal{X}^{+}\right)$with its image under $\lambda$.

Let $\mathcal{T}$ be the set of the triples $\left(U^{\prime}, V^{\prime}, G^{\prime}\right)$ consisting of an $\mathbb{R}$-subgroup of $U_{\mathbb{R}}$, an $\mathbb{R}$-sub-Hodge structure of $V_{\mathbb{R}}$ and a connected $\mathbb{R}$-subgroup of $G_{\mathbb{R}}$ which is semi-simple and has no compact factors. Let

$$
\mathcal{G}:=\mathbb{G}_{m}(\mathbb{R})^{r} \times \mathrm{GSp}_{2 g}(\mathbb{R}) \times G(\mathbb{R}),
$$

then $\mathcal{G}$ acts on $\mathcal{T}$ by $\left(g_{U}, g_{V}, g\right) \cdot\left(U^{\prime}, V^{\prime}, G^{\prime}\right):=\left(g_{U} U^{\prime}, g_{V} V^{\prime}, g G^{\prime} g^{-1}\right)$. Also we define the action of a triple $\left(U^{\prime}(\mathbb{R}), V^{\prime}(\mathbb{R}), G^{\prime}(\mathbb{R})\right)$ on $\mathcal{X}^{+} \simeq U(\mathbb{C}) \times V(\mathbb{R}) \times \mathcal{X}_{G}^{+}$as $(4.2)$. This action is algebraic.

Lemma 12.3. Up to the action of $\mathcal{G}$ on $\mathcal{T}$, there exist only finitely many such triples.

Proof. First of all by root system and Galois cohomology, there exist only finitely many semisimple subgroups of $G_{\mathbb{R}}$ up to conjugation by $G(\mathbb{R})$.

Secondly, $V^{\prime}$ is by definition a symplectic subspace of $V_{\mathbb{R}}$. Hence a symplectic base of $V^{\prime}$ extends to a symplectic base of $V_{\mathbb{R}}=V_{2 g, \mathbb{R}}$. But $\mathrm{GSp}_{2 g}(\mathbb{R})$ acts transitively on the set of symplectic bases of $V_{2 g, \mathbb{R}}$, so there are only finitely many choices for $V^{\prime}$ up to the action of $\mathrm{GSp}_{2 g}(\mathbb{R})$ (in fact, there are only $g$ choices).

Finally, observe that $\forall\left(\lambda_{1}, \ldots, \lambda_{r}\right) \in \mathbb{G}_{m}(\mathbb{R})^{r}$ and $\left(u_{1}, \ldots, u_{r}\right) \in U \simeq \oplus_{i=1}^{r} U_{2 g}^{(i)}$,

$$
\left(\lambda_{1}, \ldots, \lambda_{r}\right) \cdot\left(u_{1}, \ldots, u_{r}\right)=\left(\lambda_{1} u_{1}, \ldots, \lambda_{r} u_{r}\right)
$$

Now it is clear that $\left(u_{1}, \ldots, u_{r}\right)$ and $\left(u_{1}^{\prime}, \ldots, u_{r}^{\prime}\right)$ are under the same orbit of the action of $\mathbb{G}_{m}(\mathbb{R})^{r}$ if and only if $u_{i} u_{i}^{\prime} \geqslant 0$ with $u_{i} u_{i}^{\prime}=0 \Rightarrow u_{i}=u_{i}^{\prime}=0$ for all $i=1, \ldots, r$. Hence up to the action of $\mathbb{G}_{m}(\mathbb{R})^{r}$, there are only finitely many $U^{\prime \prime}$ s (in fact, there are $2\left(\begin{array}{l}r \\ s\end{array}\right) U^{\prime \prime}$ s of dimension $s$ ).

Let $\mathfrak{W}(Y)$ (resp. $\mathfrak{W}_{l}(Y)$ ) be the union of weakly special subvarieties of positive dimension (resp. of real dimension $l$ ) contained in $Y$.

For any $l$ s.t. $\mathfrak{W}_{l}(Y) \neq \emptyset$, there exist by definition (and Proposition 5.4) a subgroup $N_{l}$ of $P^{\text {der }}$ and a point $x_{0} \in \mathcal{F}$ s.t. $\operatorname{unif}\left(N_{l}(\mathbb{R})^{+} U_{N_{l}}(\mathbb{C}) x_{0}\right)$ is a weakly special subvariety of dimension $l$ contained in $Y$. Note that the triple $\left(U_{N_{l}, \mathbb{R}}, V_{N_{l}, \mathbb{R}}, G_{N_{l}, \mathbb{R}}^{+ \text {nc }}\right) \in \mathcal{T}$, where $G_{N_{l}, \mathbb{R}}^{+ \text {nc }}$ is the product of the $\mathbb{R}$-simple factors of $G_{N_{l}, \mathbb{R}}^{+}$which are non-compact. We say that two such subgroups $N_{l}, N_{l}^{\prime}$ of $P$ are equivalent if $\left(U_{N_{l}, \mathbb{R}}, V_{N_{l}, \mathbb{R}}, G_{N_{l}, \mathbb{R}}^{+\mathrm{nc}}\right)=\left(U_{N_{l}^{\prime}, \mathbb{R}}, V_{N_{l}^{\prime}, \mathbb{R}}, G_{N_{l}^{\prime}, \mathbb{R}}^{+\mathrm{nc}}\right)$. By condition (4) of Definition 2.1, unif $\left(N_{l}(\mathbb{R})^{+} U_{N_{l}}(\mathbb{C}) x_{0}\right)=\operatorname{unif}\left(N_{l}^{\prime}(\mathbb{R})^{+} U_{N_{l}^{\prime}}(\mathbb{C}) x_{0}\right)$ iff $N_{l}$ and $N_{l}^{\prime}$ are equivalent.

Define

$$
B\left(N_{l, \mathbb{R}}, Y\right):=\left\{\left(g_{U}, g_{V}, g, x\right) \in \mathcal{G} \times \mathcal{F} \mid \operatorname{unif}\left(\left(g_{U} U_{N_{l}}(\mathbb{C}), g_{V} V_{N_{l}}(\mathbb{R}), g G_{N_{l}}(\mathbb{R})^{+\mathrm{nc}} g^{-1}\right) x\right) \subset Y\right.
$$
and is not contained in $\left.\cup_{l^{\prime}>l} \mathfrak{W}_{l^{\prime}}(Y)\right\}$. 
Then by analytic continuation, (12.2)

$$
\begin{array}{r}
B\left(N_{l, \mathbb{R}}, Y\right)=\left\{\left(g_{U}, g_{V}, g, x\right) \in \mathcal{G} \times \mathcal{F}|p r|_{\mathcal{F}}\left(\left(g_{U} U_{N_{l}}(\mathbb{R}), g_{V} V_{N_{l}}(\mathbb{R}), g G_{N_{l}}(\mathbb{R})^{+\mathrm{nc}} g^{-1}\right) x\right) \subset Y\right. \\
\text { and is not contained in } \cup_{l^{\prime}}>l \\
\left.\mathfrak{W}_{l^{\prime}}(Y)\right\} .
\end{array}
$$

Lemma 12.4. For any $\left(g_{U}, g_{V}, g, x\right) \in B\left(N_{l, \mathbb{R}}, Y\right)$, define

$$
\widetilde{Z}:=\left(g_{U} U_{N_{l}}(\mathbb{C}), g_{V} V_{N_{l}}(\mathbb{R}), g G_{N_{l}}(\mathbb{R})^{+\mathrm{nc}} g^{-1}\right) x .
$$

Then unif $(\widetilde{Z})$ is a weakly special subvariety of $Y$.

Proof. The set $\widetilde{Z}$ is a connected irreducible semi-algebraic subset of $\mathcal{X}^{+}$which is contained in unif $^{-1}(Y)$. Let $\widetilde{Z}^{\dagger}$ be a connected irreducible semi-algebraic subset of $\mathcal{X}^{+}$which is contained in unif $^{-1}(Y)$ and which contains $\widetilde{Z}$, maximal for these properties. By [50, Lemma 4.1] (there is a typo in the proof: $\mathbb{C}^{2 n}$ should be $\mathbb{C}^{n}$ ) and Ax-Lindemann-Weierstraß (Theorem 1.2), $\widetilde{Z}^{\dagger}$ is complex analytic and each of its complex analytic irreducible component is weakly special. But $\widetilde{Z}$ is smooth, so $\widetilde{Z}$ is contained in one complex analytic irreducible component of $\widetilde{Z}^{\dagger}$ which we denote by $\widetilde{Z}^{\prime}$. Now we have

$$
\begin{aligned}
\operatorname{dim}(\widetilde{Z})-\operatorname{dim}\left(N_{l}(\mathbb{R})^{+} U_{N_{l}}(\mathbb{C}) x_{0}\right) & =\operatorname{dim}\left(g G_{N_{l}}(\mathbb{R})^{+} g^{-1} \cdot x_{G}\right)-\operatorname{dim}\left(G_{N_{l}}(\mathbb{R})^{+} x_{0, G}\right) \\
& =\operatorname{dim}\left(\operatorname{Stab}_{G_{N_{l}}(\mathbb{R})^{+}}\left(x_{0, G}\right)\right)-\operatorname{dim}\left(\operatorname{Stab}_{\left.g G_{N_{l}}(\mathbb{R})^{+} g^{-1}\left(x_{G}\right)\right)}\right. \\
& \geqslant 0
\end{aligned}
$$

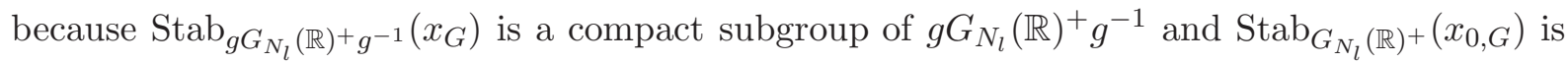
a maximal compact subgroup of $G_{N_{l}}(\mathbb{R})^{+}$. Hence

$$
\operatorname{dim}\left(\widetilde{Z}^{\prime}\right) \leqslant l=\operatorname{dim}\left(N_{l}(\mathbb{R})^{+} U_{N_{l}}(\mathbb{C}) x_{0}\right) \leqslant \operatorname{dim}(\widetilde{Z}) \leqslant \operatorname{dim}\left(\widetilde{Z}^{\prime}\right)
$$

where the first inequality follows from the definition of $B\left(N_{l, \mathbb{R}}, Y\right)$. Therefore $\widetilde{Z}=\widetilde{Z}^{\prime}$ is weakly special. So $\operatorname{unif}(\widetilde{Z})$ is weakly special.

Define

$$
\begin{array}{r}
C\left(N_{l, \mathbb{R}}, Y\right):=\left\{\underline{t}:=\left(g_{U} U_{N_{l}}(\mathbb{R}), g_{V} V_{N_{l}}(\mathbb{R}), g G_{N_{l}}(\mathbb{R})^{+ \text {nc }} g^{-1}\right) \mid\left(g_{U}, g_{V}, g\right) \in \mathcal{G} \text { s.t. } \exists x \in \mathcal{F}\right. \\
\text { with unif } \left.(\underline{t} \cdot x) \subset Y \text { and is not contained in } \cup_{l^{\prime}>l} \mathfrak{W}_{l^{\prime}}(Y)\right\}
\end{array} .
$$

Let

$$
\begin{gathered}
B\left(N_{l, \mathbb{R}}, Y\right) \stackrel{\psi_{l}}{\longrightarrow}\left(\mathbb{G}_{m}(\mathbb{R})^{r} / \operatorname{Stab}_{\mathbb{G}_{m}(\mathbb{R})^{r}} U_{N_{l}}(\mathbb{R})\right) \times \mathrm{GSp}_{2 g}(\mathbb{R}) / \operatorname{Stab}_{\mathrm{GSp}_{2}(\mathbb{R})} V_{N_{l}}(\mathbb{R}) \times G(\mathbb{R}) / N_{G(\mathbb{R})} G_{N_{l}}(\mathbb{R})^{+\mathrm{nc}} \\
\left(g_{U}, g_{V}, g, x\right) \mapsto c
\end{gathered},
$$

then there is a bijection between $\psi_{l}\left(B\left(N_{l, \mathbb{R}}, Y\right)\right)$ and $C\left(N_{l, \mathbb{R}}, Y\right)$.

Lemma 12.5. The set $C\left(N_{l, \mathbb{R}}, Y\right)$ (hence $\left.\psi_{l}\left(B\left(N_{l, \mathbb{R}}, Y\right)\right)\right)$ is countable.

Proof. By Lemma 12.4, unif $\left(\left(g_{U} U_{N_{l}}(\mathbb{C}), g_{V} V_{N_{l}}(\mathbb{R}), g G_{N_{l}}(\mathbb{R})^{+\mathrm{nc}} g^{-1}\right) \cdot x\right)$ is weakly special. Hence by Proposition 5.4 there exists a $\mathbb{Q}$-subgroup $N^{\prime}$ of $P^{\text {der }}$ s.t.

$$
\left(g_{U} U_{N_{l}}(\mathbb{C}), g_{V} V_{N_{l}}(\mathbb{R}), g G_{N_{l}}(\mathbb{R})^{+\mathrm{nc}} g^{-1}\right)=\left(U_{N^{\prime}}(\mathbb{C}), V_{N^{\prime}}(\mathbb{R}), G_{N^{\prime}}(\mathbb{R})^{+\mathrm{nc}}\right) .
$$

But $g_{U} U_{N_{l}}(\mathbb{R})=g_{U} U_{N_{l}}(\mathbb{C}) \cap U(\mathbb{R})$ and $U_{N^{\prime}}(\mathbb{R})=U_{N^{\prime}}(\mathbb{C}) \cap U(\mathbb{R})$, so

$$
\left(g_{U} U_{N_{l}}(\mathbb{R}), g_{V} V_{N_{l}}(\mathbb{R}), g G_{N_{l}}(\mathbb{R})^{+\mathrm{nc}} g^{-1}\right)=\left(U_{N^{\prime}}(\mathbb{R}), V_{N^{\prime}}(\mathbb{R}), G_{N^{\prime}}(\mathbb{R})^{+\mathrm{nc}}\right) .
$$


So $C\left(N_{l, \mathbb{R}}, Y\right)$, and therefore $\psi_{l}\left(B\left(N_{l, \mathbb{R}}, Y\right)\right)$ is countable.

Proposition 12.6. For any $l>0$ and $N_{l}$,

(1) the set $C\left(N_{l, \mathbb{R}}, Y\right)$ (hence $\left.\psi_{l}\left(B\left(N_{l, \mathbb{R}}, Y\right)\right)\right)$ is finite;

(2) the set $\cup_{l^{\prime} \geqslant l} \mathfrak{W}_{l^{\prime}}(Y)$ is definable;

Proof. We prove the two statements together by induction on $l$.

Step I. Let $d$ be the maximum of the dimensions of weakly special subvarieties of positive dimension contained in $Y$. For any $N_{d}, B\left(N_{d, \mathbb{R}}, Y\right)$ is definable by (12.2), and hence $\psi_{d}\left(B\left(N_{d, \mathbb{R}}, Y\right)\right)$ is definable since $\psi_{d}$ is algebraic. So $\psi_{d}\left(B\left(N_{d, \mathbb{R}}, Y\right)\right)$, and therefore $C\left(N_{d, \mathbb{R}}, Y\right)$, is finite by Lemma 12.5 .

Consider all the triples

$$
\mathfrak{W}_{d}(Y, \mathcal{T}):=\left\{\left(U^{\prime}, V^{\prime}, G^{\prime}\right) \in \mathcal{T} \mid \begin{array}{r}
\exists x \in \mathcal{F} \text { with unif }\left(\left(U^{\prime}(\mathbb{C}), V^{\prime}(\mathbb{R}), G^{\prime}(\mathbb{R})^{+}\right) x\right) \\
\text { weakly special of dimension } d \text { contained in } Y\} .
\end{array}\right.
$$

By Lemma 12.3, any $\underline{t} \in \mathfrak{W}_{d}(Y, \mathcal{T})$ is of the form $\underline{g} \cdot\left(U_{i}^{\prime}, V_{i}^{\prime}, G_{i}^{\prime}\right)$ for $\underline{g} \in \mathcal{G}$ and $i=1, \ldots, n$. Furthermore, by Proposition 5.4, we may assume

$$
\left(U_{i}^{\prime}, V_{i}^{\prime}, G_{i}^{\prime}\right)=\left(U_{N_{i}^{\prime}, \mathbb{R}}, V_{N_{i}^{\prime}, \mathbb{R}}, G_{N_{i}^{\prime}, \mathbb{R}}^{+\mathrm{nc}}\right)
$$

for some $N_{i}^{\prime}<Q(i=1, \ldots, n)$. But we just proved that $C\left(N_{i, \mathbb{R}}^{\prime}, Y\right)$ is finite $(\forall i=1, \ldots, n)$. Hence $\mathfrak{W}_{d}(Y, \mathcal{T})$ is a finite set. Again by Propostition 5.4, each triple of $\mathfrak{W}_{d}(Y, \mathcal{T})$ equals $\left(U_{N^{\prime}, \mathbb{R}}, V_{N^{\prime}, \mathbb{R}}, G_{N^{\prime}, \mathbb{R}}^{+\mathrm{nc}}\right)$ for some $N^{\prime}<P$. We shall denote this triple by $N^{\prime}$ for simplicity.

Hence

$$
\mathfrak{W}_{d}(Y)=\bigcup_{N^{\prime} \in \mathfrak{W}_{d}(Y, \mathcal{T})} \bigcup_{\substack{(0,0,1, x) \\ \in B\left(N_{\mathbb{R}}^{\prime}, Y\right)}} \operatorname{unif}\left(\left(N^{\prime}(\mathbb{R})^{+} U_{N^{\prime}}(\mathbb{C}) x\right)\right.
$$

is definable.

Step II. For any $l$ and $N_{l}, B\left(N_{l, \mathbb{R}}, Y\right)$ is definable by (12.2) and induction hypothesis (2). Arguing as in the previous case we get that $C\left(N_{l, \mathbb{R}}, Y\right)$ is finite. Define

$$
\mathfrak{W}_{l}(Y, \mathcal{T}):=\left\{\left(U^{\prime}, V^{\prime}, G^{\prime}\right) \in \mathcal{T} \mid \begin{array}{r}
\exists x \in \mathcal{F} \text { with unif }\left(\left(U^{\prime}(\mathbb{C}), V^{\prime}(\mathbb{R}), G^{\prime}(\mathbb{R})^{+}\right) x\right) \text { weakly special } \\
\text { of dimension } \left.l \text { contained in } Y \text { but not contained in } \cup_{l^{\prime}>l} \mathfrak{W}_{l^{\prime}}(Y)\right\} .
\end{array}\right.
$$

Arguing as in the previous case we can get that $\mathfrak{W}_{l}(Y, \mathcal{T})$ is a finite set and each element of it equals $\left(U_{N^{\prime}, \mathbb{R}}, V_{N^{\prime}, \mathbb{R}}, G_{N^{\prime}, \mathbb{R}}^{+ \text {nc }}\right)$ for some $N^{\prime}<P$. Hence

$$
\bigcup_{l^{\prime} \geqslant l} \mathfrak{W}_{l^{\prime}}(Y)=\bigcup_{l^{\prime}>l} \mathfrak{W}_{l^{\prime}}(Y) \cup \bigcup_{N^{\prime} \in \mathfrak{W}_{l}(Y, \mathcal{T})} \bigcup_{\substack{(0,0,1, x) \\ \in B\left(N_{\mathbb{R}}^{\prime}, Y\right)}} \operatorname{unif}\left(N^{\prime}(\mathbb{R})^{+} U_{N^{\prime}}(\mathbb{C}) x\right)
$$

is definable by induction hypothesis (2).

From now on, for any connected subgroup $N^{\dagger}$ of $P$, we will denote by $\mathfrak{F}\left(N^{\dagger}\right)$ the set of all weakly special subvarieties of $S$ defined by the group $N^{\dagger}$ (see the beginning of this section) and $\mathfrak{F}\left(N^{\dagger}, Y\right):=\left\{Z \in \mathfrak{F}\left(N^{\dagger}\right)\right.$ s.t. $\left.Z \subset Y\right\}$. Remark that when proving Proposition 12.6, we have also given the following description of $\mathfrak{W}(Y)=\cup_{l=1}^{d} \mathfrak{W}_{l}(Y)$ :

$$
\mathfrak{W}(Y)=\bigcup_{N^{\prime}} \operatorname{unif}\left(N^{\prime}(\mathbb{R})^{+} U_{N}^{\prime}(\mathbb{C}) \text {-orbits contained in unif }{ }^{-1}(Y)\right)=\bigcup_{N^{\prime}} \bigcup_{Z \in \mathfrak{F}\left(N^{\prime}, Y\right)} Z
$$


which is a finite union on $N^{\prime}$ 's and each $N^{\prime}$ is of positive dimension. We have assumed that $\mathfrak{W}(Y)$ is Zariski dense in $Y$ (otherwise there is nothing to prove). Therefore by (12.4), there exists an $N_{1}$ of positive dimension s.t.

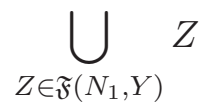

is Zariski dense in $Y$.

Prove now $N_{1} \triangleleft P$. If not, then by Proposition 12.1, $\cup_{Z \in \mathfrak{F}\left(N_{1}\right)} Z$ equals a finite union of proper special subvarieties of $S$. The intersection of this union and $Y$ is not Zariski dense in $Y$ since $Y$ is Hodge generic in $S$. This is a contradiction. Hence $N_{1} \triangleleft P$.

Consider the diagram

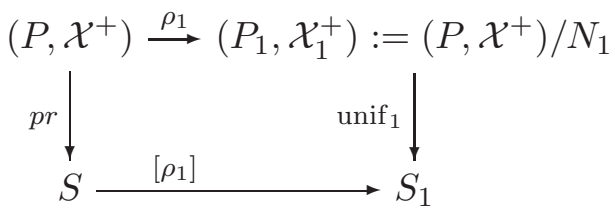

and let $Y_{1}:=\overline{\left[\rho_{1}\right](Y)}$, which is Hodge generic in $S_{1}$. Since $\operatorname{dim}\left(N_{1}\right)>0, \operatorname{dim}\left(S_{1}\right)<\operatorname{dim}(S)$. It is not hard to prove $[\rho]^{-1}\left(Y_{1}\right)=Y$ by the fact (12.5). If the union of positive-dimensional weakly special subvarieties contained in $Y_{1}$ is not Zariski dense in $Y_{1}$, then take $N=N_{1}$. Otherwise by the same argument, there exists a normal subgroup $N_{1,2}$ of $P_{1}$ s.t. $\operatorname{dim}\left(N_{1,2}\right)>0$ and $\cup_{Z \in \mathfrak{F}\left(N_{1,2}, Y_{1}\right)} Z$ is Zariski dense in $Y_{1}$. Let $N_{2}:=\rho_{1}^{-1}\left(N_{1,2}\right)$, then $N_{2} \triangleleft P$. Draw the same diagram (12.6) with $N_{2}$ instead of $N_{1}$, then we get a mixed Shimura variety $S_{2}$ with $\operatorname{dim}\left(S_{2}\right)<\operatorname{dim}\left(S_{1}\right)$ and a Hodge generic subvariety $Y_{2}$ of $S_{2}$. Continue the process (if the union of positive-dimensional weakly special subvarieties contained in $Y_{2}$ is Zariski dense in $Y_{2}$ ).

Since $\operatorname{dim}(S)<\infty$, this process will end in a finite step. Hence there exists a number $k>0$ s.t. the union of positive-dimensional weakly special subvarieties contained in $Y_{k}$ is not Zariski dense in $Y_{k}$. Then $N:=N_{k}$ is the dezired subgroup of $P$.

\section{From Ax-Lindemann-Weierstrass to André-Oort}

For pure Shimura varieties, Ullmo and Pila-Tsimerman have explained separately in [61, §5] $[49, \S 7]$ how to deduce the André-Oort conjecture from the Ax-Lindemann-Weierstraß theorem with a suitable lower bound for Galois orbits of special points. In this section we first prove that in order to get a suitable lower bound for Galois orbits of special points for a mixed Shimura variety, it is enough to have one for its pure part. Then we show that the idea of Ullmo also works for mixed Shimura varieties.

13.1. Lower bounds for Galois orbits of special points. In this subsection, we will consider mixed Shimura data (resp. varieties) instead of only connected ones. See Definition 2.1.

Let $(P, \mathcal{X})$ be a mixed Shimura datum. Let $\pi:(P, \mathcal{X}) \rightarrow\left(G, \mathcal{X}_{G}\right)$ be the projection to its pure part. We use the notation of $\S 2.2$. In particular, we fix a Levi decomposition $P=W \rtimes G$ and an embedding $\left(G, \mathcal{X}_{G}\right) \hookrightarrow(P, \mathcal{X})$ as in $[67$, pp 6].

Let $K$ be an open compact subgroup of $P\left(\mathbb{A}_{f}\right)$ defined as follows: for $M>3$ even, $K_{U}:=$ $M U(\widehat{\mathbb{Z}}), K_{V}:=M V(\widehat{\mathbb{Z}}), K_{W}:=K_{U} \times K_{V}$ with the group law as in $\S 2.2, K_{G}:=\{g \in G(\widehat{\mathbb{Z}}) \mid g \equiv$ $1 \bmod M\}$ and $K:=K_{W} \rtimes K_{G}$. 
Let $s$ be a special point of $M_{K}(P, \mathcal{X})$ which corresponds to a special point $x \in \mathcal{X}$. The group $\operatorname{MT}(x)$ is of the form $w T w^{-1}$ for a torus $T \subset G$ and $w \in W(\mathbb{Q})$. Let $\operatorname{ord}(w) \in \mathbb{Z}_{>0}$ be the smallest integer such that $\operatorname{ord}(w) w \in W(\mathbb{Z})$. Define the order of $s$ to be $N(s):=\operatorname{ord}(w)$.

Remark 13.1. It is not hard to show that if the fiber of $S \stackrel{[\pi]}{\longrightarrow} S_{G}$ is a semi-abelian variety, then $N(s)$ coincides with the order of $s$ as a torsion point on the fiber (up to a constant).

Attached to $(P, \mathcal{X})$ there is a number field $E=E(P, \mathcal{X})$ called the reflex field and $M_{K}(P, \mathcal{X})$ is defined over $E$ (cf. $[53,11.5])$. We want a comparison of $|\operatorname{Gal}(\overline{\mathbb{Q}} / E) s|$ and $|\operatorname{Gal}(\overline{\mathbb{Q}} / E)[\pi](s)|$.

Define $\left(G^{w}, \mathcal{X}_{G^{w}}\right):=\left(w G w^{-1}, w^{-1} \cdot \mathcal{X}_{G}\right), K_{G^{w}}:=G^{w}\left(\mathbb{A}_{f}\right) \cap K$ and $K_{G}^{\prime}:=w^{-1} K_{G^{w}} w$, then we have the following commutative diagram:

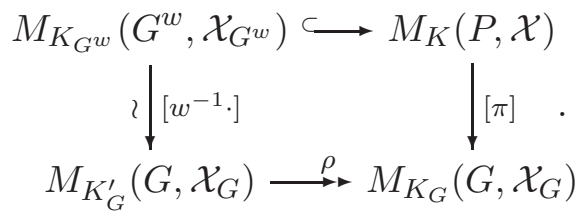

All the morphisms in this diagram are defined over $E$ since the reflex field of $(P, \mathcal{X}),\left(G, \mathcal{X}_{G}\right)$ and $\left(G^{w}, \mathcal{X}_{G^{w}}\right)$ are all $E$. Denote by $s^{\prime}:=\left[w^{-1}\right] \cdot s$. Let $T^{w}:=w T w^{-1}$. Let $K_{T}^{\prime}:=K \cap T^{w}\left(\mathbb{A}_{f}\right)$ and let $K_{T}:=K \cap T\left(\mathbb{A}_{f}\right)$. The following inequality follows essentially from [63, $\left.\S 2.2\right]$ (note that we do not need GRH for this inequality since [63, Lemma 2.13, 2.14] are not used!). We refer to $[28$, Theorem $1(1)]$ for a more precise version.

$|\operatorname{Gal}(\overline{\mathbb{Q}} / E) s|=\left|\operatorname{Gal}(\overline{\mathbb{Q}} / E) s^{\prime}\right| \geqslant B^{i(T)}\left|K_{T} / K_{T}^{\prime}\right|\left|\operatorname{Gal}(\overline{\mathbb{Q}} / E) \rho\left(s^{\prime}\right)\right|=B^{i(T)}\left|K_{T} / K_{T}^{\prime}\right||\operatorname{Gal}(\overline{\mathbb{Q}} / E)[\pi](s)|$ for some $B \in(0,1)$ depending only on $(P, \mathcal{X})$.

Write $w=(u, v)$ under the identification $W \simeq U \times V$ in $\S 2.2$. All elements of $w^{-1} K w$ are of the form

$$
(-u,-v, 1)\left(u^{\prime}, v^{\prime}, g^{\prime}\right)(u, v, 1)=\left(u^{\prime}-\left(u-g^{\prime} u\right)-\Psi\left(v, v^{\prime}\right), v^{\prime}-\left(v-g^{\prime} v\right), g^{\prime}\right)
$$

with $\left(u^{\prime}, v^{\prime}, g^{\prime}\right) \in K$. Since $K_{T}^{\prime}=w^{-1} K_{T w} w=w^{-1} K w \cap T\left(\mathbb{A}_{f}\right)$, this element is in $K_{T}^{\prime}$ iff

- $u^{\prime}=u-g^{\prime} u+\Psi\left(v, v^{\prime}\right) \in K_{U}$

- $v^{\prime}=v-g^{\prime} v \in K_{V}$

- $g^{\prime} \in T\left(\mathbb{A}_{f}\right) \cap K_{G}=K_{T}$.

So

$$
\begin{aligned}
& t \in K_{T} ; \\
& t \in w^{-1} K_{T^{w}} w \Longleftrightarrow v-t v \in K_{V}=M V(\widehat{\mathbb{Z}}) ; \\
& u-t u+\Psi(v, v-t v) \in K_{U}=M U(\widehat{\mathbb{Z}}) .
\end{aligned}
$$

Lemma 13.2. $\left|K_{T} / K_{T}^{\prime}\right| \geqslant \operatorname{ord}(w) \prod_{p \mid \operatorname{ord}(w)}\left(1-\frac{1}{p}\right)$.

Proof. Let $T^{\prime}$ be the image of $\mathbb{G}_{m, \mathbb{R}} \stackrel{\omega}{\rightarrow} \mathbb{S} \stackrel{w^{-1} \cdot x}{\longrightarrow} G_{\mathbb{R}}$, then it is an algebraic torus defined over $\mathbb{Q}$ by Remark 2.2(1). We always have $T^{\prime}<T$. If $T^{\prime}$ is trivial, then $P=G$ is adjoint by reason of weight, and $\operatorname{ord}(w)=1$. If not, $T^{\prime} \simeq \mathbb{G}_{m, \mathbb{Q}}$ and

$$
T^{\prime}(M):=\left\{t^{\prime} \in T^{\prime}(\widehat{\mathbb{Z}}) \mid t^{\prime} \equiv 1 \bmod (M)\right\} \subset K_{G} \cap T\left(\mathbb{A}_{f}\right)=K_{T} .
$$


So

$$
T^{\prime}(M) /\left(T^{\prime}(M) \cap w^{-1} K_{T^{w}} w\right) \hookrightarrow K_{T} / w^{-1} K_{T^{w}} w .
$$

Hence it is enough to prove that LHS is of cardinality $\geqslant \operatorname{ord}(w)$.

Since $T^{\prime}$ acts on $V$ and $U$ via a scalar, $t^{\prime} \in T^{\prime}(M) \cap w^{-1} K_{T^{w}} w$ iff

(1) $t^{\prime} \in T^{\prime}(M)$

(2) $v-t^{\prime} v \in M V(\widehat{\mathbb{Z}})$

(3) $u-t^{\prime} u \in M U(\widehat{\mathbb{Z}})$.

Let $t^{\prime} \in T^{\prime}(M) \subset T^{\prime}(\widehat{\mathbb{Z}})=\widehat{\mathbb{Z}}^{*}$. Suppose ord $(w)=\prod p^{n_{p}}$ and $M=\prod p^{m_{p}}$. If $n_{p}=0$, then condition (2) and (3) are automatically satisfied. If $n_{p}>0$, then condition (2) and (3) imply that $t_{p}^{\prime}=1+a_{n_{p}+m_{p}} p^{n_{p}+m_{p}}+\ldots \in \mathbb{Z}_{p}^{*}$, hence

$$
\left|T^{\prime}\left(\mathbb{Z}_{p}\right) \cap T^{\prime}(M) /\left(T^{\prime}\left(\mathbb{Z}_{p}\right) \cap T^{\prime}(M) \cap w^{-1} K_{T^{w}, p} w\right)\right|=p^{n_{p}-1}(p-1) .
$$

To sum up,

$$
\left|T^{\prime}(M) /\left(T^{\prime}(M) \cap w^{-1} K_{T^{w}} w\right)\right|=\operatorname{ord}(w) \prod_{p \mid \operatorname{ord}(w)}\left(1-\frac{1}{p}\right) .
$$

Theorem 13.3. For any $\varepsilon \in(0,1)$, there exist a positive constant $C_{\varepsilon}$ (depending only on $(P, \mathcal{X})$ and $\varepsilon$ ) such that

$$
|\operatorname{Gal}(\overline{\mathbb{Q}} / E) s| \geqslant C_{\varepsilon} N(s)^{1-\varepsilon}|\operatorname{Gal}(\overline{\mathbb{Q}} / E)[\pi](s)| .
$$

Proof. We have proved in Lemma 13.2

$$
p \mid \operatorname{ord}(w) \Longleftrightarrow K_{T, p} \neq K_{T, p}^{\prime} .
$$

Hence denoting by $\varsigma(M):=|\{p, p \mid M\}|$ for any $M \in \mathbb{Z}_{>0}$, we have by Lemma 13.2 and (13.1)

$$
|\operatorname{Gal}(\overline{\mathbb{Q}} / E) s| \geqslant B^{\varsigma(N(s))} N(s) \prod_{p \mid N(s)}\left(1-\frac{1}{p}\right)\left|\operatorname{Gal}(\overline{\mathbb{Q}} / E) \rho\left(s^{\prime}\right)\right| .
$$

Now the theorem follows from the basic facts of elementary math:

$$
\begin{gathered}
\forall \varepsilon \in(0,1) \text {, there exists } C_{\varepsilon}>0 \text { such that } B^{\varsigma(N(s))} N(s)^{\varepsilon} \geqslant C_{\varepsilon} . \\
\forall \varepsilon \in(0,1), \text { there exists } C_{\varepsilon}^{\prime}>0 \text { such that } N(s)^{\varepsilon} \prod_{p \mid N(s)}\left(1-\frac{1}{p}\right) \geqslant C_{\varepsilon}^{\prime} .
\end{gathered}
$$

Corollary 13.4. For $A$ an abelian variety over a number field $k \subset \mathbb{C}$ and $t$ a torsion point of $A(\mathbb{C})$, denote by $N(t)$ its order and $k(t)$ the field of definition of $t$ over $k$.

Let $g \in \mathbb{N}_{+}$and let $\varepsilon \in(0,1)$. There exists $c>0$ such that for all number fields $k \subset \mathbb{C}$, all $g$-dimensional $C M$ abelian varieties $A$ with definition field $k$ and all torsion points $t$ in $A(\mathbb{C})$,

$$
[k(t): k] \geqslant c N(t)^{1-\varepsilon} \text {. }
$$

Proof. (compare with [57]) By Zarhin's trick, it suffices to give a proof for $A$ principally polarized. Such an $A$ can be realized as a fiber of $\mathfrak{A}_{g}(4) \rightarrow \mathcal{A}_{g}(4)$, and any torsion point $t$ of $A$ is a special point of $\mathfrak{A}_{g}(4)$. Now this result is a direct consequence of Proposition 13.3. 
Remark 13.5. The lower bound of the Galois orbit of a special point for pure Shimura varieties is given by [61, Conjecture 2.7]. It has been proved under the Generalized Riemann Hypothesis by Ullmo-Yafaev [63]. For the case of $\mathcal{A}_{g}$, it is equivalent to the following conjectural lower bound (suggested and proved for $g=2$ by Edixhoven [26, 25]): suppose that $x \in \mathcal{A}_{g}$ is a special point. Let $A_{x}$ denote the $C M$ abelian variety parametrised by $x$ and let $R_{x}$ be the center of $\operatorname{End}\left(A_{x}\right)$, then there exists $\delta(g)>0$ such that

$$
|\operatorname{Gal}(\overline{\mathbb{Q}} / \mathbb{Q}) x| \gg_{g}\left|\operatorname{disc}\left(R_{x}\right)\right|^{\delta(g)} .
$$

For their equivalence see [59, Theorem 7.1]. The best unconditional result is given by Tsimerman [59, Theorem 1.1]: (13.8) is true when $g \leqslant 6$ (and for $g \leqslant 3$ by a similar method in [65]).

Hence for a mixed Shimura variety of Siegel type of genus $g$ and any special point $x$, Theorem 13.3 tells us that if [61, Conjecture 2.7] is verified for the pure part, then for any $\varepsilon \in(0,1)$, there exists $\delta(g)>0$ such that

$$
|\operatorname{Gal}(\overline{\mathbb{Q}} / \mathbb{Q}) x| \gg_{g, \varepsilon} N(x)^{1-\varepsilon}\left|\operatorname{disc}\left(R_{[\pi](x)}\right)\right|^{\delta(g)} .
$$

\subsection{André-Oort.}

Theorem 13.6. Let $S$ be a connected mixed Shimura variety of abelian type (i.e. its pure part is of abelian type). Let $Y$ be a closed irreducible subvariety of $S$ containing a Zariski-dense set of special points. If (13.8) holds for the pure part of $S$, then $Y$ is special.

In particular, by the main result of [59], the André-Oort conjecture holds unconditionally for any mixed Shimura variety whose pure part is a subvariety of $\mathcal{A}_{6}^{n}$.

Proof. Suppose $S$ is associated with $\left(P, \mathcal{X}^{+}\right)$. Replacing $\Gamma$ by a neat subgroup does not change the assumption or the conclusion, so we may assume that $\Gamma=\{\gamma \in P(\mathbb{Z}) \mid \gamma \equiv 1 \bmod N\}$ for some $N>3$ even. Replacing $S$ by the smallest connected mixed Shimura subvariety does not change the assumption or the conclusion, so we may assume that $Y$ is Hodge generic in $S$. Since $Y$ contains a Zariski-dense set of special points, we may assume that $Y$ is defined over a number field $k$. Suppose that $Y$ is not special.

If the set of positive-dimensional weakly special subvarieties of $Y$ is Zariski dense in $Y$, then let $N$ be the normal subgroup $P$ as in Theorem 12.2. Consider the diagram (12.1), then $Y$ is special iff $Y^{\prime}:=\overline{[\rho](Y)}$ is. It is clear that $S^{\prime}$ is again of abelian type. Replacing $(S, Y)$ by $\left(S^{\prime}, Y^{\prime}\right)$, we may assume that the set of positive-dimensional special subvarieties of $Y$ is not Zariski dense in $Y$.

Now we are left prove that the set of special points of $Y$ which do not lie in any positivedimensional special subvariety is finite.

By definition, there exists a Shimura morphism $\left(G, \mathcal{X}_{G}^{+}\right) \rightarrow \prod_{i=1}^{r}\left(\mathrm{GSp}_{2 g}^{(i)}, \mathbb{H}_{g}^{+(i)}\right)$ (the upperindex $(i)$ is to distinguish different factors) s.t. $G \rightarrow \prod_{i=1}^{r} \mathrm{GSp}_{2 g}^{(i)}$ has a finite kernel (contained in the center) and $\mathcal{X}_{G}^{+} \hookrightarrow \prod_{i=1}^{r} \mathbb{H}_{g}^{+(i)}$. Therefore under Proposition 4.3 , we can identify $\mathcal{X}^{+}$as a subspace of $U(\mathbb{C}) \times V(\mathbb{R}) \times \mathbb{H}_{g}^{+r}$. Then any special point in contained in $U(\mathbb{Q}) \times V(\mathbb{Q}) \times\left(\mathbb{H}_{g}^{+r} \cap\right.$ $M_{2 g}(\overline{\mathbb{Q}})^{r}$ ) and hence we can define its height (for $\overline{\mathbb{Q}}$-points, see [13, Definition 1.5.4 multiplicative height]).

Now take $\mathcal{F}$ as in $\S 10.1$. For any special point $x \in S$, take a representative $\widetilde{x} \in$ unif $^{-1}(x)$ in $\mathcal{F}$, then by [50, Theorem 3.1], $H\left(\widetilde{x}_{G, i}\right) \ll\left|\operatorname{disc}\left(R_{[\pi](x)_{i}}\right)\right|^{B_{g}}$ for a constant $B_{g}(\forall i=1, \ldots, r)$. By 
choice of $\mathcal{F}, H\left(\widetilde{x}_{V}\right), H\left(\widetilde{x}_{U}\right) \ll N(x)$ (see Remark 4.4). If (13.8) holds, then by Proposition 13.3

$$
\#(\operatorname{Gal}(\overline{\mathbb{Q}} / k) x) \gg_{g} H(\widetilde{x})^{\varepsilon(g)}
$$

for some $\varepsilon(g)>0$. Hence for $H(\widetilde{x}) \gg 0$, Pila-Wilkie $[47,3.2]$ implies that $\exists \sigma \in \mathrm{Gal}(\overline{\mathbb{Q}} / k)$ s.t. $\widetilde{\sigma(x)}$ is contained in a semi-algebraic subset of unif $^{-1}(Y) \cap \mathcal{F}$ of positive dimension. But by [61, pp6, last line],

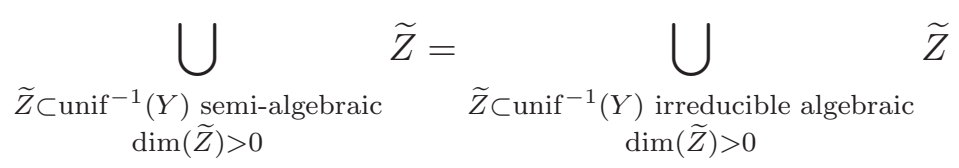

So $\widetilde{\sigma(x)}$ is contained in some maximal algebraic subset $\widetilde{Z}$ of unif $^{-1}(Y)$ of positive dimension. Theorem 1.2 tells us that $\widetilde{Z}$ is weakly special. Hence $\sigma^{-1}(Z)(Z:=\operatorname{unif}(\widetilde{Z}))$ is weakly special containing a special point $x$. Hence $\sigma^{-1}(Z)$ is special of positive dimension. To sum up, the heights of the elements of

$\left\{\widetilde{x} \in\right.$ unif $^{-1}(Y) \cap \mathcal{F}$ special and unif $(\widetilde{x})$ is not contained in a positive-dimensional special subvariety $\}$ is uniformly bounded, and hence this set is finite by Northcott's theorem [13, Theorem 1.6.8].

\section{Appendix}

We prove here Theorem 7.6 when $E=T$ is an algebraic torus over $\mathbb{C}$ (which corresponds to the case $W=U$ ) and when $E=A$ is a complex abelian variety (which corresponds to the case $W=V$ ). The proof is a rearrangement of existing proofs (combine the point counting of Pila-Zannier [52] and volume calculation of Ullmo-Yafaev [64]). Use notation in $\S 11$.

Case $i: E=A$. In this case, $W=V$ and $\Gamma_{V}=\oplus_{i=1}^{2 n} \mathbb{Z} e_{i} \subset \operatorname{Lie}(A)=\mathbb{C}^{n}=\mathbb{R}^{2 n}$ is a lattice. Denote by univ: $\operatorname{Lie}(A) \rightarrow A$. Let $\mathcal{F}_{V}:=\Sigma_{i=1}^{2 n}(-1,1) e_{i}$, then $\mathcal{F}_{V}$ is a fundamental set for the action of $\Gamma_{V}$ on $\operatorname{Lie}(A)$ s.t. $\left.u n i v\right|_{\mathcal{F}_{V}}$ is definable. Define the norm of $z=\left(x_{1}, y_{1}, \ldots, x_{n}, y_{n}\right) \in$ $\operatorname{Lie}(A)=\mathbb{R}^{2 n}$ to be

$$
\|z\|:=\operatorname{Max}\left(\left|x_{1}\right|,\left|y_{1}\right|, \ldots,\left|x_{n}\right|,\left|y_{n}\right|\right) .
$$

It is clear that $\forall z \in \operatorname{Lie}(A)$ and $\forall \gamma_{V} \in \Gamma_{V}$ s.t. $\gamma_{V} z \in \mathcal{F}_{V}$,

$$
H\left(\gamma_{V}\right) \ll\left\|x_{V}\right\| \text {. }
$$

Let $\omega_{V}:=d z_{1} \wedge d \bar{z}_{1}+\ldots+d z_{n} \wedge d \bar{z}_{n}$ be the canonical $(1,1)$-form of $\operatorname{Lie}(A)=\mathbb{C}^{n}$. Let $p_{i}$ $(i=1, \ldots, n)$ be the $n$ natural projections of $\operatorname{Lie}(A)=\mathbb{C}^{n}$ to $\mathbb{C}$. Let $C$ be an algebraic curve of $\widetilde{Z}$ and define $C_{M}:=\{z \in C \mid\|z\| \leqslant M\}$. We have

$$
\begin{aligned}
\int_{C \cap \mathcal{F}_{V}} \omega_{V} & \leqslant d \sum_{i=1}^{n} \int_{p_{i}\left(C \cap \mathcal{F}_{V}\right)} d z_{i} \wedge d \bar{z}_{i} \\
& \leqslant d \sum_{i=1}^{n} \int_{p_{i}\left(\mathcal{F}_{V}\right)} d z_{i} \wedge d \bar{z}_{i}=d \cdot O(1)
\end{aligned}
$$

and

$$
\int_{C_{M}} \omega_{V} \geqslant O\left(M^{2}\right)
$$

with $d=\operatorname{deg}(C)$ by [33, Theorem 0.1]. 
By (14.1)

$$
C_{M} \subset \bigcup_{\gamma_{V} \in \Theta(\widetilde{Z}, M)}\left(C \cap \gamma^{-1} \mathcal{F}\right) .
$$

Integrating both side w.r.t. $\omega_{V}$ we have

$$
M^{2} \ll \# \Theta(\widetilde{Z}, M)
$$

by (14.2) and (14.3).

Let $\operatorname{Stab}_{V}(\widetilde{Z}):={\overline{\Gamma_{V} \cap \operatorname{Stab}_{V(\mathbb{R})}(\widetilde{Z})}}^{\text {Zar }}$. Now by Pila-Wilkie [64, Theorem 3.4], the exists an semi-algebraic block $B \subset \Sigma(\widetilde{Z})$ of positive dimension containing arbtrarily many points $\gamma_{V} \in \Gamma_{V}$. We have $B \widetilde{Z} \subset u_{n i v}^{-1}(Y)$ since $\Sigma(\widetilde{Z}) \widetilde{Z} \subset u_{n i v}^{-1}(Y)$ by definition. Hence for any $\gamma_{V} \in \Gamma_{V} \cap B, \widetilde{Z} \subset \gamma_{V}^{-1} B \widetilde{Z} \subset$ univ $^{-1}(Y)$, and therefore $\widetilde{Z}=\gamma_{V}^{-1} B \widetilde{Z}$ by maximality of $\widetilde{Z}$. So $\gamma_{V}^{-1}\left(B \cap \Gamma_{V}\right) \subset \operatorname{Stab}_{V}(\widetilde{Z})(\mathbb{Q})$, and hence $\operatorname{dim}\left(\operatorname{Stab}_{V}(\widetilde{Z})\right)>0$. For any point $\widetilde{z} \in \widetilde{Z}$, $\operatorname{Stab}_{V}(\widetilde{Z})(\mathbb{R})+\widetilde{z} \subset \widetilde{Z}$. By [52, Lemma 2.3], $\operatorname{Stab}_{V}(\widetilde{Z})(\mathbb{R})$ is full and complex. Define $V^{\prime}:=$ $V / \operatorname{Stab}_{V}(\widetilde{Z})$ and $\Gamma_{V^{\prime}}:=\Gamma_{V} /\left(\Gamma_{V} \cap \operatorname{Stab}_{V}(\widetilde{Z})(\mathbb{Q})\right)$, and then $A^{\prime}:=V^{\prime}(\mathbb{R}) / \Gamma_{V^{\prime}}$ is a quotient abelian variety of $A$. Let $Y^{\prime}$ (resp. $\widetilde{Z}^{\prime}$ ) be the Zariski closure of the projection of $Y$ (resp. $\widetilde{Z}$ ) in $A^{\prime}$ (resp. $\left.V^{\prime}(\mathbb{R})\right)$. We prove that the image of $\widetilde{Z}^{\prime}$ is a point. If not, then proceeding as before for the triple $\left(A^{\prime}, Y^{\prime}, \widetilde{Z}^{\prime}\right)$ can we prove $\operatorname{dim}\left(\operatorname{Stab}_{V^{\prime}}\left(\widetilde{Z}^{\prime}\right)\right)>0$. This contradicts to the definition (maximality) of $\operatorname{Stab}_{V}(\widetilde{Z})$. Hence $\widetilde{Z}$ is a translate of $\operatorname{Stab}_{V}(\widetilde{Z})(\mathbb{R})$. So $\widetilde{Z}$ is weakly special.

Case ii : $E=T$. Define the norm of $x_{U}=\left(x_{U, 1}, x_{U, 2}, \ldots, x_{U, m}\right) \in U(\mathbb{C})$ to be

$$
\left\|x_{U}\right\|:=\operatorname{Max}\left(\left\|x_{U, 1}\right\|,\left\|x_{U, 2}\right\|, \ldots,\left\|x_{U, m}\right\|\right) .
$$

It is clear that $\forall x_{U} \in U(\mathbb{C})$ and $\forall \gamma_{U} \in \Gamma_{U}$ s.t. $\gamma_{U} x_{U} \in \mathcal{F}_{U}$,

$$
H\left(\gamma_{U}\right) \ll\left\|x_{U}\right\| \text {. }
$$

Let $\left.\omega\right|_{T}=d z_{1} \wedge d \bar{z}_{1}+\ldots+d z_{m} \wedge d \bar{z}_{m}$ be the canonical $(1,1)$-form of $U(\mathbb{C}) \simeq \mathbb{C}^{m}$. Let $p_{i}$ $(i=1, \ldots, m)$ be the $m$ natural projections of $U(\mathbb{C}) \simeq \mathbb{C}^{m}$ to $\mathbb{C}$. Let $C$ be an algebraic curve of $\widetilde{Z}$ and define $C_{M}:=\{x \in C \mid\|x\| \leqslant M\}$. We have

$$
\begin{aligned}
\left.\int_{C_{M} \cap \mathcal{F}_{U}} \omega\right|_{T} & \leqslant d \sum_{i=1}^{m} \int_{p_{i}\left(C_{M} \cap \mathcal{F}_{U}\right)} d z_{i} \wedge d \bar{z}_{i} \\
& \leqslant d \sum_{i=1}^{m} \int_{\{s \in \mathbb{C} \mid-1<\Re \mathfrak{R}(s)<1,\|s\| \leqslant M\}} d z_{i} \wedge d \bar{z}_{i}=d \cdot O(M)
\end{aligned}
$$

where $d:=\operatorname{deg}(C)$. On the other hand by [33, Theorem 0.1],

$$
\left.\int_{C_{M}} \omega\right|_{T} \geqslant O\left(M^{2}\right)
$$

By (14.4)

$$
C_{M} \subset \bigcup_{\gamma \in \Theta(\widetilde{Z}, M)}\left(C_{M} \cap \gamma^{-1} \mathcal{F}\right) .
$$

Integrating both side w.r.t. $\left.\omega\right|_{T}$ and taking into account that

$$
\gamma \cdot C_{M} \subset(\gamma C)_{2 M} \quad \text { if } H(\gamma) \leqslant M,
$$


we have

$$
M^{2} \ll \# \Theta(\widetilde{Z}, M) \cdot M
$$

by $(14.5)$ and $(14.6)$. Hence $\# \Theta(\widetilde{Z}, M) \gg M$.

Let $\operatorname{Stab}_{U}(\widetilde{Z}):={\overline{\Gamma_{U} \cap \operatorname{Stab}_{U(\mathbb{C})}(\widetilde{Z})}}^{\text {Zar }}$. Now by Pila-Wilkie [47, Theorem 3.6], the exists an semi-algebraic subset $B \subset \Sigma(\widetilde{Z})$ of positive dimension containing arbtrarily many points $\gamma_{U} \in \Gamma_{U}$. We have $B \widetilde{Z} \subset$ univ $^{-1}(Y)$ since $\Sigma(\widetilde{Z}) \widetilde{Z} \subset u_{n i v}^{-1}(Y)$ by definition. Hence for any $\gamma_{U} \in \Gamma_{U} \cap B, \widetilde{Z} \subset \gamma_{U}^{-1} B \widetilde{Z} \subset$ univ $^{-1}(Y)$, and therefore $\widetilde{Z}=\gamma_{U}^{-1} B \widetilde{Z}$ by maximality of $\widetilde{Z}$. So $\gamma_{U}^{-1}\left(B \cap \Gamma_{U}\right) \subset \operatorname{Stab}_{U}(\widetilde{Z})(\mathbb{Q})$, and hence $\operatorname{dim}\left(\operatorname{Stab}_{U}(\widetilde{Z})\right)>0$. Let $U^{\prime}:=U / \operatorname{Stab}_{U}(\widetilde{Z})$, $\Gamma_{U^{\prime}}:=\Gamma_{U} /\left(\Gamma_{U} \cap \operatorname{Stab}_{U}(\widetilde{Z})(\mathbb{Q})\right)$ and $T^{\prime}:=U^{\prime}(\mathbb{C}) / \Gamma_{U^{\prime}} . T^{\prime}$ is an algebraic torus over $\mathbb{C}$. Let $Y^{\prime}$ (resp. $\widetilde{Z}^{\prime}$ ) be the Zariski closure of the projection of $Y$ (resp. $\left.\widetilde{Z}\right)$ in $T^{\prime}\left(\operatorname{resp} . U^{\prime}(\mathbb{C})\right.$ ). We prove that $\widetilde{Z}^{\prime}$ is a point. If not, then proceeding as before for the triple $\left(T^{\prime}, Y^{\prime}, \widetilde{Z}^{\prime}\right)$ we can prove $\operatorname{dim}\left(\operatorname{Stab}_{U^{\prime}}\left(\widetilde{Z}^{\prime}\right)\right)>0$. This contradicts the definition (maximality) of $\operatorname{Stab}_{U}(\widetilde{Z})$. Hence $\widetilde{Z}$ is a translate of $\operatorname{Stab}_{U}(\widetilde{Z})(\mathbb{C})$. So $\widetilde{Z}$ is weakly special.

\section{REFERENCES}

[1] Y. André. Mumford-Tate groups of mixed Hodge structures and the theorem of the fixed part. Compositio Mathematica, 82(1):1-24, 1992.

[2] Y. André. Finitude des couples d'invariants modulaires singuliers sur une courbe algébrique plane non modulaire. J.Reine Angew. Math (Crelle), 505:203-208, 1998.

[3] Y. André. Shimura varieties, subvarieties, and CM points. Six lectures at the University of Hsinchu, AugustSeptember 2001.

[4] A. Ash, D. Mumford, D. Rapoport, and Y. Tai. Smooth compactifications of locally symmetric varieties (2nd edition). Cambridge Mathematical Library. Cambridge University Press, 2010.

[5] J. Ax. On Schanuel's conjectures. Annals Math., 93:252-268, 1971.

[6] J. Ax. Some topics in differential algebraic geometry I: Analytic subgroups of algebraic groups. American Journal of Mathematics, 94:1195-1204, 1972.

[7] W. Baily and A. Borel. Compactification of arithmetic quotients of bounded symmetric domains. Annals Math., 84(3):442-528, 1966.

[8] D. Bertrand. Special points and Poincaré bi-extensions. Preprint, available on the author's page. with an appendix by B.Edixhoven.

[9] D. Bertrand. Unlikely intersections in Poincaré biextensions over elliptic schemes. Notre Dame J. Formal Logic, 54(3-4):365-375, 2013.

[10] D. Bertrand and B. Edixhoven. Pink's conjecture, Poincaré bi-extensions and generalized Jacobians. in preparation.

[11] D. Bertrand, D. Masser, A. Pillay, and U. Zannier. Relative Manin-Mumford for semi-abelian surfaces. Preprint, available on the authors' page.

[12] D. Bertrand and A. Pillay. A Lindemann-Weierstrass theorem for semi-abelian varieties over function fields. J.Amer.Math.Soc., 23(2):491-533, 2010.

[13] E. Bombieri and W. Gubler. Heights in diophantine geometry. Camb. Univ. Press, 2006.

[14] A. Borel. Linear Algebraic Groups, volume 126 of GTM. Springer, 1991.

[15] A. Chambert-Loir. Relations de dépendance et intersections exceptionnelles. Séminaire Bourbaki, exposé n. 1032, 63e année, 2010-2011.

[16] L. Clozel and E. Ullmo. Équidistribution de sous-variétés spéciales. Annals Math., 161:1571-1588, 2005.

[17] L. Clozel and E. Ullmo. Équidistribution adélique des tores et équidistribution des points CM. Doc. Math, en l'honneur de J.Coates:233-260., 2006.

[18] C. Daw. Degrees of strongly special subvarieties and the Andre-Oort conjecture J.Reine Angew. Math (Crelle), to appear. 
[19] C. Daw and A. Yafaev. An unconditional proof of the André-Oort conjecture for Hilbert modular surfaces. Manuscripta Mathematica, 135(1-2):263-271, May 2011.

[20] P. Deligne. Travaux de Shimura, volume 244 of LNM. Springer, 1971.

[21] P. Deligne. Variété de Shimura: interprétation modulaire et techniques de construction de modèles canoniques. In Automorphic forms, Shimura varieties, and L-functions. Part 2, volume 33 of Proc. of Symp. in Pure Math, pages 274-290. Amer. Math. Soc., 1979.

[22] P. Deligne, J. Milne, A. Ogus, and K. Shih. Hodge cycles, motives, and Shimura varieties, volume 900 of LNM. Springer-Verlag, 1982.

[23] L. van den Dries. Tame Topology and o-minimal Structures, volume 248 of London Math. Soc. Lecture Note Series. Camb. Univ. Press, 1998.

[24] B. Edixhoven. Special points on the product of two modular curves. Compositio Mathematica, 114(3):315-328, 1998.

[25] B. Edixhoven. On the André-Oort conjecture for Hilbert modular surfaces. In Moduli of abelian varieties (Texel Island, 1999), volume 195, pages 133-155. Birkhäuser, Basel, 2001.

[26] B. Edixhoven, B. Moonen, and F. Oort. Open problems in algebraic geometry. Bull. Sci. Math., 125:1-22, 2001.

[27] B. Edixhoven and A. Yafaev. Subvarieties of Shimura varieties. Annals Math., 157(2):621-645, 2003.

[28] Z. Gao. Comparison of Galois orbits of special points of Shimura varieties. Note, available on the authors' page.

[29] A. Grothendieck and J. Dieudonné. Éléments de géométrie algébrique (rédigés avec la collaboration de Jean Dieudonné): IV. Étude locale des schémas et des morphismes de schémas, Première partie, volume 20. Publications Mathématiques de l'IHÉS, 1964.

[30] P. Habegger. Special points on fibered powers of elliptic surfaces. J.Reine Angew. Math (Crelle), to appear.

[31] P. Habegger and J. Pila. Some unlikely intersections beyond André-Oort. Compositio Mathematica, 148(01):127, January 2012.

[32] R. Hain and S. Zucker. Unipotent variations of mixed Hodge structure. Inv. Math., 88:83-124, 1987.

[33] J. Hwang and W. To. Volumes of complex analytic subvarieties of Hermitian symmetric spaces. American Journal of Mathematics, 124(6):1221-1246, 2002.

[34] M. Kashiwara. A study of variation of mixed Hodge structure. Publ. RIMS Kyoto Univ., 22:991-1024, 1986.

[35] B. Klingler, E. Ullmo, and A. Yafaev. The hyperbolic Ax-Lindemann-Weierstrass conjecture. Preprint, available on the authors' page.

[36] B. Klingler and A. Yafaev. The André-Oort conjecture. Annals Math., to appear.

[37] J. Kollár. Shafarevich maps and automorphic forms. Princeton Univ. Press, 1995.

[38] J. Milne. Canonical models of (mixed) Shimura varieties and automorphic vector bundles. In Automorphic forms, Shimura varieties, and L-functions. Vol. I. Proceedings of the conference held at the University of Michigan, Ann Arbor, Michigan, July 6-16 1988.

[39] J. Milne. Introduction to Shimura varieties. In Harmonic analysis, the trace formula, and Shimura varieties, volume 4 of Clay Math. Proc. Amer. Math. Soc., 2005.

[40] B. Moonen. Linearity properties of Shimura varieties, I. Journal of Algebraic Geometry, 7(3):539-467, 1988.

[41] D. Mumford. The red book of varieties and schemes, Second, expanded edition, volume 1358 of LNM. Springer, 1999.

[42] M. Orr. La conjecture d'André-Pink: Orbites de Hecke et sous-variétés faiblement spéciales. PhD thesis, Université Paris-Sud, 2013.

[43] M. Orr. Families of abelian varieties with many isogenous fibres. J.Reine Angew. Math (Crelle), to appear.

[44] C. Peters and J. Steenbrink. Mixed Hodge Structures, volume 52 of A Series of Modern Surveys in Mathematics. Springer, 2008.

[45] Y. Peterzil and S. Starchenko. Definability of restricted theta functions and families of abelian varieties. Duke Journal of Mathematics, 162:731-765, 2013.

[46] Y. Peterzil and S. Starchenko. Around Pila-Zannier: the semi-abelian case. Available on the authors' page.

[47] J. Pila. O-minimality and the André-Oort conjecture for $\mathbb{C}^{n}$. Annals Math., 173:1779-1840, 2011.

[48] J. Pila. Special point problems with elliptic modular surfaces. Mathematika, to appear.

[49] J. Pila and J. Tsimerman. Ax-Lindemann for $\mathcal{A}_{g}$. Annals Math., to appear. 
[50] J. Pila and J. Tsimerman. The André-Oort conjecture for the moduli space of Abelian surfaces. Compositio Mathematica, 149:204-216, February 2013.

[51] J. Pila and A. Wilkie. The rational points of a definable set. Duke Journal of Mathematics, 133:591-616, 2006.

[52] J. Pila and U. Zannier. Rational points in periodic analytic sets and the Manin-Mumford conjecture. Rend. Mat. Acc. Lincei, 19:149-162, 2008.

[53] R. Pink. Arithmetical compactification of mixed Shimura varieties. PhD thesis, Bonner Mathematische Schriften, 1989.

[54] R. Pink. A combination of the conjectures of Mordell-Lang and André-Oort. In Geometric Methods in Algebra and Number Theory, volume 253 of Progress in Mathematics, pages 251-282. Birkhäuser, 2005.

[55] V. Platonov and A. Rapinchuk. Algebraic Groups and Number Theory. Academic Press, INC., 1994.

[56] G. Rémond. Autour de la conjecture de Zilber-Pink. Journal de Théorie des Nombres de Bordeaux, 21(2):405414, 2009.

[57] A. Silverberg. Torsion points on abelian varieties of CM-type. Compositio Mathematica, 68:241-249., 1988.

[58] J. Steenbrink and S. Zucker. Variation of mixed Hodge structure I. Inv. Math., 80:489-542, 1985.

[59] J. Tsimerman. Brauer-Siegel for arithmetic tori and lower bounds for Galois orbits of special points. J.Amer.Math.Soc., 25:1091-1117, 2012.

[60] E. Ullmo. Autour de la conjecture d'André-Oort. Available on the author's page. Notes de cours pour les états de la recherche sur la conjecture de Zilber-Pink (CIRM 2011).

[61] E. Ullmo. Quelques applications du théorème de Ax-Lindemann hyperbolique. Compositio Mathematica, to appear.

[62] E. Ullmo and A. Yafaev. A characterisation of special subvarieties. Mathematika, 57(2):263-273, 2011.

[63] E. Ullmo and A. Yafaev. Galois orbits and equidistribution of special subvarieties: towards the André-Oort conjecture. Annals Math., to appear.

[64] E. Ullmo and A. Yafaev. The hyperbolic Ax-Lindemann in the compact case. Duke Journal of Mathematics, to appear.

[65] E. Ullmo and A. Yafaev. Nombre de classes des tores de multiplication complexe et bornes inférieures pour orbites Galoisiennes de points spéciaux. Bull. de la $S M F$, to appear.

[66] C. Voisin. Hodge theory and complex algebraic geometry I, volume 76 of Cambridge Studies in Advanced Mathematics. Camb. Univ. Press, 2002.

[67] J. Wildeshaus. The canonical construction of mixed sheaves on mixed Shimura varieties. In Realizations of Polylogarithms, volume 1650 of LNM, pages 77-140. Springer, 1997.

[68] A. Yafaev. On a result of Ben Moonen on the moduli space of principally polarised abelian varieties. Compositio Mathematica, 141(05):1103-1108, 2005.

[69] A. Yafaev. A conjecture of Yves André's. Duke Journal of Mathematics, 132(3):393-407, 2006.

[70] B. Zilber. Exponential sums equations and the Schanuel conjecture. Journal of the London Mathematical Society, 65(01):27-44, February 2002.

Institut des Hautes Études Scientifiques, Le Bois-Marie 35, Route de Chartres, 91440 BuresSUR-YvetTE, FRANCE

E-mail address: ziyang.gao@math.u-psud.fr 